\title{
Functional Analysis of the Salicylic Acid-Responsive PR-1 Promoter in Arabidopsis thaliana
}

\author{
Dissertation \\ zur Erlangung des Doktorgrades \\ der Mathematisch-Naturwissenschaftlichen Fakultäten \\ der Georg-August-Universität zu Göttingen
}

\author{
vorgelegt von \\ Sebastian Pape \\ aus Brakel
}

Göttingen 2009 
D7

Referent:

Prof. Dr. Christiane Gatz

Korreferent:

PD Dr. Wolfgang Dröge-Laser

Tag der mündlichen Prüfung:

9. Juli 2009 



\section{Contents}

\section{Abbreviations}

1 Summary. ...Fehler! Textmarke nicht definiert.

2 Introduction 6

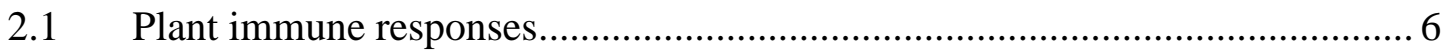

2.2 Plant hormones and their role in host defense …....................................... 7

2.3 SA signaling and systemic acquired resistance (SAR) ................................ 10

2.4 NPR1-, WRKY- and SNI1 dependent SA signaling .................................. 13

2.5 TGA transcription factors and their role in SAR ......................................... 16

2.6 The regulatory element activating sequence 1 (as-1) ................................... 18

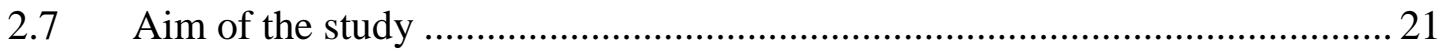

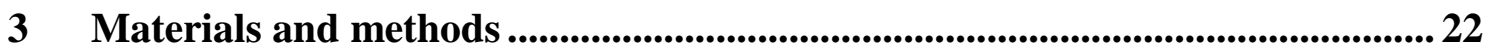

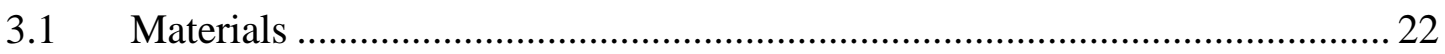

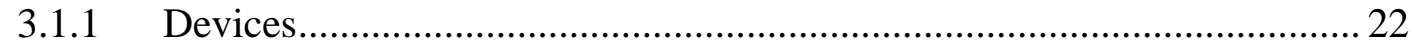

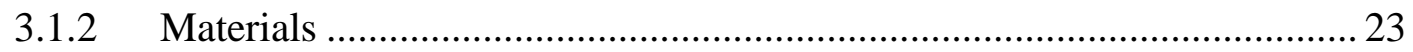

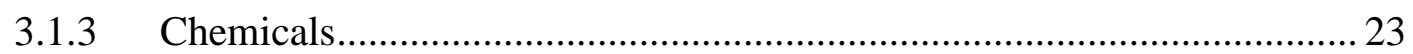

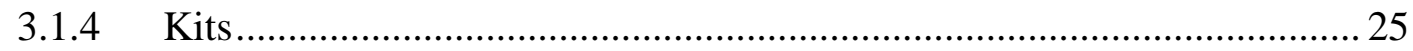

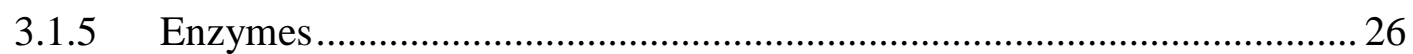

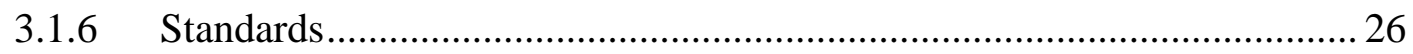

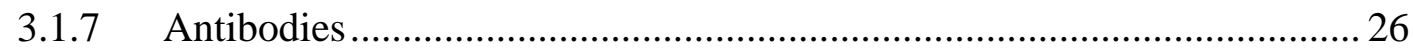

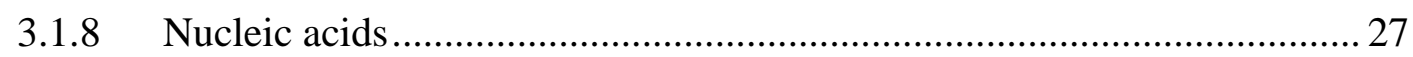

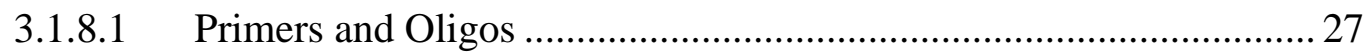

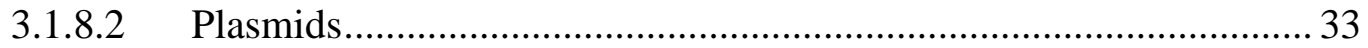

3.1.8.2.1 Plasmids for promoter analysis purposes ................................. 33

3.1.8.2.2 Plasmids for cloning- and transient assay purposes ..................... 36

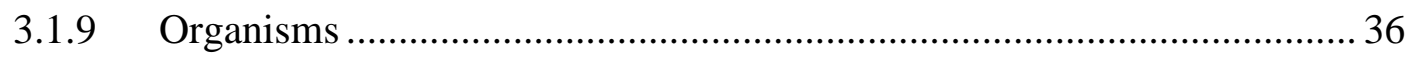

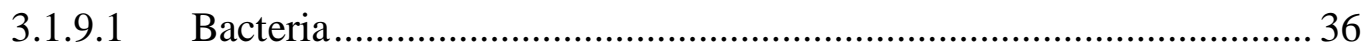

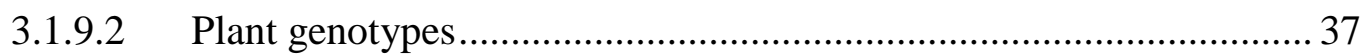

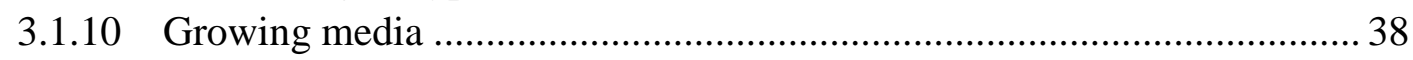

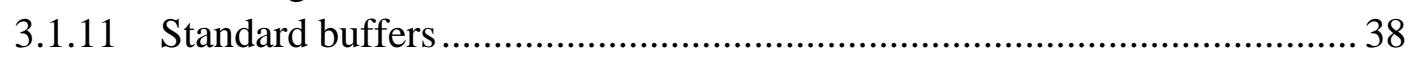

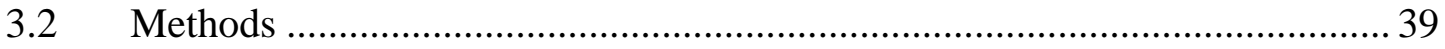

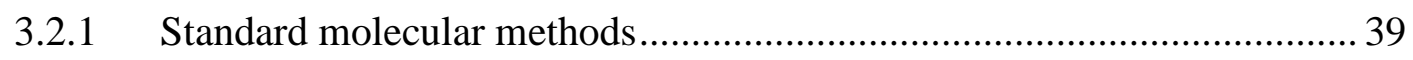

3.2.1.1 Isolation of plasmid DNA from E. coli ......................................... 39

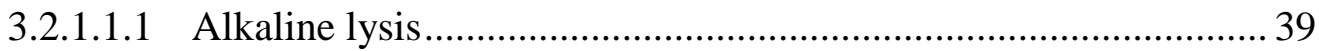

3.2.1.1.2 Isolation of high- quality plasmid DNA....................................... 39 
3.2.1.2 Measurement of DNA- and RNA concentrations ............................. 40

3.2.1.3 Separation of DNA on agarose gels ................................................ 40

3.2.1.4 Separation of DNA on polyacrylamid gels (PAA) .......................... 40

3.2.1.5 Restriction digestion of DNA …................................................ 41

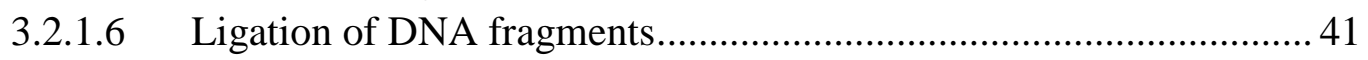

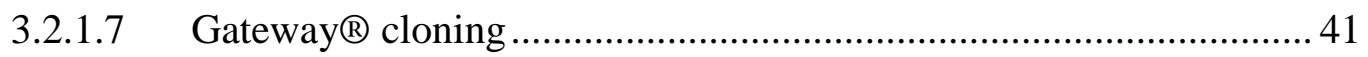

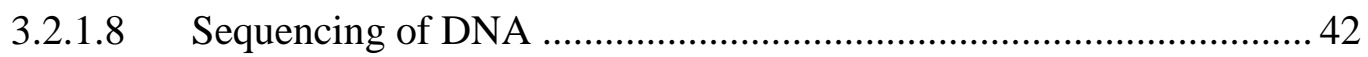

3.2.1.9 Cloning of vectors ................................................................... 42

3.2.1.9.1 Hybridization of complementary DNA fragments ....................... 42

3.2.1.9.2 Cloning of DNA fragments for EMSA studies ........................... 42

3.2.1.9.3 Cloning of full length $P R-1$ promoter constructs ........................ 43

3.2.1.9.4 Cloning of $P R-1$ promoter deletion constructs........................... 44

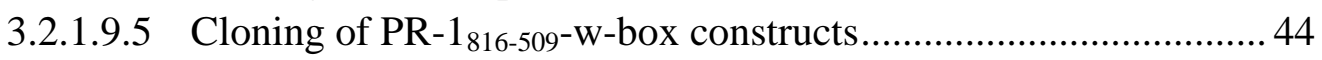

3.2.1.9.6 Klenow fragment-mediated filling of 5 end overhangs ................ 44

3.2.1.9.7 T4 polymerase-mediated filling of 5`end overhangs ................... 45

3.2.1.9.8 Radioactive labeling of EMSA fragments ................................... 45

3.2.1.9.9 Electrophoretic mobility shift assays (EMSAs) ......................... 45

3.2.1.10 Gene transfer to E. coli .................................................................. 46

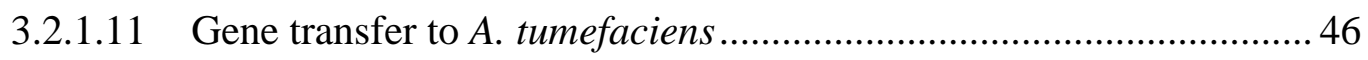

3.2.1.12 Agrobacterium- mediated gene transfer to A. thaliana ..................... 47

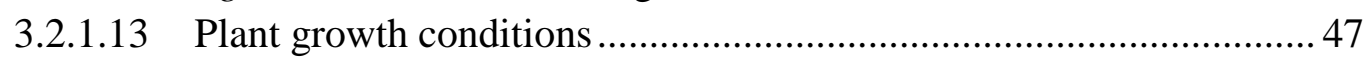

3.2.1.13.1 Growth of transgenic reporter gene lines ................................ 47

3.2.1.13.2 Plant growth conditions for transient expression studies ............ 47

3.2.1.13.3 Plant growth conditions for EMSA studies.............................. 47

3.2.1.14 RNA extraction........................................................................... 48

3.2.1.15 Quantitative Realtime RT-PCR (qRT-PCR) ................................... 48

3.2.1.16 Preparation of protein extracts for LUC assays ................................ 49

3.2.1.17 Preparation of protein extracts for MUG assays ............................. 49

3.2.1.18 Determination of protein concentrations ........................................ 49

3.2.1.19 Measurement of RLU using the FLUOstar® Ultima platereader ..... 49

3.2.1.20 Measurement of relative GUS activities.......................................... 50

3.2.1.21 Transient expression analysis by protoplast transformation ..............50

3.2.1.22 Buffers used for protoplast transformations .................................. 50

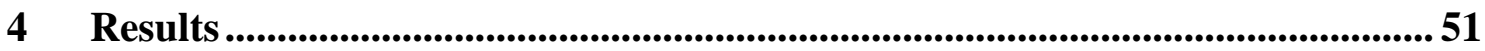

4.1 The $P R-1$ promoter contains at least two different integration sites for SNI1 -

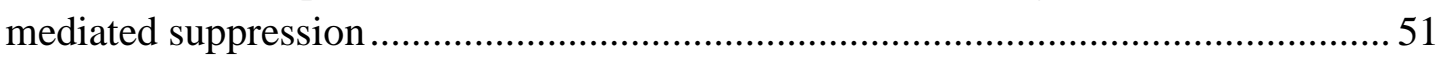

4.2 W-boxes downstream of the $L S$-region contribute to $P R-1$ induction and

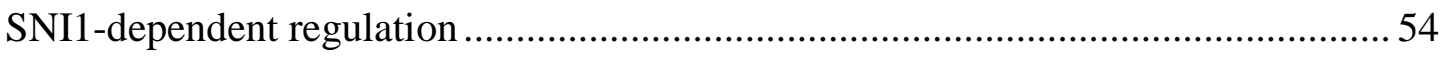

4.3 The as-1-like element is dispensable for INA induced activation of $P R-1 \ldots 55$

4.4 Alteration of the distance between the palindromic repeats of the as-1-like element interferes with INA induced expression 
4.5 The CaMV 35S as-1 element confers deregulated high expression in the $P R-1$ promoter context

4.6 The sequence between the TGA binding sites of the as- 1 element is critical for the increased constitutive expression observed in the as-1 reporter plants 65

4.7 The first base within the $a s-1$ linker region determines the overexpression capacity of the as-1 promoter construct.

4.8 Substitution of the as- 1 element within the $P R-1$ promoter changes the influence of subgroup II TGA factors on expression

4.9 In vitro binding studies reveal a similar recruitment of TGA factors to the $35 \mathrm{~S}$

as-1- and as-1-like element

4.10 LS10 contributes to $P R-1$ induction in wt and snil-1....

4.11 The cis-elements LS4 and LS10 contribute to the overall overexpression competence of the substituted $35 S$ as- 1 element

5 Discussion

5.1 A W-box cluster enhances $P R-1$ expression and integrates signals of SNI1repressed activators

5.2 The $P R-1$ promoter is regulated by an as- 1 -dependent- and $a s-1$-independent induction pathway

5.3 The as-1-independent pathway requires NPR1

5.4 SNI1 might be the repressor acting at $L S 5$ to repress the $a s-1$-independent pathway

5.5 LS4 is a positive element in the absence, and a negative element in the presence of $L S 5$

5.6 The LS7 element is essential for $P R-1$ induction in snil-1

5.7 $L S 7$ and $L S 10$ are potentially both involved in TGA factor recruitment and

NPR1 mediated activation

5.8 PR-1 regulation in snil-1 and snil nprl mutant background

5.9 An activator is recruited to the $35 S$ as -1 element in the $P R-1$ promoter context 90

5.10 In the absence of subclass II TGA factors a negative regulator is recruited to the $35 S$ as- 1 element

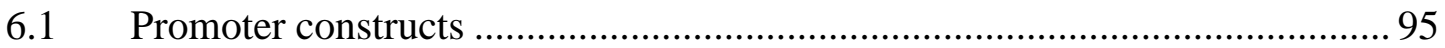

6.2 Binary vectors used for transgenic- and transient reporter gene studies 96 


\begin{tabular}{|c|c|}
\hline Contents & IV \\
\hline 6.3 & Overview of relative promoter activities ................... \\
\hline 6.3 .1 & $1 \quad L S$-constructs (pBGWL7) ......................... \\
\hline 6.3 .2 & 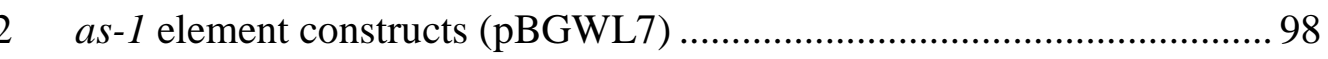 \\
\hline 6.3 .3 & 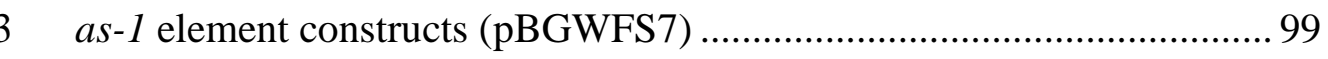 \\
\hline 6.3 .4 & 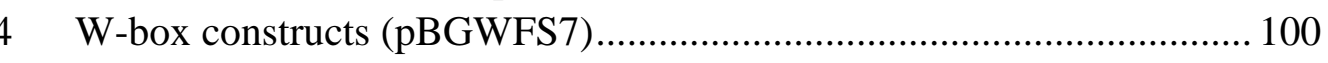 \\
\hline 6.3 .5 & 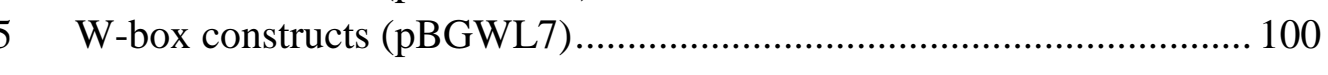 \\
\hline 6.4 & Overview of the LS-construct promoter activities in wt and snil-1........... 101 \\
\hline $\begin{array}{ll}6.5 \\
\text { inducin }\end{array}$ & $\begin{array}{l}\text { Expression of WRKY46, WRKY53 and WRKY70 in wt and snil-1 under } \\
\text { ag- and non-inducing conditions }\end{array}$ \\
\hline
\end{tabular}

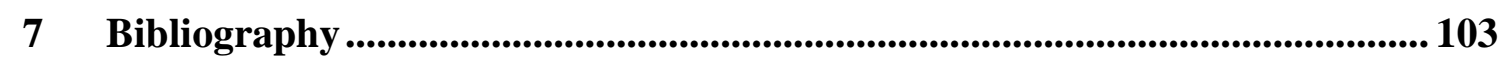

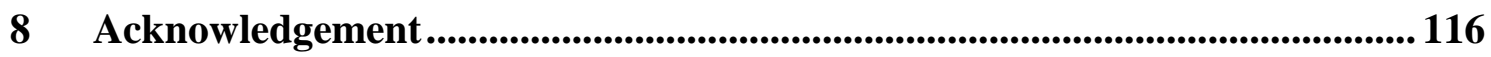

9 Curriculum Vitae ............................................................................................... 117 


\section{Abbreviations}

2,4-D

aa

A

A

ABA

amp

APS

as-1

AT

A. thaliana

A. tumefaciens

BLAST

bp

BSA

bZIP

C

CaMV

CAT

cDNA

ChIP

C-terminal

Da

ddNTPs

DMSO

DNA

DNase

dNTP 2,4-dichlorophenoxyacetic acid

amino acids

Ampere

adenosine

abscisic acid

ampicilin

ammoniumpersulfate

activating sequence 1

marks a protein from Arabidopsis thaliana

Arabidopsis thaliana

Agrobacterium tumefaciens

basic local alignment search tool

base pairs

bovine serum albumine

basic leucine zipper

cytosine

cauliflower mosaic virus

catalase

copy DNA

chromatin immunoprecipitation

carboxy-terminal

Dalton

didesoxy nucleotides

dimethylsulfoxide

desoxyribonucleic acid

desoxyribonuclease

desoxyribonucleotides 


\begin{tabular}{|c|c|}
\hline DTT & dithiotreitol \\
\hline $\mathrm{EB}$ & elusion buffer \\
\hline E. coli & Escherichia coli \\
\hline EDTA & ethylenediaminetetraacetic acid \\
\hline EMSA & Electrophoretic mobility shift assay \\
\hline ET & ethylene \\
\hline et al. & et alii (and others) \\
\hline $\mathrm{EtOH}$ & ethanol \\
\hline $\mathrm{F}$ & Farad \\
\hline$g$ & gravitation \\
\hline$g$ & gram \\
\hline $\mathrm{G}$ & guanine \\
\hline GA & gibberellic acid \\
\hline$\beta-\mathrm{Gal}$ & $\beta$-galactosidase \\
\hline GFP & green fluorescence protein \\
\hline GST (U) & glutathione-S-transferase ( $\tau$-class) \\
\hline GUS & glucuronidase \\
\hline $\mathrm{h}$ & hours \\
\hline HIS & marker gene for histidine prototrophy \\
\hline $\mathrm{H}_{2} \mathrm{O}_{2}$ & hydrogen peroxide \\
\hline hpi & hours past infection \\
\hline HR & hypersensitive response \\
\hline HSP & herring sperm DNA \\
\hline IAA & auxin \\
\hline INA & isonicotinic acid \\
\hline JA & jasmonic acid \\
\hline $\mathrm{k}$ & kilo $\left(10^{3}\right)$ \\
\hline kan & Kanamycine \\
\hline $\mathrm{L}$ & litre \\
\hline
\end{tabular}




\begin{tabular}{|c|c|}
\hline $\operatorname{lac} Z$ & gene coding for $\beta$-galactosidase \\
\hline LB & left border \\
\hline LUC & Luciferase \\
\hline$\mu$ & $\operatorname{micro}\left(10^{-6}\right)$ \\
\hline $\mathrm{m}$ & mili $\left(10^{-3}\right)$ \\
\hline M & molarity $[\mathrm{mol} / \mathrm{L}]$ \\
\hline Me-SA & methyl salicylate \\
\hline $\min$ & minutes \\
\hline mRNA & messenger RNA \\
\hline MS & Murahige and Skoog \\
\hline $\mathrm{n}$ & nano $\left(10^{-9}\right)$ \\
\hline NPR1 & NON-EXPRESSOR of $P R$-GENES 1 \\
\hline$\Omega$ & Ohm \\
\hline OD & optical density \\
\hline OE-PCR & overlap extension polymerase chain reaction \\
\hline $\mathrm{o} / \mathrm{n}$ & over night \\
\hline $\mathrm{p}$ & $\operatorname{pico}\left(10^{-12}\right)$ \\
\hline PAA & polyacrylamide \\
\hline PAGE & polyacrylamide gelelectrophoresis \\
\hline PCR & polymerase chain reaction \\
\hline PCD & programmed cell death \\
\hline PDA & potato dextrose agar \\
\hline PDB & potato dextrose broth \\
\hline $\mathrm{pH}$ & negative $\log _{10}$ of proton concentration \\
\hline PMSF & phenyl-methyl-sulfonyl-fluoride \\
\hline$P R$ & Pathogenesis related \\
\hline$P R-1$ & pathogenesis-related protein 1 \\
\hline RNA & ribonucleic acid \\
\hline RNase & ribonuclease \\
\hline
\end{tabular}




\begin{tabular}{|c|c|}
\hline ROS & reactive oxygen species \\
\hline $\mathrm{rpm}$ & rotations per minute \\
\hline RT & room temperature \\
\hline s & second \\
\hline SA & salicylic acid \\
\hline SAR & systemic acquired resistance \\
\hline SDS & sodium dodecylsulfate \\
\hline SNI1 & SUPPRESSOR OF NPR1, INDUCIBLE 1 \\
\hline SOD & superoxide dismutase \\
\hline spec & Spectinomycine \\
\hline $\mathrm{T}$ & thymine \\
\hline TE & tris-EDTA buffer \\
\hline TEMED & $\mathrm{N}, \mathrm{N}, \mathrm{N}^{\prime}, \mathrm{N}^{\prime}$-tetraethylenediamine \\
\hline TGA & as- 1 (TGACG motive) binding bZIP transcription factors \\
\hline TIBA & 2,3,5-triiodobenzoic acid \\
\hline Tris & tris-hydroxymethylamino methane \\
\hline $\mathrm{u}$ & unit (quantity for enzyme activity) \\
\hline $\mathrm{U}$ & uracil \\
\hline UV & ultra violet \\
\hline V & Volt \\
\hline $\mathrm{v} / \mathrm{v}$ & volume per volume \\
\hline $\mathrm{W}$ & Watt \\
\hline wt & wildtype plants \\
\hline $\mathrm{w} / \mathrm{v}$ & weight per volume \\
\hline X-Gal & 5-bromo-4-chloro-3-indolyl- $\beta$-D-galactopyranoside \\
\hline
\end{tabular}




\section{Summary}

The Arabidopsis $P R-1$ gene belongs to a subset of genes upregulated during "systemic acquired resistance" (SAR), a plant defense response against a broad spectrum of pathogens mediated by the signaling molecule salicylic acid (SA). Genetic analysis has revealed that the promoter is repressed by SNI1 and that this repression has to be overcome by the SA-sensitive positive regulator NPR1. NPR1 activates transcription after association with TGA factors which bind to the as-1-like element of the $P R-1$ promoter. This study reveals that NPR1 and SNI1 regulate the $P R-1$ promoter through the as-1-like element and through WRKY boxes. The SNI1/NPR1 antagonism at the $P R-1$ promoter is explained by the existence of two alternate activation pathways. In the wildtype promoter, activation is mediated by the TGA-NPR1 complex at the as-1-like element. The as-1-like element represses at the same time the alternate activation pathway, presumably through indirect interactions with SNI1. In the absence of the as-1 element or SNI1, the alternate pathway can be activated through WRKY transcription factors, which are expressed in an NPR1-dependent manner. NPR1 cannot activate the promoter if the distance between the two TGA binding sites of the as-1-like element is 4- instead of 9bps, but such an as-1-like element can still repress the alternate pathway. The as- 1 element of the CaMV $35 S$ promoter, which contains $4 \mathrm{bps}$ between the TGA binding sites, confers strong constitutive activation when replacing the as-1-like element of the $P R-1$ promoter, as a binding site for a strong activator is encoded in the sequence of this cis-regulatory element. Thus, the as-1 element of the CaMV $35 S$ promoter cannot mediate repression of the alternate pathway in the $P R-1$ promoter context. However, the repression capacity is re-established in the absence of the TGA subclass II members TGA2, TGA5 and TGA6. 


\section{Introduction}

\subsection{Plant immune responses}

Plant-microbe interactions display a well-studied example for co-evolutionary development, as the strong selective pressure favors constant modulations of attack- and counterattack mechanisms (Chisholm et al. 2006). The most common form of plant defense response against a wide range of microorganisms is referred to as "non-host resistance" (Lipka et al. 2008). This kind of defense mechanism protects the plant by preventing the penetration or the establishment of the pathogen in early phases of attack and thereby prevents infection. Plants encountered with non-host pathogens show no visible symptoms at all (type I non-host resistance) or a rapid induction of cell death (type II non-host resistance) (Mysore and Ryu 2004). Important mediators involved in non-host resistance against fungi belong to the PEN (PENETRATION) class of proteins, which act at the cell periphery and limit fungal growth by execution of apoplastic immune responses (Meyer et al. 2009). The induction of essential processes concerning the establishment of the non-host resistance response can be elicited by molecules presented by a variety of microbes. These molecules are therefore designated as pathogen- or microbe-associated molecular pattern (PAMPs/MAMPs) and the induced defense response is thus frequently termed PAMP-triggered immunity (PTI) (Murray et al. 2007). Typical MAMPs are the 22-amino-acid epitope of flagellin, flg22 (Felix et al. 1999), or the most abundant bacterial protein, the elongation factor Tu (EFTu) (Kunze et al. 2004). The sensing of these substances is followed by the activation of a complex defense signaling network at the local site of infection and a subsequent activation of systemic acquired resistance (SAR), which renders the plant more resistant in distal tissues (Mishina and Zeier 2007). This barrier of inducible defense contributes to "basal resistance" of the plant, since the recognized molecular charateristics are shared by large groups of microbes and are therefore relatively unspecific with regard to the attacking pathogen. However, pathogens developed mechanisms to prevail over basal resistance by blocking essential steps in the PAMP-stimulated signaling cascade via the evolution of virulence factors referred to as effector proteins (Stahl and Bishop 
2000). A more efficient and specific second layer of defense can be triggered upon recognition of these bacterial effector proteins by specialized resistance proteins $(R$ proteins) of the plant, a process designated as $R$ gene-mediated resistance or effector triggered immunity (ETI) respectively. Sensing of bacterial effector (avr) proteins can elicit a hypersensitive response (HR), a rapid and effective defense reaction against biotrophic pathogens which is initiated by accumulation of reactive oxygen species (ROS) and subsequently causes local cell death of infected tissue. The establishment of a HR goes along with the activation of SAR (Staskawicz et al. 1995; Ryals et al. 1996; Grant and Lamb 2006). The downstream events leading to resistance against attacking pathogens are dependent on the interplay between several phytohormone pathways whose positive and negative feedback regulation is critical for the efficiency of the defense response.

\subsection{Plant hormones and their role in host defense}

The communication between plant cells and tissues is mediated via phytohormone signaling in analogy to the hormone system known from animals. Important hormonal regulators in the context of defense responses are jasmonate (JA), salicylic acid (SA) and ethylene (ET) (Glazebrook 2001; Pieterse and van Loon LC 1999; Thomma et al. 2001). Resistance is achieved by processes like cell wall strengthening, lignifications, pathogenesis-related (PR)-gene expression, phytoalexin synthesis and, in case of Rgene-mediated resistance, induction of localized cell death (Jalali et al. 2006). Although the three mentioned phytohormones all contribute to plant defense, they are functioning in an antagonistic way and their synthesis is dependent on the kind of pathogen that is attacking the plant. For instance, the SA signaling pathway plays a major role in the defense against biotrophic pathogens like Pseudomonas syringae, Pernospora parasitica and different viruses, while JA/ET signaling is activated after infection with necrotrophic pathogens or by herbivorous insects (Dong 2001; Feys and Parker 2000; Kachroo et al. 2000; Kessler and Baldwin 2002; Takahashi et al. 2002; Thomma et al. 1998). Biotrophic pathogens require living host cells to establish their propagation, whereas necrotrophic attackers obtain their nutrition from dead plant tissue. 
JA is an oxygenated fatty acid-derivative (oxylipin), which is produced via oxidative metabolism of polyunsaturated fatty acids by enzymes of the octadecanoid pathway. The synthesis of this phytohormone is induced after attack of pathogens with necrotrophic lifestyles or by wounding. Infestation of plants with the necrotrophic fungus Botrytis cinerea causes the formation of necrotic lesions due to toxic enzymes and metabolites secreted by the attacker and by elicitation of an oxidative burst during penetration (van Kan 2006). The dead tissue serves the invaded microorganism as a source for nutrients. The receptor protein of the JA conjugate JA-isoleucine (JA-Ile) is

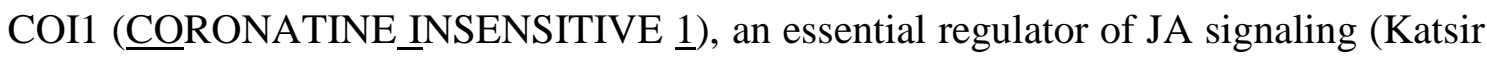
et al. 2008). coil-1 mutants exhibit increased susceptibility to necrotrophic fungi and herbivores and induction of JA-responsive marker genes like LOX and VSP or the plant defensin PDF1.2 is completely abolished (Reymond et al. 2004; Stintzi et al. 2001). The $\mathrm{COI} 1$ protein is an E3-ligase that forms the multi protein complex $\mathrm{SCF}^{\mathrm{COI} 1}$ (skip-cullin-

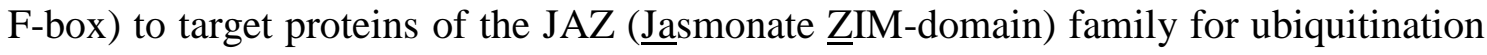
and subsequent degradation in the 26S proteasome (Chini et al. 2007; Thines et al. 2007). ET signaling also contributes to resistance against necrotrophic pathogens beside its well characterized functions in fruit ripening and senescence (Thomma et al. 1999; Yang and Hoffman 2003). Central regulators of this pathway are the ET receptors ETR1 (ENHANCED TRIPLE RESPONSE $\underline{1}$ ), the suppressor CTR1 (CONSTITUTIVE

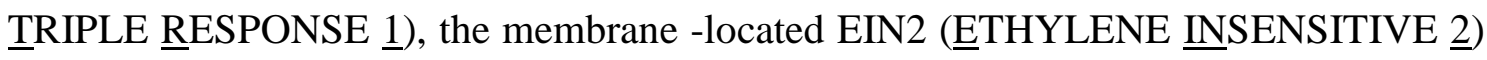
protein and the transcription factor EIN3 (ETHYLENE INSENSITIVE $\underline{3}$ ) (Chen et al. 2005; Alonso et al. 1999). CTR1 suppresses ET signaling in the absence of the hormone and is inactivated upon binding of ET to the CTR1 receptor, which subsequently leads to a MAP-kinase (mitogen-activated protein)-mediated phosphorylation cascade and a stabilization of EIN3 (Gao et al. 2003). JA- and ET signaling show a synergistic crosstalk on the expression of important defense related genes like osmotins and PDF1.2 (Thomma et al. 1998).

The SA pathway is generally activated by pathogens exhibiting a biotrophic lifestyle and is crucial for basal and $R$-gene mediated resistance (Tsuda et al. 2008). Biotrophic microorganisms receive their nutrients from living plant cells and recognition by the plant leads in case of ETI to the establishment of a hypersensitive response (HR) and systemic acquired resistance (SAR). During HR, reactive oxygen species (ROS) are 
produced following programmed cell death (PCD) and disintegration of the infested tissue (Lam et al. 2001).

Due to the different kinds of defense responses required against biotrophic, hemibiotrophic and necrotrophic pathogens as well as herbivorous insects, a tightly regulated fine tuning of the hormonal pathways is essential for the fitness of the plant. Activation of the SA signaling cascade causes a negative cross-talk on JA signaling, as revealed by a lack of PDF1.2 induction after combined exogenous application of SA and JA (Kunkel and Brooks 2002). This negative regulation depends on NPR1 (NONEXPRESSOR OF PR-GENES 1), a key regulator in SA- dependent signal transduction (Spoel et al. 2003), but the NPR1 dependency is lost when ET signaling modulates the SA/JA cross-talk (Leon-Reyes et al. 2009). A positive effect of ET on SA-dependent defense signaling and strengthened accumulation of the marker gene $P R$ 1 has been described previously (De Vos et al. 2005; Lawton et al. 1994). The biological relevance of the negative influence of SA on JA-mediated resistance was shown by experiments employing biotrophic Pseudomonas syringae and necrotrophic Alternaria brassicicola in cross-talk experiments. The results revealed that pathogens with different lifestyles force the plant to mediate tradeoffs between distinct defense signaling pathways (Spoel et al. 2007). Due to the complex interplay between hormonal signaling pathways, pathogens evolved strategies to manipulate the immune response of the plant to increase pathogenicity. Some Pseudomonas syringae strains are able to produce the phytotoxin coronatine (COR) (Bender et al. 1999), a molecule that structurally resembles JA-derivates like JA-Ile (Staswick 2008). COR induces a similar subset of genes as MeJA (Uppalapati et al. 2005) and experiments with COR-deficient $P$. syringae strains and plants impaired in the JA pathway demonstrate that $P$. syringae utilizes COR to mimick JA-signaling and to suppress SA-dependent defense (Nomura et al. 2005).

Beside SA, JA and ET, there are other hormones and genes involved in plant defense. For instance the PAD3 (PHYTOALLEXIN DEFICIENT $\underline{3}$ ) gene plays an indispensible role in camalexin biosynthesis, a phytoalexin important for resistance against necrotrophic pathogens. The camalexin production is independent of SA, JA and ET and is induced by oligogalacturonides (OG) accumulating due to cell wall degradation processes caused by the pathogen (Ferrari et al. 2007). Furthermore the phytohormone 
abscisic acid (ABA) has in impact on plant defense, partly by affecting JA biosynthesis (Adie et al. 2007), as well as Auxin (IAA), which is antagonized by SA-signaling (Wang et al. 2007).

\subsection{SA signaling and systemic acquired resistance (SAR)}

SAR initiation provokes an increased protection against a wide range of microorganisms and is associated with an activation of signal transduction pathways and the induction of $P R$-genes (Uknes et al. 1992; Van Loon 1997; Durrant and Dong 2004). The onset of SAR causes an endogenous increase in salicylic acid (SA) levels in local and systemic tissues (Malamy et al. 1990), resulting in a primed defense state of the plant (Durrant and Dong 2004). Several PR-genes exhibit chitinase or glucanase activities and their synergistic action yields a strong antipathogenic potential. However, the role and molecular function of $P R-1$ remains elusive (Van Loon 1997).

SA is a critical molecule for the establishment of SAR, proven by experiments conducted with tobacco and A. thaliana plants carrying a transgenic $N a h G$ gene. $N a h G$ encodes a bacterial hydroxylase which converts SA to catechol and thereby depletes endogenous SA levels. The $N a h G$ plants are no longer able to accumulate SA and $P R-1$ transcripts after pathogen infection, resulting in an impaired SAR (Gaffney et al. 1993). The observation that SA accumulates in phloem exudates led to the hypothesis that SA is the mobile signal produced at the site of infection and transported in systemic tissues to render the whole plant more resistant to secondary pathogen attacks (Métraux et al. 1990). Tobacco grafting experiments with $N a h G$ rootstocks and wildtypic scions revealed that SA is not important for the generation of the mobile signal, but reciprocal grafting experiment illustrated the importance of SA for perceiving the mobile signal and assuring systemic resistance (Vernooij et al. 1994). A recent study has shown that a volatile ester of SA, methyl SA (MeSA), plays a critical role as mobile SAR signal, at least in tobacco plants. MeSA is synthesized at the site of infection by SA carboxyl methyltransferase (SAMT) and cleaved in systemic tissue by SA binding protein 2 (SABP2) (Park et al. 2007). The methyl esterase activity of SABP2 is essential for the perception of the signal and initiation of SAR. 
Other studies suggest a lipid-based molecule to be the decisive mobile signal in SAR.

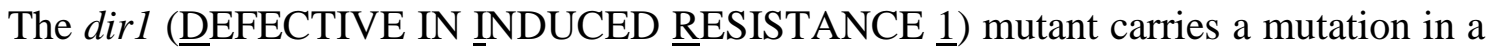
gene similar to lipid transfer proteins (LTPs) and displays normal local resistance to pathogens, while the generation of SAR and induction of $P R$-genes in systemic tissues fails (Maldonado et al. 2002).

After pathogen attack, SA is synthesized from chorismate, derived from the shikimate pathway, by isochorismate synthase (ICS1) (Wildermuth et al. 2001). The components of the shikimate pathway are transcriptionally upregulated after infection to provide sufficient amounts of chorismate for SA biosynthesis (Truman et al. 2006). The ICS1 protein shows a high chorismate binding affinity and is localized in the stroma of chloroplasts (Strawn et al. 2007). Mutation of the ICS1 protein in sid2 (ㅁA INDUCTION-DEFICIENT 2) causes a reduction of SA accumulation after infection to only $5-10 \%$ of the wildtypic level and a decrease in basal and systemic resistance (Wildermuth et al. 2001). Regulatory steps upstream of ICS1 are dependent on the attacking pathogen and the defense pathway elicited by it. In case of resistance mediated via the $R$-gene RPS4 (ㅌESISTANCE TO $\underline{P S E U D O M O N A S ~} \underline{S} Y R I N G A E \underline{4}$ ), key regulatory proteins upstream of ICS1 are the two lipase-like proteins EDS1

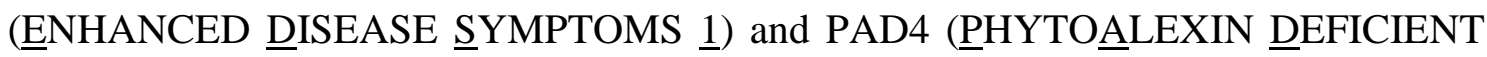
$\underline{4}$ ), which function in a positive feedback loop to increase SA biosynthesis and their own expression (Feys et al. 2001). Furthermore, the initiation of SAR and the production of SA are accompanied by the accumulation of ROS. Earlier experiments showed, that SA can directly inhibit the $\mathrm{H}_{2} \mathrm{O}_{2}$ scavanging enzymes catalase and ascorbat peroxidase (APX) (Chen et al. 1993; Durner and Klessig 1995), while later studies suggest ROS signaling upstream of SA biosynthesis (Bi et al. 1995; Neuenschwander et al. 1995). Application of high $\mathrm{H}_{2} \mathrm{O}_{2}$ concentrations to plants leads to a dose-dependent induction of SA synthesis and PR-1 expression (Leon et al. 1995). On the other hand, $\mathrm{H}_{2} \mathrm{O}_{2}$ is not detectable in systemic tissue (Ryals et al. 1995), making the attributed second messenger function of $\mathrm{H}_{2} \mathrm{O}_{2}$ questionable. Taken together, it seems that the combinatorial action of SA and ROS, produced during microburst formations, increases the defense response in systemic tissues synergistically (Du and Klessig 1997; Shirasu et al. 1997). Additionally, SA changes the intracellular redox potential, observable by alterations in absolute glutathione levels and differences in the ratio of oxidized (GSSG) 
to reduced (GSH) glutathione. In the first hours after pathogen attack or SA treatment, an initial oxidative burst occurs followed by a recovery and rebounce to a reduced environment in local as well as in systemic tissues (Mou et al. 2003). Interestingly, these changes only take place when a compatible interaction is given, illustrating the importance of the variations in redox potential for SAR (Schafer and Buettner 2001). The distinct redox states go along with the activation of different sets of target genes. During the oxidizing phase, early SA responsive genes are induced that play a role in detoxifying oxidative stress, like glutathione-S-transferases or glucosyltransferases (Blanco et al. 2009; Uquillas et al. 2004), while under later reducing conditions $P R$ gene expression takes place (Dong 2004).

SA is an electrophilic compound and high concentrations can cause detrimental effects due to xenobiotic stress. Therefore plants are able to form the bioinactive SA conjugate SA 2-o- $\beta-D-g l u c o s i d e ~(S A G)$, which can be stored in the vacuole and serves as a hydrolysable source for SA. The enzyme responsible for this conversion is the UDPglucosyltransferase (UGT) (Dean et al. 2005). The establishment of SAR and induction of $P R$-genes can be elicited by exogenous application of SA or synthetic compounds like 2,6-dichloroisonicotinic acid (INA) and benzo(1,2,3)-thiadiazole-7-carbothioic acid S-methyl ester (BTH) (Durner and Klessig 1995; Friedrich et al. 1996). The advantage of these SA-analogs is their less toxic effect on the plant. 


\subsection{NPR1-, WRKY- and SNI1 dependent SA signaling}

Mutant screens designed to identify central regulators involved in SAR downstream of SA revealed that the ankyrin repeat protein NONEXPRESSOR OF PR-GENES 1 (NPR1) is essential for SAR-mediated plant defense and $P R$-gene expression (Cao et al. 1994; Delaney et al. 1995; Glazebrook et al. 1996). NPR1 contains a BTB/POZ (Broadcomplex, Tramtrack, brick-a-brac/Poxvirus, Zinkfinger) protein-protein interaction domain, an ankyrin repeat domain, a putative nuclear localization signal and phosphorylation sites (Cao et al. 1997; Ryals et al. 1997), but a DNA binding domain is missing. Signaling by NPR1 is controlled via SA-stimulated modification of the cellular redox status, leading to a reduction of oligomeric- to monomeric NPR1. Oligomeric NPR1 is sequestered in the cytosol, whereas the monomeric NPR1 is shuttled to the nucleus (Kinkema et al., 2000; Mou et al., 2003). When the protein enters the nucleus, it is able to interact with members of the TGA basic leucine zipper (bZIP) family of transcription factors and increases their DNA binding affinity to induce target gene expression (Johnson et al. 2003). A recent study has illustrated that S-nitrosylation and thioredoxins play crucial roles in the regulation of conformational changes affecting the nuclear import and export of the NPR1 protein (Tada et al., 2008).

Oppositional observations have been made by ChIP (chromatin immunoprecipitation) experiments using a NPR1 antibody to detect protein recruitment to the $P R-1$ promoter under inducing- and non- inducing conditions. The ChIP experiments revealed constitutive recruitment of NPR1 to the $P R-1$ promoter independent of the presence of subclass II TGA transcription factors (Rochon et al. 2006). Additionally, this study showed that the protein possesses co-activator capacities due to TGA2-conditioned enhanceosome formation.

A genomic approach utilizing plants expressing an NPR1-GR (glucocorticoid receptor) fusion protein was performed to identify important regulatory components involved in direct NPR1-dependent signaling (Wang et al. 2006). The transgenic plants were treated simultaneously with cycloheximide (CHX) to suppress protein translation and dexamethasone to shuttle the fusion protein to the nucleus. Genes activated by NPR1GR were direct targets of NPR1 as protein biosynthesis and subsequent activation of secondary NPR1 dependent genes was blocked by CHX. The results obtained by 
microarray analysis illustrate that members of the WRKY protein family are transcriptionally upregulated by NPR1and that these target genes are also implicated in SAR. The WRKY family of transcription factors consists of 74 members in Arabidopsis which all contain at least one conserved WRKYGQK amino-acid DNA binding motif within their protein sequences (Eulgem and Somssich 2007). Several WRKY proteins are induced by SA and show an impact on plant defense (Dong et al. 2003; Eulgem and Somssich 2007). The putative DNA recruitment sites for WRKY proteins are W-boxes, which consist of the core-binding motif (T)(T)TGAC(C/T) (Eulgem 2005; Rushton et al. 1995). Interestingly, the induction of NPR1 itself is dependent on WRKY transcription factors, since $n p r l-1$ plants transformed with a transgenic NPR1 construct driven by an endogenous promoter lacking certain W-boxes abolish NPR1 induction and complementation of the nprl-1 phenotype (Yu et al. 2001). These results illustrate, that WRKY proteins are important regulators for upstream- and downstream events in the NPR1-dependent signaling cascade. A transcriptional profiling study uncovered an overrepresentation of $\mathrm{W}$-boxes within the promoters of genes sharing a similar induction pattern as $P R-1$, emphasizing once more the role of WRKY transcription factors in SAR (Maleck et al. 2000).

SA-mediated initiation of defense gene expression can broadly be classified in three categories, dependent on the time-point of induction and NPR1 requirement. Some early defense genes like GST6 (GLUTATHIONE- $\underline{S}$-TRANSFERASE $\underline{6}$ ) are not oblique to NPR1 and even show an increased expression in nprl mutant plants after SA treatment (Uquillas et al. 2004). These genes are often associated with TGA factor-mediated regulation and show furthermore sensitivity to $2,4 \mathrm{D}$ or xenobiotic chemicals (Chen and Singh 1999; Fode et al. 2008). Early NPR1-dependent genes are frequently related to the protein secretory pathway and contain a conserved TL1 cis-motif within their promoters. The TL1 motif is critical for NPR1-dependent regulation, though the corresponding transcription factor is still unknown (Wang et al. 2005). In the later stages of SA signaling, NPR1-dependent $P R$-proteins are induced and released in the apoplastic space due to previous activation of the secretory pathway. These late NPR1dependent transcriptional processes rely on TGA transcription factors, at least in case of $P R-1$, a marker gene for late NPR1 dependent gene expression (Zhang et al. 2003). 
A negative regulator involved in NPR1-dependent gene regulation is SNI1 ( SUPPRESSOR OF NPR1; INDUCIBLE 1 ), a repressor discovered in a mutant screen for rescued $P R$-gene inducibility in nprl mutant background. snil-1 shows increased basal- and INA-induced $P R-1$ expression levels in comparison to wildtype plants and a restored SAR (Li et al., 1999). Interestingly, the snil-1 mutation causes a specific derepression of a subset of NPR1-dependent SA-responsive genes, illustrating a tight interconnection between these two proteins (Wang et al. 2006). Like NPR1, SNI1 does not contain a DNA-binding domain and it is speculated, that the structural similarity to armadillo repeat proteins enables SNI1 to form a scaffold to interact with other proteins to suppress gene expression. Changes in $P R$-gene transcription after INA- or pathogen stimulus are accompanied with chromatin modifications. It has been shown that SNI1 seems to play an essential role in this process by snil plants revealing higher rates of activating histone modifications under uninduced conditions (Mosher et al. 2006). A mutant screen for suppressors of snil discovered RAD51D, a factor involved in DNA recombination, to be an essential regulator of NPR1-independent $P R$-gene expression. The analysis of recombination frequencies in snil and rad51d showed that both proteins fulfill dual functions by co-regulating gene expression and homologous recombination (Durrant et al. 2007). Direct evidence for SNI1 recruitment to promoter sequences via DNA-binding proteins is missing so far.

Beside the key function in SAR, NPR1 plays an important role in another induced resistance response, termed ISR (Induced systemic resistance) (Pieterse et al. 1998). ISR is induced by non-pathogenic rhizobacteria and renders the plant more resistant to a wide range of pathogens. Suprisingly, ISR is not dependent on SA-, but on JA- and ETsignaling. 


\subsection{TGA transcription factors and their role in SAR}

Taking into account that NPR1 does not contain any known DNA interaction domains, a number of yeast two hybrid screens have been performed to identify interacting transcription factors. Several proteins found in these screens belong to the group of TGA transcription factors, a subgroup of the large family of bZIP transcription factors (Jakoby et al. 2002). The name of the family is derived from the ability to bind TGACG motifs in regulatory promoter sections (Katagiri et al. 1989). From the 10 group members in Arabidopsis, TGA2, TGA3, TGA5, TGA6 and TGA7 are able to interact with NPR1 (Després et al. 2000; Kim and Delaney 2002; Zhang et al. 2003; Zhou et al. 2000), whereas TGA1 and TGA4 only show weak interaction in yeast unless two unique cysteins are reduced (Després et al. 2003). Beside TGA transcription factors, three small proteins termed NIMIN1, NIMIN2 and NIMIN3 (NIM1-INTERACTING) were also found to interact with NPR1 (Weigel et al. 2001). Overexpression of NIMIN1 leads to an attenuated expression of $P R-1$ and a compromised SAR, while nimin1 mutants display a hyperactivation of $P R-1$ though disease resistance stays unaltered. These results suggest that NIMIN proteins act as negative modulators of NPR1dependent processes (Weigel et al. 2005).

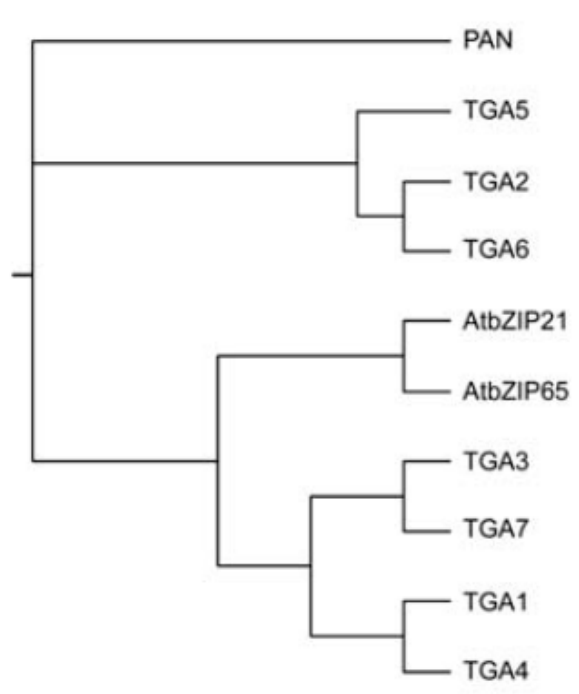

Figure2.1: Phylogenetic tree of the TGA transcription factor family in Arabidopsis thaliana (Hepworth et al. 2005).

The TGA factors 1 and 4 form the subgroup I, TGA2, 5 and 6 subgroup II, while TGA3 and TGA7 are designated as subgroup III. 
TGA factors fulfill divergent functions in the plants, as some are involved in flower development (Chuang et al. 1999) while others play a role in defense gene expression and SAR (Kesarwani et al. 2007), crosstalk (Ndamukong et al. 2007) or detoxification issues (Fode et al. 2008). PAN (PERIANTHIA) for instance is important for a proper arrangement of first whorle floral organs. Interestingly, the protein interacts with two NPR1 homologs called BOP1 and BOP2 (ㅁADE- $\underline{\text { NN}}-\underline{P E T I O L E) ~ a n d ~ p l a n t s ~ c a r r y i n g ~}$ mutations in both BOP genes show the same pentamerous flower phenotyp like pan (Hepworth et al. 2005). Another study provided evidence, that TGA4 influences flowering via interaction with CO (CONSTANS), a keyregulator of floral transition (Song et al. 2008). A further feature of TGA4 and the second subgroup I member TGA1 is the sensitivity to changes in the cellular redox state. Although both proteins show no interaction with NPR1 in yeast, the interaction takes place in planta following SA induction, linking the two TGA factor to defense gene expression and resistance. A unique trait of subgroup I members are two conserved cysteine residues (Cys260 and Cys266) which form a disulfide bound under uninduced conditions. When the redox potential changes to a more reducing environment, the disulfide bound is set off and the TGA factors are able to commit interaction with NPR1 (Després et al. 2003). Analysis of the tgal and tga4 single- and double mutants revealed an impact on basal resistance, with TGA1 playing a more dominant role in this issue (Kesarwani et al. 2007). The influence of TGA1 and TGA4 on SAR has not been subject of investigation yet.

The subclass II TGA transcription factors are redundant regulators of $P R$-gene expression and SAR. tga2 tga5 tga6 triple mutants display a similar phenotype like nprl-1 concerning a compromised SAR and an increased sensitivity to higher doses of SA (Zhang et al. 2003). Furthermore, the expression of $P R$-genes is increased under non-inducing conditions, enlightening a dual function for TGA factors as transcriptional activators and repressors (Kesarwani et al. 2007). The subclass II is also involved in NPR1-independent detoxification processes induced by electrophilic phytoprostanes, formed due to accumulation of lipophilic xenobiotic compounds or ROS (Fode et al. 2008; Mueller et al. 2008). Another regulator implicated in the NPR1-independent induction of detoxification related stress responses is the GRAS protein SCL14 (SCARECROW-LIKE 14), which interacts with subclass II TGA factors (Fode et al. 2008). ChIP experiments performed in this study revealed, that the SCL14 protein is 
recruited to target gene promoters via subclass II TGA factors and the scll4 and tga2 tga5 tga6 triple mutants show a similar negative impact on SCL14 target gene expression. Furthermore the two mutants suffer more than wildtype control plants when grown on plates supplemented with toxic INA or TIBA (2,4,6-triiodobenzoic acid) concentrations, while a SCL14 overexpressor shows enhanced resistance to the treatment. Further proteins known to interact with TGA subclass II members belong to the glutaredoxin (GRX) family. GRXs catalyze thiol disulfide reductions and are therefore implicated in regulatory processes regarding the redox state of the cell (Lemaire 2004). GRX480 is a SA-inducible glutaredoxin which antagonizes JAdependent upregulation of the defensin gene PDF1.2 in a TGA-dependent manner, linking TGA factor and GRX function to SA/JA crosstalk (Ndamukong et al. 2007).

The subclass III TGA factor TGA3 is positive regulators of $P R$-gene expression and basal resistance, shown by experiments conducted with the tga3-1 single mutant. Additionally, the increased $P R-1$ background expression observed in the tga 2 tga 5 tga6 triple mutant is vanished in the tga2 tga3 tga5 tga6 quadruple mutant, once more displaying the complex interconnection of the TGA family members (Kesarwani et al. 2007). The function of the final two TGA factors AtbZIP21 and AtbZIP65 still remains elusive, mutant analyses with regard to plant defense have not been performed yet. Due to the redundancy of different subgroup members and even different subgroups, an accurate analysis of additional multiple knock out mutants is needed to further decipher the elaborate network of TGA regulation.

\subsection{The regulatory element activating sequence 1 (as-1)}

TGA factors specifically bind to as-1-like sequences located in various defense gene promoters and activate transcription after perception of certain signals like SA and/or auxin (Liu and Lam 1994; Qin et al. 1994). bZIP proteins like TGA factors bind to DNA as hetero- or homodimers, formed by hydrophobic interactions of their leucine zipper domain (Landschulz et al. 1988). The $a s-1$ element was originally described in the Cauliflower Mosaic Virus (CaMV) 35S promoter as being a cis-regulatory element relevant for promoter activity (Benfey and Chua 1990). as-1-like elements can differ in 
the composition of their two palindromic TGAC/GTCA core-binding motifs and the distance between the two palindromic centers. SA- and auxin-responsive elements feature a $12 \mathrm{bp}$ spacing between the TGA binding motifs, whereas late SA-responsive genes like $P R-1$ show alterations in spacer length of the as-1-like element (Krawczyk et al. 2002; Strompen et al. 1998). Such differing linker regions are often correlated with NPR1-dependency of the genes, though functional data concerning this issue are missing to date (Blanco et al. 2005). Modifications in spacer length lead to changes in TGA binding affinities and thus reduce the recruitment of these factors to the corresponding cis-element (Krawczyk et al. 2002). The function of the TGA factor binding motifs of the as-1-like element of the $P R-1$ promoter differ, as revealed by analysis of promoter derivatives with substitutions in each of the two TGACG motifs. Substitution of the upper motif $L S 5$ leads to slightly enhanced basal and induced expression levels, substitution of the more downstream located LS7 leads to loss of SA sensitivity (Lebel et al. 1998). Further differences between promoters containing as-1like elements are the timing of induction after SA treatment and the need of protein biosynthesis. In contrast to $P R-1$, NPR1-independent "immediately early"-genes like GST6 or early NPR1-dependent genes like WRKY transcription factors do not need de novo protein synthesis for induction (Chen and Singh 1999; Wang et al. 2006). Another interesting aspect of TGA factor dependency with regard to the structure of the as-1 element can be observed in the tga 2 tga 5 tga6 triple mutant. While mutation of the three subgroup II members leads to an increased background expression of uninduced $P R-1$, transcription of genes comprising conserved as-1 elements in their promoters is abolished (Fode et al. 2008; Zhang et al. 2003). This result demonstrates that the structure of the TGA-binding cis-element is critical for the activating or repressing properties of the recruited TGA transcription factors. 


\begin{tabular}{|c|c|c|}
\hline Gene & AGI code & $\begin{array}{c}\text { as-1-like element } \\
\text { (-1000 bp promoter region) }\end{array}$ \\
\hline $\begin{array}{l}\text { Cytochrome P450 family protein } \\
\text { (CYP81D11) }\end{array}$ & At3g28740 & $-243 \overrightarrow{\text { TGACATATgcaaTGACGACA }}-224$ \\
\hline $\begin{array}{l}\text { SCARECROW-like transcription } \\
\text { factor } 14 \text { (SCL14) }\end{array}$ & At1g07530 & \\
\hline Oxidoreductase & At2g37770 & \\
\hline $\begin{array}{l}\text { Short-chain dehydrogenase/ } \\
\text { reductase (SDR) family protein }\end{array}$ & At4g13180 & -134 ACACGTCACtgCTTACGAAA -115 \\
\hline $\begin{array}{l}\text { 4-Methyl-5(B-hydroxyethyl)-thiazole } \\
\text { monophosphate biosynthesis protein }\end{array}$ & At3g14990 & $-71 \overrightarrow{\text { TTACGTCAtCgCACACGTCT }} \stackrel{-52}{\longleftarrow}$ \\
\hline $\begin{array}{l}\text { Glutathione S-transferase } 25 \\
\text { (GSTU7) }\end{array}$ & At2g29420 & -64 GTTCGTCACtggTGACGTCA -45 \\
\hline MtN19-like protein & At5g61820 & GAGAgcggTGACGTCA -102 \\
\hline Monooxygenase (MO1) & At4g15760 & $-107 \overrightarrow{\text { TGGCGTCAtaggCTTGCGTCA }} \quad-87$ \\
\hline $\begin{array}{l}\text { Arabidopsis NAC domain containing } \\
\text { protein } 32 \text { (ANACO32) }\end{array}$ & At1g77450 & $-206 \overrightarrow{\text { TGACGTAAg caaTGACAAAA }}-187$ \\
\hline $\begin{array}{l}\text { UDP-glucoronosyl/UDP-glucosyl } \\
\text { transferase family protein }\end{array}$ & At1g05680 & \\
\hline $\begin{array}{l}\text { Arabidopsis NAC domain containing } \\
\text { protein } 2 \text { (ANACO02, ATAF1) }\end{array}$ & At1g01720 & $-179 \overrightarrow{\text { AGACGTAAgcaaTGACAACA }}-160$ \\
\hline NADP-dependent oxidoreductase & At5g16980 & 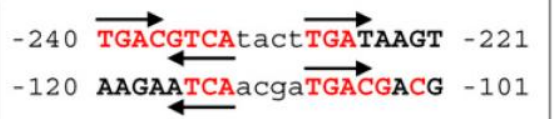 \\
\hline Nitrilase 4 (NIT4) & At5g22300 & \\
\hline $\begin{array}{l}\text { Ubiquitin carboxyl-terminal } \\
\text { hydrolase-related }\end{array}$ & At5g61950 & \\
\hline
\end{tabular}

\begin{tabular}{|l|c|}
\hline as-1:GUS & $-83 \overrightarrow{\text { TGACGTAAgggaTGACGCAC }}-64$ \\
\hline consensus & $\overrightarrow{\text { TGACGTCAnnnnTGACGTCA }}$ \\
\hline
\end{tabular}

$P R-1$ as-1-like element

\begin{tabular}{|l|l|} 
At2g14610 & $-665 \overrightarrow{\text { CTACGTCActattttacTTACGTCA }}-641$ \\
\hline
\end{tabular}

Figure 2.2: as-1 element sequences of SCL14 target genes and the as-1-like element sequence of the PR-1 promoter (Fode et al. 2008; modified).

The NPR1-independent target genes of SCL14 contain $a s-1$ elements with a conserved spacer length of $12 \mathrm{bp}$, similar to the CaMV $35 S$ as- 1 element (as-1:GUS), while the NPR1-dependent $P R-1$ gene features an $a s$-1-like element with increased distances between the palindromic repeats. 


\subsection{Aim of the study}

The signal transduction mechanism leading to the expression of $P R-1$ is of major interest for the understanding of NPR1-mediated regulation and has been subject of several studies in the last couple of years (Després et al. 2000; Kesarwani et al. 2007; Johnson et al. 2003; Rochon et al. 2006). Although much progress concerning NPR1, TGA and SNI1 biochemistry has been made, the function of cis-regulatory sequences in NPR1-dependent- and independent regulation has still to be elucidated in more detail. It has been published previously, that distinct cis-elements within the $P R-1$ promoter are essential for $P R-1$ induction (Lebel et al., 1998). Substitution of a palindromic TGA factor binding motif (LS7) by a linker sequence causes a complete loss of INAsensitivity, while other cis-element substitutions increase reporter gene expression after induction. Based on these observations, several models have been proposed which describe the regulation of $P R-1$ via recruitment of NPR1, SNI1 and TGA factors to distinct cis-elements located in the $P R-1$ promoter (Durrant and Dong 2004; Eulgem 2005; Johnson et al. 2003; Kesarwani et al. 2007). However, a detailed analysis of cisregulatory elements within the $P R-1$ promoter considering different mutant backgrounds for a more accurate functional characterization of these sequences is missing so far. Therefore, different $P R-1$ promoter:reporter constructs carrying discrete mutations, deletions or substitutions in regulatory sequences were generated, and transformed in Col-0, nprl-1, snil-1 and snil nprl to obtain stable transgenic lines. Additionally, electrophoretic mobility shift assays (EMSAs) were performed to analyze protein recruitment to these critical promoter sections. The resulting data enhance the knowledge of $P R$-gene regulation mediated via a concerted regulatory network of NPR1, SNI1, TGA factors and additional NPR1-independent proteins. 


\section{$3 \quad$ Materials and methods}

\subsection{Materials}

\subsubsection{Devices}

\begin{tabular}{|c|c|c|}
\hline Device & Model & Source \\
\hline Autoclave & $3870 \mathrm{ELV}$ & Tuttnauer \\
\hline Automatic pipettes & & Gilson \\
\hline Blotting device & & University of Göttingen \\
\hline $\begin{array}{l}\text { Chambers for gel } \\
\text { electrophoreses }\end{array}$ & & University of Göttingen \\
\hline Cooling centrifuge & Sorvall RC 5B Plus & DuPont \\
\hline $\begin{array}{l}\text { Cooling micro centrifuge } \\
\text { with overhung rotor }\end{array}$ & & Hettich \\
\hline Counting chamber & Fuchs-Rosenthal & Brand \\
\hline Electroporator & Gene Pulser ${ }^{\circledR}$ II & BioRad \\
\hline Fluorometer & CytoFluorII Plate Reader & PerSeptive \\
\hline Gel documentation device & & MWG Biotech \\
\hline Heat block & & Boekel Scientific \\
\hline Heated stirrer & RCT basic & IKA Labortechnik \\
\hline Heated shaker & Thermomixer 5436 & Eppendorf \\
\hline Locker for incubations & & WTC binder; Memmert \\
\hline Luminometer & FLUOstar Optima & BMG Labtech \\
\hline Micro centrifuge & Biofuge pico & Heraeus Christ \\
\hline Micro centrifuge, cooled & 5403 & Eppendorf \\
\hline PCR cycler & MiniCycler $^{\mathrm{TM}}$ PTC-150 & MJ Research \\
\hline pH-Meter & HI 9321 & Hanna Instruments \\
\hline Photometer & Unikon 720 LC & Kontron \\
\hline Photometer for microtiter & MRX Dynex Plate Reader & Dynex \\
\hline
\end{tabular}




\begin{tabular}{|l|l|l|}
\hline Device & & \\
\hline plates & iCycler & BioRad \\
\hline Realtime PCR cycler & GeneQuant II & Pharmacia \\
\hline RNA-/DNA-Calculator & IntelliMixer & \\
\hline Rotation platform & G2505B & Agilent Technologies \\
\hline Scanner for array slides & ABI PRISM 3100 & Perkin-Elmer \\
\hline Sequencer & Microflow Laminar & Nunc \\
\hline Sterile bench & Microflow Biohazard & Nunc \\
\hline Sterile bench & FLX-20 M & Vilber Lourmat \\
\hline UV transilluminator & Option 4, Maxima & ELGA \\
\hline Water deionization device & Cyclo 1 & Roth \\
\hline Vacuum pump & L46 & Labinco BV, Niederlande \\
\hline Vortex & & \\
\hline
\end{tabular}

\subsubsection{Materials}

\begin{tabular}{|l|l|}
\hline Product & Source \\
\hline Electroporation cuvettes & BioRad \\
\hline Filter paper Miracloth & Calbiochem \\
\hline Flow paper 3MM & Whatman \\
\hline LIA- plates, white, 96 well flat- bott & Greiner bio-one \\
\hline Microtiter plates & Roth \\
\hline Para-film M & American National Can ${ }^{\text {TM }}$ \\
\hline Plastics one-way material & $\begin{array}{l}\text { Biozym; Eppendorf; Greiner; Roth; } \\
\text { Sarstedt }\end{array}$ \\
\hline PVDF membrane Immobilon ${ }^{\mathrm{TM}}$-P & Millipore \\
\hline X-ray film Cronex 5 & Agfa, Belgium \\
\hline
\end{tabular}

\subsubsection{Chemicals}

\begin{tabular}{|c|c|}
\hline Chemical & Source \\
\hline $\begin{array}{l}30 \% \quad(w / v) \quad \text { Acrylamide: } \quad N^{\prime}, N^{\prime}- \\
\text { Methylenebisacrylamide }(37,5: 1)\end{array}$ & Roth \\
\hline
\end{tabular}




\begin{tabular}{|c|c|}
\hline Chemical & Source \\
\hline Agarose SeaKem LE & Biozym \\
\hline Ampicillin & AGS \\
\hline APS (Ammonium persulfate) & Biometra \\
\hline Bradford-Reagent & Roth \\
\hline Bromophenol blue & Roth \\
\hline BSA & Serva \\
\hline Diethylpyrocarbonat (DEPC) & Roth \\
\hline Dimethyl sulfoxide (DMSO) & Sigma \\
\hline Dithiothreitol (DTT) & Sigma \\
\hline dNTPs & MBI; Roth \\
\hline Ethylene diaminetetraacetate (EDTA) & AppliChem \\
\hline Ethidiumbromide & Roth \\
\hline Gentamycine & Duchefa \\
\hline PIPES & Roth \\
\hline Hydrogen peroxide & Roth \\
\hline Herring sperm DNA (HSP) & Sigma \\
\hline Isonicotinic acid (INA) & Sigma \\
\hline Isopropylthiogalactosid (IPTG) & Bio Tech Trade \\
\hline Kanamycine & Sigma \\
\hline$\beta$-Mercaptoethanol & Roth \\
\hline MES & Roth \\
\hline Murashige and Skoog medium & Duchefa \\
\hline Orange $\mathrm{G}$ & Sigma \\
\hline Phenol & Sigma \\
\hline Phenylmethane sulfonylchloride (PMSF) & Fluka \\
\hline Ponceau S & Sigma \\
\hline Rifampicine & Duchefa \\
\hline X-ray developer LX24 & Kodak \\
\hline X-ray fixer AL4 & Kodak \\
\hline Salicylic acid (SA) & Merck \\
\hline Select Agar & Life Technologies \\
\hline
\end{tabular}




\begin{tabular}{|l|l|}
\hline Chemical & Source \\
\hline Select Yeast Extract & GIBCO BRL \\
\hline TEMED & Roth \\
\hline Triton X-100 & Roth \\
\hline Trypton & Oxoid \\
\hline Tween20 & Roth \\
\hline X-Gal & Bio Tech Trade \\
\hline X-Gluc & Roth \\
\hline
\end{tabular}

\subsubsection{Kits}

\begin{tabular}{|l|l|}
\hline Kit & Source \\
\hline BCA Protein Assay Kit & Thermo Scientific \\
\hline $\begin{array}{l}\text { BigDye }^{\mathrm{TM}} \text { Terminator Cycle Sequencing } \\
\text { Ready Reaction Kit v.3.1 and v.1.1 }\end{array}$ & Perkin-Elmer Corporation \\
\hline HiDi-Mix & ABI PRISM \\
\hline iProof ${ }^{\mathrm{TM}}$ high fidelity PCR kit & \\
\hline Nucleo Spin ${ }^{\circledR}$ Extract II & Bio-Rad \\
\hline Nucleo Spin ${ }^{\circledR}$ Plasmid & Macherey-Nagel \\
\hline $\begin{array}{l}\text { Qiagen Plasmid Preparation Kits (Midi, } \\
\text { Maxi) }\end{array}$ & Qiagen \\
\hline QIAprep Spin Miniprep Kit & Macherey-Nagel \\
\hline QIAquick Gel Extraction Kit & Qiagen \\
\hline QIAquick PCR Purification Kit & Qiagen \\
\hline Micro Spin ${ }^{\text {TM }}$ G25 & Piagen \\
\hline
\end{tabular}




\subsubsection{Enzymes}

\begin{tabular}{|l|l|}
\hline Enzyme & Source \\
\hline Biotaq DNA polymerase & Bioline \\
\hline Cellulase “Onozuka R-10" & Serva \\
\hline Immolase DNA polymerase & Bioline \\
\hline iProof high fidelity DNA polymerase & BioRad \\
\hline Klenow DNA polymerase exo- & MBI Fermentas \\
\hline Lyticase & Sigma \\
\hline Macerozyme R-10 & Serva \\
\hline Reverse transcriptase H- & MBI Fermentas \\
\hline Restriction enzymes & MBI Fermentas, New England Biolabs \\
\hline RNase A (DNase-free) & Qiagen \\
\hline RNase inhibitor & MBI Fermentas \\
\hline T4 DNA-ligase & MBI Fermentas \\
\hline T4 DNA-polymerase & MBI Fermentas \\
\hline
\end{tabular}

\subsubsection{Standards}

\begin{tabular}{|l|l|}
\hline Standard & Source \\
\hline GeneRuler DNA Ladder Mix & MBI Fermentas \\
\hline
\end{tabular}

\subsubsection{Antibodies}

\begin{tabular}{|c|c|c|c|}
\hline Antibody & Specificity & Properties & Reference \\
\hline $\begin{array}{l}\text { } 2 \text { TGA2/5 (serum) } \\
\text { (SA 4364) }\end{array}$ & $\begin{array}{l}\text { TGA2 and TGA5 } \\
\text { C-terminal region } \\
\text { including the } \\
\text { zipper domain }\end{array}$ & $\begin{array}{l}\text { polyclonal from } \\
\text { rabbit } \\
\text { bleeding) }(1: 1000)\end{array}$ & (Fode et al. 2008) \\
\hline
\end{tabular}




\subsubsection{Nucleic acids}

\subsubsection{Primers and Oligos}

\begin{tabular}{|c|c|c|}
\hline Primer & Sequence $5^{\prime} \rightarrow 3^{\prime}$ & $\mathbf{T m}^{\circ} \mathbf{C}$ \\
\hline 3`D Oligo ChIP-R + w1+w2 F & $\begin{array}{l}\text { TGAAACACTAAGAAACAAATAATTCTTGACTTTTT } \\
\text { TTCTTTTATTTGAAAATTGACTGTAGTTGCA }\end{array}$ & $82^{\circ} \mathrm{C}$ \\
\hline $\begin{array}{l}\text { 3`D Oligo ChIP-R + w1+w2 } \\
R\end{array}$ & $\begin{array}{l}\text { ACTACAGTCAATTTTCAAATAAAAGAAAAAAAGTC } \\
\text { AAGAATTATTTGTTTCTTAGTGTTTCATGCA }\end{array}$ & $82^{\circ} \mathrm{C}$ \\
\hline $\begin{array}{l}\text { 3`D Oligo ChIP-R + } \\
\text { w1 } 1+w 2+w 3 F\end{array}$ & $\begin{array}{l}\text { TGAAACACTAAGAAACAAATAATTCTTGACTTTTT } \\
\text { TTCTTTTATTTGAAAATTGACTGTAGATATAAACTT } \\
\text { TTATTTTTTCTGACTGTATTGCA }\end{array}$ & $86^{\circ} \mathrm{C}$ \\
\hline $\begin{array}{l}\text { 3`D Oligo ChIP-R + } \\
w 1+w 2+w 3 R\end{array}$ & $\begin{array}{l}\text { ATACAGTCAGAAAAAATAAAAGTTTATATCTACAG } \\
\text { TCAATTTTCAAATAAAAGAAAAAAAGTCAAGAATT } \\
\text { ATTTGTTTCTTAGTGTTTCATGCA }\end{array}$ & $86^{\circ} \mathrm{C}$ \\
\hline 3`D Oligo Chip-R + w1F & $\begin{array}{l}\text { TGAAACACTAAGAAACAAATAATTCTTGACTTTTT } \\
\text { TGCA }\end{array}$ & $72^{\circ} \mathrm{C}$ \\
\hline 3`D Oligo Chip-R + w1R & $\begin{array}{l}\text { AAAAAGTCAAGAATTATTTGTTTCTTAGTGTTTCA } \\
\text { TGCA }\end{array}$ & $72^{\circ} \mathrm{C}$ \\
\hline 3`D Oligo ChIP-R F2 & TGAAACACTAAGAAACAAATAATTTTGCA & $63^{\circ} \mathrm{C}$ \\
\hline 3`D Oligo ChIP-R R & AAATTATTTGTTTCTTAGTGTTTCATGCA & $63^{\circ} \mathrm{C}$ \\
\hline 35S-35Slinker-PR1-F & $\begin{array}{l}\text { GACTGTTTCTTGACGTAAGGGATTACGTCATAGA } \\
\text { TGTGGCGGCATATATTCTTCAGG }\end{array}$ & $86,5^{\circ} \mathrm{C}$ \\
\hline 35S-35Slinker-PR1-R & $\begin{array}{l}\text { GCCACATCTATGACGTAATCCCTTACGTCAAGAA } \\
\text { ACAGTCAATAGATCACCCATTGAG }\end{array}$ & $86,5^{\circ} \mathrm{C}$ \\
\hline 35S-AS1-AGGA-F & $\begin{array}{l}\text { GACTGTTTCTTGACGTAAAGGATGACGCACTAGA } \\
\text { TGTGGCGGCATATATTCTTCAGG }\end{array}$ & $88^{\circ} \mathrm{C}$ \\
\hline 35S-AS1-AGGA-R & $\begin{array}{l}\text { GCCACATCTAGTGCGTCATCCTTTACGTCAAGAA } \\
\text { ACAGTCAATAGATCACCCATTGAG }\end{array}$ & $88^{\circ} \mathrm{C}$ \\
\hline 35S-AS1-F & $\begin{array}{l}\text { GACTGTTTCTTGACGTAAGGGATGACGCACTAGA } \\
\text { TGTGGCGGCATATATTCTTCAGG }\end{array}$ & $89^{\circ} \mathrm{C}$ \\
\hline 35S-AS1-R & $\begin{array}{l}\text { GCCACATCTAGTGCGTCATCCCTTACGTCAAGAA } \\
\text { ACAGTCAATAGATCACCCATTGAG }\end{array}$ & $89^{\circ} \mathrm{C}$ \\
\hline 35S-AS1-GAAA-F & $\begin{array}{l}\text { GACTGTTTCTTGACGTAAGAAATGACGCACTAGA } \\
\text { TGTGGCGGCATATATTCTTCAGG }\end{array}$ & $87^{\circ} \mathrm{C}$ \\
\hline 35S-AS1-GAAA-R & $\begin{array}{l}\text { GCCACATCTAGTGCGTCATTTCTTACGTCAAGAA } \\
\text { ACAGTCAATAGATCACCCATTGAG }\end{array}$ & $87^{\circ} \mathrm{C}$ \\
\hline
\end{tabular}




\begin{tabular}{|c|c|c|}
\hline Primer & Sequence $5^{\prime} \rightarrow 3^{\prime}$ & $\operatorname{Tm}^{\circ} \mathbf{C}$ \\
\hline 35S-AS1-GAGA-F & $\begin{array}{l}\text { GACTGTTTCTTGACGTAAGAGATGACGCACTAGA } \\
\text { TGTGGCGGCATATATTCTTCAGG }\end{array}$ & $88^{\circ} \mathrm{C}$ \\
\hline 35S-AS1-GAGA-R & $\begin{array}{l}\text { GCCACATCTAGTGCGTCATCTCTTACGTCAAGAA } \\
\text { ACAGTCAATAGATCACCCATTGAG }\end{array}$ & $88^{\circ} \mathrm{C}$ \\
\hline 35S-AS1-PR1 linker-F & $\begin{array}{l}\text { GACTGTTTCTTGACGTAACTATTTTACTGACGCA } \\
\text { CTAGATGTGGCGGCATATATTCTTCAGG }\end{array}$ & $86,5^{\circ} \mathrm{C}$ \\
\hline 35S-AS1-PR1linker-R & $\begin{array}{l}\text { GCCACATCTAGTGCGTCAGTAAAATAGTTACGTC } \\
\text { AAGAAACAGTCAATAGATCACCCATTGAG }\end{array}$ & $86,5^{\circ} \mathrm{C}$ \\
\hline 35Slinker-F & $\begin{array}{l}\text { GACTGTTTCTCTACGTCAGGGATTACGTCATAGA } \\
\text { TGTGGCGGCATATATTCTTCAGG }\end{array}$ & $87^{\circ} \mathrm{C}$ \\
\hline 35Slinker-R & $\begin{array}{l}\text { GCCACATCTATGACGTAATCCCTGACGTAGAGAA } \\
\text { ACAGTCAATAGATCACCCATTGAG }\end{array}$ & $87^{\circ} \mathrm{C}$ \\
\hline 35Slinker-variationB-F & $\begin{array}{l}\text { GACTGTTTCTCTACGTCAGAAATTACGTCATAGA } \\
\text { TGTGGCGGCATATATTCTTCAGG }\end{array}$ & $85^{\circ} \mathrm{C}$ \\
\hline 35Slinker-variationB-R & $\begin{array}{l}\text { GCCACATCTATGACGTAATTTCTGACGTAGAGAA } \\
\text { ACAGTCAATAGATCACCCATTGAG }\end{array}$ & $85^{\circ} \mathrm{C}$ \\
\hline 35Slinker-variation-F & $\begin{array}{l}\text { GACTGTTTCTCTACGTCAAAAATTACGTCATAGAT } \\
\text { GTGGCGGCATATATTCTTCAGG }\end{array}$ & $85^{\circ} \mathrm{C}$ \\
\hline 35Slinker-variation- $\mathrm{R}$ & $\begin{array}{l}\text { GCCACATCTATGACGTAATTTTTGACGTAGAGAA } \\
\text { ACAGTCAATAGATCACCCATTGAG }\end{array}$ & $85^{\circ} \mathrm{C}$ \\
\hline 35S-PR1linker-PR1-F & $\begin{array}{l}\text { GACTGTTTCTTGACGTAACTATTTTACTTACGTCA } \\
\text { TAGATGTGGCGGCATATATTCTTCAGG }\end{array}$ & $84^{\circ} \mathrm{C}$ \\
\hline 35S-PR1 linker-PR1-R & $\begin{array}{l}\text { GCCACATCTATGACGTAAGTAAAATAGTTACGTC } \\
\text { AAGAAACAGTCAATAGATCACCCATTGAG }\end{array}$ & $84,5^{\circ} \mathrm{C}$ \\
\hline CAT235F-Eco91I & AATGGTCACCGTGAATACCACGACGATTTCC & $76^{\circ} \mathrm{C}$ \\
\hline CAT235R-Mph1103 & CCAATGCATATCAGCACCTTGTCGCCTTGC & $78^{\circ} \mathrm{C}$ \\
\hline CAT483F & GATAGTCGACCTCAATGTACCTATAACCAGACCG & $71,5^{\circ} \mathrm{C}$ \\
\hline CAT483R & $\begin{array}{l}\text { AATGGTGACCGCAACACGCAGACGCTACTACCT } \\
\text { TCTTGCGTATAATATTTGCCCATG }\end{array}$ & $90^{\circ} \mathrm{C}$ \\
\hline ChIP-R +w1 Competition-F & $\begin{array}{l}\text { CATGAAACACTAAGAAACAAATAATTCTTGACTTT } \\
\text { TTTTC }\end{array}$ & $70,5^{\circ} \mathrm{C}$ \\
\hline ChIP-R +w1Competition-R & $\begin{array}{l}\text { GAAAAAATTCTTGACTTTATTTGTTTCTTAGTGTTT } \\
\text { CATG }\end{array}$ & $70,5^{\circ} \mathrm{C}$ \\
\hline $\begin{array}{l}\text { ChIP-R +w1mutated } \\
\text { Competition-F }\end{array}$ & $\begin{array}{l}\text { CATGAAACACTAAGAAACAAATAAAAGACTATCTT } \\
\text { TTTTC }\end{array}$ & $69^{\circ} \mathrm{C}$ \\
\hline
\end{tabular}




\begin{tabular}{|c|c|c|}
\hline Primer & Sequence $5^{\prime} \rightarrow \mathbf{3}^{\prime}$ & $\mathbf{T m}^{\circ} \mathbf{C}$ \\
\hline $\begin{array}{l}\text { ChIP-R +w1 mutated } \\
\text { Competition- } R\end{array}$ & $\begin{array}{l}\text { GAAAAAAGATAGTCTTTTATTTGTTTCTTAGTGTT } \\
\text { TCATG }\end{array}$ & $69^{\circ} \mathrm{C}$ \\
\hline ChIP-R+wrky1+2 shift-F & $\begin{array}{l}\text { AGCTTTTGCGTCTTCAAACACTAAGAAACAAATAA } \\
\text { TTCTTGACTTTTTTTCTTTTATTTGAAAATTGACTG } \\
\text { TAGAGAAGACGCAAAAG }\end{array}$ & $86^{\circ} \mathrm{C}$ \\
\hline ChIP-R+wrky1+2 shift-R & $\begin{array}{l}\text { AATTCTTTTGCGTCTTCTCTACAGTCAATTTTCAA } \\
\text { ATAAAAGAAAAAAAGTCAAGAATTATTTGTTTCTT } \\
\text { AGTGTTTGAAGACGCAAA }\end{array}$ & $86^{\circ} \mathrm{C}$ \\
\hline DOF-F & $\begin{array}{l}\text { CTTCGTTCTAGAGGAGCCATAGGCAAGAGTGATA } \\
\text { GAGATAC }\end{array}$ & $76^{\circ} \mathrm{C}$ \\
\hline DOF-R & $\begin{array}{l}\text { САСТCTTGCCTATGGCTCСTCTAGAACGAAGAAT } \\
\text { АTATGCCGCCAC }\end{array}$ & $85^{\circ} \mathrm{C}$ \\
\hline Egfp QTect -F & ACCACTACCAGCAGAACACCC & $59^{\circ} \mathrm{C}$ \\
\hline Egfp QTect -R & GAACTCCAGCAGGACCATGTG & $60^{\circ} \mathrm{C}$ \\
\hline Egfp-R & CCTCGCCCTTGCTCACCATCC & $68,5^{\circ} \mathrm{C}$ \\
\hline GFP-R & CCGTTTACGTCGCCGTCCAGC & $69^{\circ} \mathrm{C}$ \\
\hline GUS169R & GGCGAACTGATCGTTAAAACTGC & $63^{\circ} \mathrm{C}$ \\
\hline GUS431R & GGATAGTCTGCCAGTTCAGTTCG & $61^{\circ} \mathrm{C}$ \\
\hline GUS-F & CGCTGGACTGGCATGAACTTCG & $67,5^{\circ} \mathrm{C}$ \\
\hline HA-Tag forward & ATGGCATACCCATACGACGTTCC & $65^{\circ} \mathrm{C}$ \\
\hline HA-Tag reverse & CATAGTCCGGGACGTCATAGGG & $63^{\circ} \mathrm{C}$ \\
\hline LS4-LS8 shift-F & $\begin{array}{l}\text { AGCTTTTGCGTCTTCTCTATTGACTGTTTCTCTAC } \\
\text { GTCACTATTTTACTTACGTCATAGATGTGGCGGC } \\
\text { ATGAAGACGCAAAAG }\end{array}$ & $89^{\circ} \mathrm{C}$ \\
\hline LS4-LS8 shift-R & $\begin{array}{l}\text { AATTCTTTTGCGTCTTCATGCCGCCACATCTATG } \\
\text { ACGTAAGTAAAATAGTGACGTAGAGAAACAGTCA } \\
\text { ATAGAGAAGACGCAAA }\end{array}$ & $89^{\circ} \mathrm{C}$ \\
\hline LS5-F & $\begin{array}{l}\text { AGAATAATCTAGATATATTTTACTTACGTCATAGA } \\
\text { TGTGGCGGCATATATTC }\end{array}$ & $76^{\circ} \mathrm{C}$ \\
\hline LS5-R & $\begin{array}{l}\text { CGTAAGTAAAATATATCTAGATTATTCTAGAATAT } \\
\text { AGATCACCCATTGAG }\end{array}$ & $71,5^{\circ} \mathrm{C}$ \\
\hline LS5onlyF & $\begin{array}{l}\text { GGTGATCTATTGACTGTTTCAATCTAGATATATTT } \\
\text { TACTTACGTCATAGATGTGGC }\end{array}$ & $79^{\circ} \mathrm{C}$ \\
\hline LS5onlyR & $\begin{array}{l}\text { ATATATCTAGATTGAAACAGTCAATAGATCACCCA } \\
\mathrm{T}\end{array}$ & $66,7^{\circ} \mathrm{C}$ \\
\hline
\end{tabular}




\begin{tabular}{|c|c|c|}
\hline Primer & Sequence $5^{\prime} \rightarrow 3^{\prime}$ & $\mathbf{T m}^{\circ} \mathbf{C}$ \\
\hline LS5-LS10 shift-F & $\begin{array}{l}\text { AGCTTTTGCGTCTTCTCTACGTCACTATTTTACTT } \\
\text { ACGTCATAGATGTGGCGGCATATATTCTTCAGGA } \\
\text { CTTTTCAGGAAGACGCAAAAG }\end{array}$ & $89^{\circ} \mathrm{C}$ \\
\hline LS5-LS10 shift-R & $\begin{array}{l}\text { AATTCTTTTGCGTCTTCCTGAAAAGTCCTGAAGA } \\
\text { ATATATGCCGCCACATCTATGACGTAAGTAAAAT } \\
\text { AGTGACGTAGAGAAGACGCAAA }\end{array}$ & $89^{\circ} \mathrm{C}$ \\
\hline LS5-LS7F & $\begin{array}{l}\text { GGTGATCTATTGACTGTTTCAATCTAGATATATTT } \\
\text { TACTTTTTCTAGATGGTGGC }\end{array}$ & $78,5^{\circ} \mathrm{C}$ \\
\hline LS6-9bp-F & $\begin{array}{l}\text { GACTGTTTCTCTACGTCACTCTAGATTTTACGTCA } \\
\text { TAGATGTGGCGGCATATATTCTTCAGG }\end{array}$ & $85^{\circ} \mathrm{C}$ \\
\hline LS6-9bp-R & $\begin{array}{l}\text { GCCACATCTATGACGTAAAATCTAGAGTGACGTA } \\
\text { GAGAAACAGTCAATAGATCACCCATTGAG }\end{array}$ & $86^{\circ} \mathrm{C}$ \\
\hline LS7-F & $\begin{array}{l}\text { CTTTTTCTAGATGTGTGGCGGCATATATTCTTCA } \\
\text { GGACTTTTCAGCCATAGGCAAGAG }\end{array}$ & $87^{\circ} \mathrm{C}$ \\
\hline LS7-LS5-wrky-R & $\begin{array}{l}\text { CCTGAAGAATATATGCCGCCACACATCTAGAAAA } \\
\text { AGTAAAATATATCTAGATTATTCTAG }\end{array}$ & $79^{\circ} \mathrm{C}$ \\
\hline LS7onlyR & $\begin{array}{l}\text { AATATATGCCGCCACACATCTAGAAAAAGTAAAA } \\
\text { TAGTGACGTAGAGAAACAGTCAATAG }\end{array}$ & $82^{\circ} \mathrm{C}$ \\
\hline LS7-R & $\begin{array}{l}\text { CCTGAAGAATATATGCCGCCACACATCTAGAAAA } \\
\text { AGTAAAATAGTGACGTAGAATTCTAG }\end{array}$ & $82^{\circ} \mathrm{C}$ \\
\hline LS8-F & $\begin{array}{l}\text { AGAAATCTAGAGCTATATTCTTCAGGACTTTTCAG } \\
\text { CCATAGG }\end{array}$ & $74^{\circ} \mathrm{C}$ \\
\hline LS8-R & $\begin{array}{l}\text { GTCCTGAAGAATATAGCTCTAGATTTCTATGACG } \\
\text { TAAGTAAAATAGTGACGTAGAG }\end{array}$ & $77^{\circ} \mathrm{C}$ \\
\hline PR1 ChIP-R+w1+2+3F $\Delta$ & $\begin{array}{l}\text { GAGATACTCATATGCATAAATATAATCTTAATTGC } \\
\text { CAAACTGTCCG }\end{array}$ & $76^{\circ} \mathrm{C}$ \\
\hline PR1 ChIP-R+w1+2+3R $\Delta$ & $\begin{array}{l}\text { GGCAATTAAGATTATATTTATGCATATGAGTATCT } \\
\text { CTATCACTCTTGC }\end{array}$ & $74,5^{\circ} \mathrm{C}$ \\
\hline PR1 ChIP-R-F $\Delta$ & $\begin{array}{l}\text { GAGATACTCATATGCATCTTGACTTTTTTTTCTTTT } \\
\text { ATTTGAAAATTGACTGTAG }\end{array}$ & $78^{\circ} \mathrm{C}$ \\
\hline PR1 ChIP-R-R $\Delta$ & $\begin{array}{l}\text { GAAAAAAAGTCAAGATGCATATGAGTATCTCTAT } \\
\text { CACTCTTGC }\end{array}$ & $74,5^{\circ} \mathrm{C}$ \\
\hline PR1 w1 w2R $\Delta$ & $\begin{array}{l}\text { GAAACAAATAATTATATAAACTTTTATTTTTTTCTGA } \\
\text { CTGTAAATATAATCTTAATTGCC }\end{array}$ & $74^{\circ} \mathrm{C}$ \\
\hline $\mathrm{PR} 1 \mathrm{w} 1 \mathrm{w} 2 \mathrm{R} \Delta$ & $\begin{array}{l}\text { GAAAAAATAAAAGTTTATATAATTATTTGTTTCTTA } \\
\text { GTGTTTCATGC }\end{array}$ & $71^{\circ} \mathrm{C}$ \\
\hline
\end{tabular}




\begin{tabular}{|c|c|c|}
\hline Primer & Sequence $5^{\prime} \rightarrow 3^{\prime}$ & $\operatorname{Tm}^{\circ} \mathbf{C}$ \\
\hline PR1 w1+w2+w3F $\Delta$ & $\begin{array}{l}\text { GAAACAAATAATTAAATATAATCTTAATTGCCAAA } \\
\text { CTGTCC }\end{array}$ & $70^{\circ} \mathrm{C}$ \\
\hline PR1 w1+w2+w3R $\Delta$ & $\begin{array}{l}\text { GGCAATTAAGATTATATTTAATTATTTGTTTCTTAG } \\
\text { TGTTTCATGC }\end{array}$ & $71^{\circ} \mathrm{C}$ \\
\hline PR1 w1F $\Delta$ & $\begin{array}{l}\text { GAAACAAATAATTCTTTTTATTTGAAAATTGACTGT } \\
\text { AGATATAAAC }\end{array}$ & $70^{\circ} \mathrm{C}$ \\
\hline PR1 w1R $\Delta$ & $\begin{array}{l}\text { ACAGTCAATTTTCAAATAAAAGAATTATTTGTTTC } \\
\text { TTAGTGTTTCATGC }\end{array}$ & $76^{\circ} \mathrm{C}$ \\
\hline $\mathrm{PR} 1 \mathrm{w} 2 \mathrm{~F} \Delta$ & $\begin{array}{l}\text { CTTGACTTTTATATAAACTTTTATTTTTTCTGACTG } \\
\text { TAAATATAATCTTAATTGCC }\end{array}$ & $74^{\circ} \mathrm{C}$ \\
\hline PR1 w2R $\Delta$ & $\begin{array}{l}\text { CAGTCAGAAAAAATAAAAGTTTATATAAAAGTCAA } \\
\text { GAATTATTTGTTTCTTAGTGTTTCATGC }\end{array}$ & $79^{\circ} \mathrm{C}$ \\
\hline PR1-1293F-Sall & $\begin{array}{l}\text { GATAGTCGACGTAATAATATCCTATGGTGTCATTT } \\
\text { TATAAGTTAGC }\end{array}$ & $73^{\circ} \mathrm{C}$ \\
\hline PR1-3D F & $\begin{array}{l}\text { GCAAGAGTGATAGAGATACTCATATGCATAAAAA } \\
\text { AAAAGAAAAAAATAGTTTTCAAATCTC }\end{array}$ & $79,5^{\circ} \mathrm{C}$ \\
\hline PR1-3`D R & $\begin{array}{l}\text { TTTATGCATATGAGTATCTCTATCACTCTTGCCTA } \\
\text { TGG }\end{array}$ & $71^{\circ} \mathrm{C}$ \\
\hline PR1-35Slinker variationB-F & $\begin{array}{l}\text { GACTGTTTCTCTACGTCACAAATTACGTCATAGAT } \\
\text { GTGGCGGCATATATTCTTCAGG }\end{array}$ & $85,5^{\circ} \mathrm{C}$ \\
\hline PR1-35Slinker variationB-R & $\begin{array}{l}\text { GCCACATCTATGACGTAATTTGTGACGTAGAGAA } \\
\text { ACAGTCAATAGATCACCCATTGAG }\end{array}$ & $85,5^{\circ} \mathrm{C}$ \\
\hline PR1-35Slinker variation-F & $\begin{array}{l}\text { GACTGTTTCTCTACGTCAAAAATTACGTCATAGAT } \\
\text { GTGGCGGCATATATTCTTCAGG }\end{array}$ & $85^{\circ} \mathrm{C}$ \\
\hline PR1-35Slinker variation- $R$ & $\begin{array}{l}\text { GCCACATCTATGACGTAATTTTTGACGTAGAGAA } \\
\text { ACAGTCAATAGATCACCCATTGAG }\end{array}$ & $85^{\circ} \mathrm{C}$ \\
\hline PR1-35Slinker-35S-F & $\begin{array}{l}\text { GACTGTTTCTCTACGTCAGGGATGACGCACTAGA } \\
\text { TGTGGCGGCATATATTCTTCAGG }\end{array}$ & $89^{\circ} \mathrm{C}$ \\
\hline PR1-35Slinker-35S-R & $\begin{array}{l}\text { GCCACATCTAGTGCGTCATCCCTGACGTAGAGA } \\
\text { AACAGTCAATAGATCACCCATTGAG }\end{array}$ & $89^{\circ} \mathrm{C}$ \\
\hline PR1-387F & TAAAGCCAGTGCATATCAGTAGTC & $56,5^{\circ} \mathrm{C}$ \\
\hline PR1-483R & $\begin{array}{l}\text { AATGGTGACCGCAACACGCAGACGCTACTACCT } \\
\text { TTTCAGTATACCTAATTTTGTACCG }\end{array}$ & $87^{\circ} \mathrm{C}$ \\
\hline PR1-5 UTR-pBGWFS7 & $\begin{array}{l}\text { GTAGTCTAGACATTTTTCTAAGTTGATAATGGTTA } \\
\text { TTGTTG }\end{array}$ & $69^{\circ} \mathrm{C}$ \\
\hline PR1-617F & CTTTTTGGATAAATCTCAATGGGTG & $61,5^{\circ} \mathrm{C}$ \\
\hline
\end{tabular}




\begin{tabular}{|c|c|c|}
\hline Primer & Sequence $5^{\prime} \rightarrow 3^{\prime}$ & $\operatorname{Tm}^{\circ} \mathbf{C}$ \\
\hline PR1-725F & $\begin{array}{l}\text { TATATGCATGCAGAGACCAACGAGGACTACAGG } \\
\text { TTGACTTTTTTTCTTTTATTTGA }\end{array}$ & $84^{\circ} \mathrm{C}$ \\
\hline PR1-835R & AATCGTATCGGACAGTTTGGC & $59^{\circ} \mathrm{C}$ \\
\hline PR1-PR1linker-35S-F & $\begin{array}{l}\text { GACTGTTTCTCTACGTCACTATTTTACTGACGCA } \\
\text { CTAGATGTGGCGGCATATATTCTTCAGG }\end{array}$ & $87^{\circ} \mathrm{C}$ \\
\hline PR1-PR1linker-35S-R & $\begin{array}{l}\text { GCCACATCTAGTGCGTCAGTAAAATAGTGACGTA } \\
\text { GAGAAACAGTCAATAGATCACCCATTGAG }\end{array}$ & $87^{\circ} \mathrm{C}$ \\
\hline $\begin{array}{l}w 1+w 2+w 3 / w 1+w 2+w 3 \\
\text { mutated Competition-F }\end{array}$ & $\begin{array}{l}\text { AAAGACTATCTTTTTTCTTTTAATTTGAAAAAAGACT } \\
\text { ATCGATATAAACTTTTATTTTTAAAGACTATCAA }\end{array}$ & $77,5^{\circ} \mathrm{C}$ \\
\hline $\begin{array}{l}\mathrm{w} 1+\mathrm{w} 2+\mathrm{w} 3 / \mathrm{w} 1+\mathrm{w} 2+\mathrm{w} 3 \\
\text { mutated Competition- } \mathrm{R}\end{array}$ & $\begin{array}{l}\text { TTGATAGTCTTAAAAATAAAAGTTTATATCGATAG } \\
\text { TCTTTTTTCAAATAAAAGAAAAAAGATAGTCTTT }\end{array}$ & $77,5^{\circ} \mathrm{C}$ \\
\hline $\begin{array}{l}\text { w1+w2+w3 / w1+w2mutated } \\
\text { Competition-F }\end{array}$ & $\begin{array}{l}\text { AAAGACTATCTTTTTTCTTTTTATTTGAAAAAAGACT } \\
\text { ATCGATATAAACTTTTATTTTTTCTGACTGTAA }\end{array}$ & $79^{\circ} \mathrm{C}$ \\
\hline $\begin{array}{l}\text { w1+w2+w3 / w1+w2mutated } \\
\text { Competition-R }\end{array}$ & $\begin{array}{l}\text { TTACAGTCAGAAAAAATAAAAGTTTATATCGATAG } \\
\text { TCTTTTTTCAAATAAAAGAAAAAAGATAGTCTTT }\end{array}$ & $79^{\circ} \mathrm{C}$ \\
\hline $\begin{array}{l}\text { w1+w2+w3 / w1 mutated } \\
\text { Competition-F }\end{array}$ & $\begin{array}{l}\text { AAAGACTATCTTTTTTCTTTTATTTGAAAATTGACT } \\
\text { GTAGATATAAACTTTTATTTTTTTCTGACTGTAA }\end{array}$ & $78,5^{\circ} \mathrm{C}$ \\
\hline $\begin{array}{l}\text { w1+w2+w3 /w1 mutated } \\
\text { Competition- } R\end{array}$ & $\begin{array}{l}\text { TTACAGTCAGAAAAAATAAAAGTTTATATCTACAG } \\
\text { TCAATTTTCAAATAAAAGAAAAAAGATAGTCTTT }\end{array}$ & $78,5^{\circ} \mathrm{C}$ \\
\hline wrky_shift-F & $\begin{array}{l}\text { AGCTTTTGCGTCTTCCTTGACTTTTTTTCTTTTATT } \\
\text { TGAAAATTGACTGTAGATATAAACTTTTATTTTTTC } \\
\text { TGACTGTAAAGAAGACGCAAAAG }\end{array}$ & $86^{\circ} \mathrm{C}$ \\
\hline wrky_shift-R & $\begin{array}{l}\text { AATTCTTTTGCGTCTTCTTTACAGTCAGAAAAAAT } \\
\text { AAAAGTTTATATCTACAGTCAATTTTCAAATAAAA } \\
\text { GAAAAAAAGTCAAGGAAGACGCAAA }\end{array}$ & $86^{\circ} \mathrm{C}$ \\
\hline WRKY18seq-F & CCGAAGAAGGAGGTCTCAGTTTTGG & $67^{\circ} \mathrm{C}$ \\
\hline WRKY18seq-R & CAACGCTAGTCTATGACAGCCATTAACC & $66^{\circ} \mathrm{C}$ \\
\hline WRKY46-F & $\begin{array}{l}\text { GGGGACAAGTTTGTACAAAAAAGCAGGCTCCAT } \\
\text { GATGATGGAAGAGAAACTTGTGATCAACG }\end{array}$ & $91^{\circ} \mathrm{C}$ \\
\hline WRKY46-R & $\begin{array}{l}\text { GGGGACCACTTTGTACAAGAAAGCTGGGTCCTA } \\
\text { CGACCACAACCAATCCTGTCCG }\end{array}$ & $92^{\circ} \mathrm{C}$ \\
\hline WRKY46seq-F & GAGGAAAGTATCGGAGAAGAACACAGAG & $65^{\circ} \mathrm{C}$ \\
\hline WRKY46seq-R & CGTTCTGAAAATGTTCTTCTTGTTCTCC & $65^{\circ} \mathrm{C}$ \\
\hline WRKY53-F & $\begin{array}{l}\text { GGGGACAAGTTTGTACAAAAAAGCAGGCTCCAT } \\
\text { GGAAGGAAGAGATATGTTAAGTTGGGAGC }\end{array}$ & $89,5^{\circ} \mathrm{C}$ \\
\hline
\end{tabular}




\begin{tabular}{|l|l|l|}
\hline Primer & \multicolumn{1}{|l}{ Sequence5 ${ }^{\prime} \rightarrow 3^{\prime}$} & \multicolumn{2}{c|}{ Tm $^{\circ} \mathbf{C}$} \\
\hline WRKY53-R & $\begin{array}{l}\text { GGGGACCACTTTGTACAAGAAAGCTGGGTCTTAA } \\
\text { TAATAAATCGACTCGTGTAAAAACGCG }\end{array}$ & $88^{\circ} \mathrm{C}$ \\
\hline WRKY53seq2-F & CGGCTGTTGCTGAGACTAACG & $61^{\circ} \mathrm{C}$ \\
\hline WRKY53seq2-R & ACTCGCCGTTGATAGTTCCG & $60^{\circ} \mathrm{C}$ \\
\hline WRKY53seq-F & CCTCACCGAGCGTACAACTTATTCC & $65,5^{\circ} \mathrm{C}$ \\
\hline WRKY53seq-R & CCATCATCAAGCCCATCGGTTCG & $70^{\circ} \mathrm{C}$ \\
\hline WRKY-Box 1X F & CCGGGGATCTATTGACTGTTTCG & $65^{\circ} \mathrm{C}$ \\
\hline WRKY-Box 1X R & TCGACGAAACAGTCAATAGATCC & $59^{\circ} \mathrm{C}$ \\
\hline WRKY-Box 3X F & $\begin{array}{l}\text { CCGGGGATCTATTGACTGTTTCTCTACGTCGATC } \\
\text { TATTGACTGTTTCTCTACGTCGATCTATTGACTGT } \\
\text { TTCG }\end{array}$ & $90^{\circ} \mathrm{C}$ \\
\hline WRKY-Box 3X R & $\begin{array}{l}\text { TCGACGAAACAGTCAATAGATCGACGTAGAGAAA } \\
\text { CAGTCAATAGATCGACGTAGAGAAACAGTCAATA } \\
\text { GATCC }\end{array}$ & $90^{\circ} \mathrm{C}$ \\
\hline
\end{tabular}

\subsubsection{Plasmids}

\subsection{Plasmids for promoter analysis purposes}

\begin{tabular}{|c|c|c|c|}
\hline pUC18-Entry2 & pBGWFS7 & pBGWL7 & Description \\
\hline $3 ’ D$ & Yes & Yes & $\begin{array}{l}\text { Deletion of } 502 b p \text { between }-68 b p \text { and - } \\
570 b p \text {; Insertion of a sequence derived } \\
\text { from the } C A T \text { gene between }-1294 b p \\
\text { and }-817 b p\end{array}$ \\
\hline $3 ` D+C h I P-R$ & No & Yes & $\begin{array}{l}\text { 481bp deletion between }-65 \mathrm{bp} \text { and }- \\
\text { 546bp. CAT sequence between - } \\
\text { 1294bp and }-817 \mathrm{bp}\end{array}$ \\
\hline $3 D+w 1$ & No & Yes & $\begin{array}{l}\text { The first w-box is attached to the } 3 ` D \\
+C h I P-R \text { construct }\end{array}$ \\
\hline
\end{tabular}




\begin{tabular}{|c|c|c|c|}
\hline pUC18-Entry2 & pBGWFS7 & pBGWL7 & Description \\
\hline $3 D+w 1+w 2$ & No & Yes & $\begin{array}{l}\text { The two w-boxes } w 1 \text { and } w 2 \text { are } \\
\text { attached to the } 3^{\circ} D+C h I P-R \text { construct }\end{array}$ \\
\hline $\begin{array}{l}3 D+w 1+w 2+ \\
w 3\end{array}$ & No & Yes & $\begin{array}{l}\text { All three } \mathrm{w} \text { - boxes are attached to } 3{ }^{`} D \\
+C h I P-R\end{array}$ \\
\hline 3`D as-1 linker & No & Yes & $\begin{array}{l}3 ` D M p h \text { deletion construct containing } \\
\text { a substituted as-1 linker (GGGA) from } \\
C a M V 35 S\end{array}$ \\
\hline 3`DMph & Yes & Yes & $\begin{array}{l}\text { Deletion of } 504 \mathrm{bp} \text { between }-65 \mathrm{bp} \text { and - } \\
569 \mathrm{bp} \text {; Insertion of a sequence derived } \\
\text { from the } C A T \text { gene between }-1294 \mathrm{bp} \\
\text { and }-817 \mathrm{bp} \text {; reconstituted Mph } 1103 \mathrm{I} \\
\text { restriction site }\end{array}$ \\
\hline 3`D Mph as-1 & Yes & Yes & $\begin{array}{l}3{ }^{`} D M p h \text { construct with substituted } a s- \\
1 \text { element from } C a M V 35 S\end{array}$ \\
\hline $35 S-35 S-P R-1$ & No & Yes & $\begin{array}{l}\text { The downstream TGA binding motif in } \\
\text { as- } 1 \text { is substituted against the TGA } \\
\text { binding motif from the original } P R-1 \\
\text { promoter }\end{array}$ \\
\hline$a s-1$ & Yes & Yes & $\begin{array}{l}\text { Substitution of the as-1-like element } \\
\text { against } C a M V 35 S \text { as-1 }\end{array}$ \\
\hline$a s-1-A G G A$ & No & Yes & $\begin{array}{l}\text { The sequence of the } a s-1 \text { linker region } \\
\text { is changed to AGGA }\end{array}$ \\
\hline$a s-1-G A A A$ & No & Yes & $\begin{array}{l}\text { The sequence of the } a s-1 \text { linker region } \\
\text { is changed to AGGA }\end{array}$ \\
\hline$a s-1-G A G A$ & No & Yes & $\begin{array}{l}\text { The sequence of the } a s-1 \text { linker region } \\
\text { is changed to GAGA }\end{array}$ \\
\hline as-1 linker & No & Yes & $\begin{array}{l}\text { The linker sequence between the TGA } \\
\text { binding motifs is substituted against } \\
\text { the CaMV35S linker sequence }\end{array}$ \\
\hline as-1 LS10 & No & Yes & $\begin{array}{l}\text { Mutated } L S 10 \text { element and substituted } \\
\text { as- } 1 \text { element from } C a M V 35 S\end{array}$ \\
\hline as-1-LS10 & No & Yes & $\begin{array}{l}\text { Substituted as-1 element and mutated } \\
\text { LS10 box }\end{array}$ \\
\hline CAT235 & Yes & Yes & $\begin{array}{l}235 \mathrm{bp} \text { substitution in the position - } \\
\text { 810bp to }-575 \mathrm{bp} \text {; coding sequence was } \\
\text { obtained from the chloramphenicol } \\
\text { acetyltransferase gene }(C A T)\end{array}$ \\
\hline CAT477 & No & No & $\begin{array}{l}477 \mathrm{bp} \text { substitution in the position - } \\
1294 \mathrm{bp} \text { to }-817 \mathrm{bp} \text {; the sequence was } \\
\text { obtained from the chloramphenicol } \\
\text { acetyltransferase gene }(C A T) \text {; The } \\
\text { construct was used for } 3 D \text { cloning }\end{array}$ \\
\hline$L S 10$ & Yes & Yes & $\begin{array}{l}\text { Mutation of the putative DOF } \\
\text { transcription factor binding box } L S 10\end{array}$ \\
\hline
\end{tabular}




\begin{tabular}{|c|c|c|c|}
\hline pUC18-Entry2 & pBGWFS7 & pBGWL7 & Description \\
\hline LS4 & Yes & Yes & w-box mutation $L S 4$ \\
\hline LS4-as-1 & Yes & Yes & $\begin{array}{l}\text { Mutated w-box LS4 in combination } \\
\text { with substituted } a s-1 \text { element }\end{array}$ \\
\hline LS4-as-1 & Yes & Yes & $\begin{array}{l}\text { Mutated } L S 4 \text { element and substituted } \\
a s-1 \text { element from CaMV35S }\end{array}$ \\
\hline LS4-LS5 & Yes & Yes & $\begin{array}{l}\text { w-box LS4 and TGA motif } L S 5 \\
\text { mutation }\end{array}$ \\
\hline LS4-LS5-LS7 & Yes & Yes & $\begin{array}{l}\text { w-box LS4 and both TGA binding } \\
\text { motifs of the as-1-like element are } \\
\text { substituted }\end{array}$ \\
\hline$L S 4-L S 7$ & Yes & Yes & $\begin{array}{l}\text { w-box LS4 and TGA motif } L S 7 \\
\text { mutation }\end{array}$ \\
\hline LS5 & Yes & Yes & TGA binding motif mutation $L S 5$ \\
\hline$L S 5-L S 7$ & Yes & Yes & $\begin{array}{l}\text { Mutation of the TGA binding motifs of } \\
\text { the as-1-like element }\end{array}$ \\
\hline LS7 & Yes & Yes & TGA binding motif mutation $L S 7$ \\
\hline LS7-LS10 & No & Yes & $\begin{array}{l}\text { Sequence substitutions in } L S 7 \text { and } \\
L S 10\end{array}$ \\
\hline$P R-1$ & Yes & Yes & 1294bp full length PR-1 promoter \\
\hline PR-1 as-1 VAR & Yes & Yes & $\begin{array}{l}\text { The sequence of the } a s \text { - } 1 \text {-like element } \\
\text { linker region is changed to AAAA }\end{array}$ \\
\hline$P R-1$ linker in as -1 & No & Yes & $\begin{array}{l}\text { The TGA binding motifs of the as-1- } \\
\text { like element are substituted against the } \\
\text { binding motifs of CaMV35S as-1 }\end{array}$ \\
\hline$W 1$ & No & Yes & $\begin{array}{l}\text { Deletion of the first w-box downstream } \\
\text { of the as-1-like element }\end{array}$ \\
\hline$W 1-2$ & No & Yes & $\begin{array}{l}\text { Deletion of the first two w-boxes } \\
\text { downstream of the } a s \text { - } 1 \text {-like element }\end{array}$ \\
\hline$W 1-3$ & No & Yes & $\begin{array}{l}\text { Deletion of the whole w-box cluster } \\
\text { downstream of the } a s \text { - } 1 \text {-like element }\end{array}$ \\
\hline$W 2$ & No & Yes & $\begin{array}{l}\text { Deletion of the second w-box } \\
\text { downstream of the } a s \text { - } 1 \text {-like element }\end{array}$ \\
\hline
\end{tabular}

All plasmids were transformed in different plant genotypes via agrobacterium tumefaciens mediated gene transfer. A detailed list of transgenic plants generated with these plasmids is attached in the "supplemental data"-section of this work. 


\subsection{Plasmids for cloning- and transient assay purposes}

\begin{tabular}{|l|l|l|}
\hline Plasmid & \multicolumn{1}{|l|}{ Description } & Reference \\
\hline pHBT & $\begin{array}{l}\text { Vector for transient expression } \\
\text { in plant cells, pHBTL-sGFP } \\
\text { derivative, deletion of sGFP } \\
\text { gene by NcoI / NotI restriction, } \\
\text { Klenow fill in and religation, } \\
\text { amp }\end{array}$ & \\
\hline pSK-T & $\begin{array}{l}\text { Vector for cloning and and 2002) } \\
\text { sequencing, lacZa, amp }\end{array}$ & Kriete, unpublished \\
\hline
\end{tabular}

\subsubsection{Organisms}

\subsubsection{Bacteria}

\begin{tabular}{|c|c|c|}
\hline Species & Properties & Reference \\
\hline $\begin{array}{l}\text { Agrobacterium tumefaciens } \\
\text { GV3101 }\end{array}$ & $\begin{array}{l}\text { PMP90RK } \\
\text { rif }^{r}, g m^{r}\end{array}$ & (Koncz and Schell, 1986) \\
\hline Escherichia coli DB3.1 & $\begin{array}{l}\mathrm{F}^{-}, \quad g y r \mathrm{~A} 462, \quad \text { end } \mathrm{A} 1, \\
\mathrm{D}(\operatorname{sr} 1-r e c \mathrm{~A}), \quad m c r \mathrm{~B}, \quad m r r, \\
h s d \mathrm{~S} 20\left(\mathrm{r}_{\mathrm{B}}^{-} \mathrm{m}_{\mathrm{B}}^{-}\right), \sup \mathrm{E} 44, \\
\text { ara-14, galK2, lac } \mathrm{Y} 1, \\
\text { proA2, rps } \mathrm{L} 20\left(\mathrm{Sm}^{\mathrm{r}}\right), x y l-\mathrm{S}, \\
\lambda \text {-leu, } m t l-1\end{array}$ & (Bernard et al. 1993) \\
\hline Escherichia coli $\mathrm{DH} 5 \alpha$ & $\begin{array}{l}\mathrm{F}^{-}, \text {gyrA } 96 \text { (Nalr), recA1, } \\
\text { end } \mathrm{A} 1, \text { thi-1, hsd } \mathrm{R} 17 \text { (rk- } \\
\mathrm{mk}+), \ln \mathrm{V} 44, \text { deo } \mathrm{R}, \mathrm{D} \\
(\text { lacZYA-argF) } \mathrm{U} 169 \\
{[\mathrm{p} 80 \mathrm{dD}(\text { lacZ)M15] }}\end{array}$ & (Hanahan 1983) \\
\hline
\end{tabular}




\subsubsection{Plant genotypes}

\begin{tabular}{|c|c|c|}
\hline Genotype & Description & Reference \\
\hline Columbia, Col-0 & Wildtype & $\begin{array}{l}\text { NASC Stock Nr. N1092, } \\
\text { NASC } 2002\end{array}$ \\
\hline nprl-1 mutant & $\begin{array}{l}\text { Knock out line lacking } \\
\text { functional NPR1 }\end{array}$ & (Cao et al. 1994) \\
\hline snil-1 mutant & $\begin{array}{l}\text { Knock out line lacking } \\
\text { functional }\end{array}$ & (Li et al. 1999) \\
\hline snil-1/npr1-1 mutant & $\begin{array}{l}\text { snil-1 and nprl-1 double } \\
\text { mutant; Bgl2:GUS reporter }\end{array}$ & (Li et al. 1999) \\
\hline $\begin{array}{l}\operatorname{tga} 2 \operatorname{tga} 3 \text { tga5 tga6 } \\
\text { quadruple mutant }\end{array}$ & $\begin{array}{l}\text { Knock out line lacking all } \\
\text { three class II TGA } \\
\text { transcription factors and } \\
\text { TGA3, kindly provided by } \\
\text { X. Dong }\end{array}$ & (Kesarwani et al. 2007) \\
\hline tga6 mutant & $\begin{array}{l}\text { Single knock out of TGA6, } \\
\text { kindly provided by Y. } \\
\text { Zhang }\end{array}$ & (Zhang et al. 2003) \\
\hline $\operatorname{tga} 2 \operatorname{tg} a 5$ double mutant & $\begin{array}{l}\text { TGA2 and TGA5 double } \\
\text { mutant, kindly provided by } \\
\text { Y. Zhang }\end{array}$ & (Zhang et al. 2003) \\
\hline $\begin{array}{l}\operatorname{tga} 2 \text { tga5 tga6 triple } \\
\text { mutant } \\
\text { (tga2,5,6 mutant) }\end{array}$ & $\begin{array}{l}\text { Knock out line lacking all } \\
\text { three class II TGA } \\
\text { transcription factors, } \\
\text { impaired in SAR, kindly } \\
\text { provided by Y. Zhang }\end{array}$ & (Zhang et al. 2003) \\
\hline
\end{tabular}




\subsubsection{Growing media}

\begin{tabular}{|l|l|}
\hline Medium & Content \\
\hline $\begin{array}{l}\text { dYT medium } \\
\text { for bacteria }\end{array}$ & $20 \mathrm{~g} / \mathrm{L}$ Tryptone; $10 \mathrm{~g} / \mathrm{L}$ Yeast extract; $10 \mathrm{~g} / \mathrm{L} \mathrm{NaCl}$ \\
\hline $\begin{array}{l}\text { LB medium for } \\
\text { bacteria }\end{array}$ & $10 \mathrm{~g} / \mathrm{L}$ Tryptone; $5 \mathrm{~g} / \mathrm{L}$ Yeast extract; $10 \mathrm{~g} / \mathrm{L} \mathrm{NaCl}$ \\
\hline $\begin{array}{l}\text { MS medium for } \\
\text { plants }\end{array}$ & $\begin{array}{l}4.4 \mathrm{~g} / \mathrm{L} \text { MS medium; 1g/L MES; pH } 5.7 \text { with } \mathrm{KOH} ; 6,8 \mathrm{~g} / \mathrm{L} \text { select } \\
\text { agar }\end{array}$ \\
\hline
\end{tabular}

\subsubsection{Standard buffers}

\begin{tabular}{|c|c|}
\hline Buffer & Content \\
\hline PBS $(10 x)$ & $\begin{array}{l}\text { 1.4 M NaCl, } 27 \mathrm{mM} \mathrm{KCl}, 100 \mathrm{mM} \mathrm{Na} \mathrm{HPO}_{4}, 18 \mathrm{mM} \mathrm{KH} \mathrm{PO}_{4}, \mathrm{pH} \\
7.3\end{array}$ \\
\hline PBS-T (1 x) & 1 x PBS with $0.05 \%(\mathrm{v} / \mathrm{v})$ Tween-20 \\
\hline TAE $(20 x)$ & $0.8 \mathrm{M}$ Tris, $2.3 \%(\mathrm{v} / \mathrm{v})$ acetic acid, $20 \mathrm{mM}$ EDTA \\
\hline TE & $10 \mathrm{mM}$ Tris, $1 \mathrm{mM}$ EDTA, pH 7.5 \\
\hline Buffer O+ & $\begin{array}{l}50 \mathrm{mM} \text { Tris- } \mathrm{HCl}\left(\mathrm{pH} 7,5 \text { at } 37^{\circ} \mathrm{C}\right) ; 10 \mathrm{mM} \mathrm{MgCl}_{2} ; 100 \mathrm{mM} \mathrm{NaCl} \text {; } \\
0,1 \mathrm{mg} / \mathrm{ml} \mathrm{BSA}\end{array}$ \\
\hline Buffer B+ & $10 \mathrm{mM}$ Tris- $\mathrm{HCl}\left(\mathrm{pH} 7,5\right.$ at $\left.37^{\circ} \mathrm{C}\right) ; 10 \mathrm{mM} \mathrm{MgCl} 2 ; 0,1 \mathrm{mg} / \mathrm{ml} \mathrm{BSA}$ \\
\hline Buffer G+ & $\begin{array}{l}10 \mathrm{mM} \text { Tris- } \mathrm{HCl}\left(\mathrm{pH} 7,5 \text { at } 37^{\circ} \mathrm{C}\right) ; 10 \mathrm{mM} \mathrm{MgCl}_{2} ; 50 \mathrm{mM} \mathrm{NaCl} ; 0,1 \\
\mathrm{mg} / \mathrm{ml} \mathrm{BSA}\end{array}$ \\
\hline Buffer R+ & $\begin{array}{l}10 \mathrm{mM} \text { Tris- } \mathrm{HCl}\left(\mathrm{pH} 8,5 \text { at } 37^{\circ} \mathrm{C}\right) ; 10 \mathrm{mM} \mathrm{MgCl}_{2} ; 100 \mathrm{mM} \mathrm{KCl} ; 0,1 \\
\mathrm{mg} / \mathrm{ml} \mathrm{BSA}\end{array}$ \\
\hline Buffer Y+ & $\begin{array}{l}33 \mathrm{mM} \text { Tris-Acetat }\left(\mathrm{pH} 7,9 \text { at } 37^{\circ} \mathrm{C}\right) ; 10 \mathrm{mM} \text { magnesium acetat ; } 66 \\
\mathrm{mM} \text { potassium acetat; } 0,1 \mathrm{mg} / \mathrm{ml} \mathrm{BSA}\end{array}$ \\
\hline
\end{tabular}




\subsection{Methods}

\subsubsection{Standard molecular methods}

\subsubsection{Isolation of plasmid DNA from $E$. coli}

\subsection{Alkaline lysis}

Small amounts of plasmid DNA for analytical purposes were isolated from E. coli using a modification of the alkaline lysis method. E. coli overnight culture (stationary phase) of $1.5 \mathrm{~mL}$ was collected by centrifugation at $13000 \mathrm{rpm}$ for 1 minute. The supernatant was removed and the cells were resuspended in $100 \mu \mathrm{L}$ of buffer I for plasmid DNA isolation (50 mM Tris-HCl, $\mathrm{pH} 8.0 ; 10 \mathrm{mM}$ EDTA; $100 \mu \mathrm{g} / \mu \mathrm{L}$ RNase A). The cell suspension was lysed for 5 minutes on ice using $200 \mu \mathrm{L}$ of buffer II $(0.2 \mathrm{M} \mathrm{NaOH} ; 1 \%$ (w/v) SDS). The suspension was neutralized with $150 \mu \mathrm{L}$ of buffer III (29.4 g potassium acetate; $5 \mathrm{~mL}$ formic acid and water till $100 \mathrm{~mL}$ ). The solution was mixed well by inverting 6-8 times and the suspension was centrifuged for 10 minutes at $13000 \mathrm{rpm}$ at room temperature. The aqueous solution $(\sim 400 \mu \mathrm{L})$ was transferred into a new microcentrifuge tube containing $1 \mathrm{~mL}$ of $96 \%(\mathrm{v} / \mathrm{v})$ ethanol. The DNA was left to precipitate for $20 \mathrm{~min}$ at $-20^{\circ} \mathrm{C}$. Plasmid DNA was collected by centrifugation for 10 minutes at $13000 \mathrm{rpm}$ and $4^{\circ} \mathrm{C}$. The pellet was washed with $70 \%(\mathrm{v} / \mathrm{v})$ ethanol and airdried for 10 minutes at $37^{\circ} \mathrm{C}$. The DNA was dissolved in $20 \mu \mathrm{L}$ of EB buffer $(10 \mathrm{mM}$ Tris-HCl, $\mathrm{pH} 8.5)$.

\subsection{Isolation of high- quality plasmid DNA}

For sequencing and Gateway® cloning purposes, high-purity plasmid DNA was isolated using QIAprep (Qiagen) or Nucleospin Mini kit (Machery\&Nagel) following the manufacturer's instructions. Optional steps were always followed according to the manufacturer's recommendation. A $5 \mathrm{~mL}$ overnight culture was used to isolate plasmid and the isolated DNA was eluted with $50 \mu \mathrm{L}$ (high copy) or $30 \mu \mathrm{L}$ (low copy) EB buffer or water (ultra pure).

Larger amounts of plasmid DNA from E. coli with high purity were isolated using Qiagen or Macherey-Nagel Midi and Maxi kit depending upon the required end concentration. Manufacturer's protocol including the optional recommendations was followed and final elution volume depended on the plasmid copy number, size of the DNA pellet to be eluted and final concentration required. 


\subsubsection{Measurement of DNA- and RNA concentrations}

The concentration of nucleic acids was estimated by measuring their absorption in a spectrophotometer at a wavelength of $260 \mathrm{~nm}$ (maximum nucleic acid absorption value; due to the $\pi$-electron systems of the heterocycles of the nucleotides). In a cuvette having $10 \mathrm{~mm}$ path-length where $\mathrm{OD}_{260}$ reading is 1 corresponds to 50 and $40 \mu \mathrm{g} / \mathrm{mL}$ doublestranded DNA and RNA,respectively. Absorption at $280 \mathrm{~nm}$ (for the presence of aromatic rings from amino acids and phenol compounds) was used to give information about the purity of the DNA or RNA sample, where an optimal ratio $\mathrm{OD}_{260} / \mathrm{OD}_{280}$ is in the range of 1.9-2.0 for RNA and 1.8 for DNA. DNA concentrations lower than $100 \mathrm{ng} /$ $\mu \mathrm{L}$ were measured on an agarose gel using the Gene Ruler Ladder Mix.

\subsubsection{Separation of DNA on agarose gels}

The electrophoretic separation of DNA for analytical and preparative purpose was done in a horizontal agarose gel $(10 \mathrm{~cm} \times 7 \mathrm{~cm} \times 0.3 \mathrm{~cm}, 16$ lanes $)$ with $1 \times$ TAE as running buffer. DNA fragments ranging between $500 \mathrm{bp}$ and $14 \mathrm{~kb}$ were run in an agarose gel concentration of $1 \%$ where DNA fragments with lower size were run in a $2 \%$ agarose gel. DNA samples were mixed with $1 / 10$ volume of $10 x$ DNA loading buffer, loaded in separate lanes and run at $120 \mathrm{~V}$ for $40-45 \mathrm{~min}$. Ethidiumbromide solution $(0.1 \% \mathrm{w} / \mathrm{v})$ was used to stain the DNA fragments. The detection of DNA was done under UV light $(260 \mathrm{~nm})$. When a preparative gel was run and particular band fragments were needed to cut out, detection was done using larger wavelength UV light $(320 \mathrm{~nm})$. Before exposure to the UV light, the gel was rinsed briefly in $\mathrm{H}_{2} \mathrm{O}$ to reduce background staining. In a gel-documentation station, gels were visualized on a UV-transilluminator and documented. The sizes and amount of the DNA fragments were determined using DNA standards.

The elution of DNA fragments from agarose gel was done using the QIAquick or Nucleospin Extract II Gel Extraction kit following the manufacturer's instructions. The eluted fragments were verified by electrophoresis as described above.

\subsubsection{Separation of DNA on polyacrylamid gels (PAA)}

The use of PAA-gels enables an accurate separation of small DNA fragments (1$300 \mathrm{~kb})$. In this work, vertical $5 \%$ PAA-gels $(17 \mathrm{~cm} \mathrm{x} 15,5 \mathrm{~cm} \times 0,15 \mathrm{~cm}, 11$ wells) were utilized for preparation of radioactively labeled EMSA fragments (3.2.1.9.8) and gel retardation experiments (3.2.1.9.9). Each gel $(50 \mathrm{ml})$ consisted of: $5 \%(\mathrm{w} / \mathrm{v})$ acrylamid:N,N'-methylenbisacrylamid (19:1), $10 \%(\mathrm{v} / \mathrm{v})$ glycerin and $1 \mathrm{x}$ TBE $(5 \mathrm{x}$ TBE: 0,5M tris; 0,5M boric acid; 10mM EDTA), supplemented with 0,04\% (v/v) TEMED and $0,06 \%(\mathrm{w} / \mathrm{v})$ APS. Polymerization of the gel took place under $4^{\circ} \mathrm{C}$ over 5 hours. The electrophoresis was performed in $1 \mathrm{x}$ TBE buffer at $60-150 \mathrm{~V}$ and $4^{\circ} \mathrm{C}$. As markers, bromphenolblue and xylencyanol FF were used, running on the heights of 35bp- and 130bp DNA fragments, respectively. 


\subsubsection{Restriction digestion of DNA}

Type II endonucleases were used to digest a double stranded DNA molecule for analytical and cloning purposes. The enzymes cut the DNA either as 5' or 3' "sticky" overhangs or as blunt ends. The digestion reactions were incubated in a buffer system optimized for the used enzyme and in the case of double digestion a universal buffer system was used. The activity of the restriction enzymes was estimated in "units" (U), where $1 \mathrm{U}$ was defined as that amount of enzyme cutting completely $1 \mu \mathrm{g}$ of $\lambda$ DNA in 60 minutes at optimal conditions. The minimal amount of enzyme necessary for each restriction was determined according to the following formula:

$\mathrm{U}=(\mathrm{bp}[\lambda] \mathrm{x}$ No. of restriction sites in target DNA) / (No. of restriction sites in $[\lambda] \mathrm{x} b \mathrm{p}$ of target DNA) with $\lambda=48500 \mathrm{bp}$

The incubation temperature was $37^{\circ} \mathrm{C}$ unless otherwise mentioned for particular restriction enzyme. Due to the adverse effect of high glycerol concentration, the total volume of restriction enzymes should not extend more than $10 \%$ in the restriction mix.

\subsubsection{Ligation of DNA fragments}

The conventional cloning of a DNA fragment into a selected plasmid was performed using the T4-DNA ligase enzyme, which is able to catalyze the formation of a phosphodiesther chemical bond between free 5'-phosphate and 3'-OH groups of double stranded DNA fragments and vectors. The donor DNA fragment (10x accesses over the vector) was incubated with the vector DNA, $2 \mu \mathrm{L}$ of ligation buffer and $1 \mu \mathrm{L}$ of T4DNA ligase for 2 hours at room temperature. The ligation of DNA fragments with blunt ends was performed in the presence of $5 \%$ (w/v) PEG 4000 with the ligation mix described above. Ligase activity was destroyed by heating at $65^{\circ} \mathrm{C}$ for 10 min before using the ligated DNA for transformation.

\subsubsection{Gateway ${ }^{\circledR}$ cloning}

The cloning of binary vectors for transient and stable plant transformation purposes has been performed with the gateway® cloning system from Invitrogen. The gateway® technology is based on the site specific recombination of bacteriophage lambda and thereby provides a fast method to move DNA sequences between multiple vector systems without the use of restriction enzymes (Landy 1989; Hartley et al. 2000). All cloning steps have been performed as mentioned in the Invitrogen manual, Version E, 22. September 2003. 


\subsubsection{Sequencing of DNA}

The DNA sequencing was done using the BigDye Terminator RR Mix Cycle Sequencing kit. The principle of DNA sequencing is based on the chain-termination method (Sanger et al., 1977). In the chain-termination method, dideoxynucleotides (terminators) are incorporated into a newly synthesized complementary chain that will lead to stop its elongation in a PCR reaction. Each of dideoxynucleotides is labeled with a specific fluorescent dye and the terminated chains can be specifically detected using an ABI Prism 3100 Capillary Sequencer (Applied Biosystems). The PCR sequencing reaction was performed using 500-1000 ng plasmid DNA, 5 pmol primer, $2 \mu \mathrm{L}$ RR mix (ready reaction) and $\mathrm{H}_{2} \mathrm{O}$ up to $10 \mu \mathrm{L}$. The samples were subjected to 25 cycles of: 10 seconds at $95^{\circ} \mathrm{C}, 5$ seconds at $50^{\circ} \mathrm{C}, 4$ minutes at $60^{\circ} \mathrm{C}$ in a thermocycler. The DNA product was precipitated using $9.5 \mu \mathrm{L}$ water and $30.5 \mu \mathrm{L}$ of absolute ethanol and left for 1 hour. The DNA was collected by centrifugation for 20 minutes at $13000 \mathrm{rpm}$. The pellet was washed using $125 \mu \mathrm{L} 70 \%$ ethanol and then centrifuged for 10 minutes at $13000 \mathrm{rpm}$. The pellet was dried at $95^{\circ} \mathrm{C}$ for one minute and resuspended in $10 \mu \mathrm{L}$ of HiDi-reagent. The samples were placed on ice. The reactions were loaded on an ABIPrism 3100 capillary electrophoresis sequencing station for analysis.

\subsubsection{Cloning of vectors}

\subsection{Hybridization of complementary DNA fragments}

1nmole of complementary DNA oligonucleotides were added in a total volume of $20 \mu 1$ (screw cap reaction tube) and heated for $10 \mathrm{~min}$ in a $100^{\circ} \mathrm{C}$ water bath. To assure a smooth hybridization, the samples were cooled down to room temperature in the water bath over night.

\subsection{Cloning of DNA fragments for EMSA studies}

The fragments for EMSAs were obtained by hybridization of complementary oligonucleotides. All oligonucleotides contained open restriction sites (HindIII at the $5^{5}$ end and EcoRI at the $3^{\prime}$ end) for ligation in the pUC18 or pSK Vectors and BpiI restriction sites for generation of polyT overhangs and subsequent radioactive labeling.

\subsection{1 as-1}

77bp fragment inserted in pUC18-as-1 (Thurow et al. 2005)

HindIII -

gcatgctgcaggtcgactctagaTGACGTAAgggaTGACGCACtctagaggatccecgggtaccgagctc - EcoRI

The TGA recruitment sites within the as- 1 element are labeled by bold capital letters. 


\subsection{LS4-LS8}

78 bp fragment inserted in pSK-LS4-LS8

HindIII - ttgcGTCTTCtctattgactgtttctctacgtcactattttacttacgtcatagatgtggcggcatGAA

GACgcaaaa - EcoRI

The bold capital letters show the BpiI restriction sites while the bold letters in the center of the fragment show the TGA binding sites of the as-1-like element.

\subsection{LS5-LS10}

84bp fragment inserted in pSK-LS5-LS10

HindIII - ttgcGTCTTCtctacgtcactattttacttacgtcatagatgtggcggcatatattcttcaggacttttcag GAAGACgcaaaa - EcoRI

The capital letters show the BpiI restriction sites while the bold letters in the center of the fragment show the TGA binding sites of the as-1- like element.

\subsection{4 w1+w2+w3}

$89 \mathrm{bp}$ fragment containing the whole w-box cluster downstream of the as-1-like element inserted in pSK- $W 1+W 2+W 3$..

HindIII - ttgcGTCTTCcttgactttttttcttttatttgaaaattgactgtagatataaacttttattttttctgactgtaaa

GAAGACgcaaaa - EcoRI

The bold capital letters show the BpiI restriction sites while the bold labeled letters in the center of the fragment show the putative w-boxes.

\subsection{Cloning of full length $P R-1$ promoter constructs}

All full length $P R-1$ promoter derivates shown in this work were constructed as translational fusions reaching from $+34 \mathrm{bp}$ to $-1294 \mathrm{bp}$ relative to the transcriptional start site. The original fragment was amplified from Col-0 gDNA using the iProof TM highfidelity PCR kit from Bio-Rad. For amplification, the primers PR-1-1293-F-SalI and PR-1-5 UTR-pBGWFS7 were used considering the standard protocol and PCR cycler program from the manual. The substitution and deletion constructs were obtained via overlap extension PCR (OE-PCR). Therefore, the two mentioned primers were utilized seperately in two iProof ${ }^{\mathrm{TM}}$ PCRs in combination with primers carrying the mutation of interest (see primer list in 2.1.8.1). The two different fragments yieded by the first PCR were purified after separation on a 1\% agarose gel and diluted 1:200. The two diluted fragments were used as templates in a second PCR, again employing the primers $P R-1$ 1293-F-SalI and PR-1-5 UTR-pBGWFS7. The resulting PCR fragment contained the desired DNA modification and was digested subsequently with restriction endonucleases to ligate the fragment in pUC18-Entry2-PR-1. If the mutation was located within the linker scanning region described by Lebel et al., Mph1103I and Eco91I were chosen for cloning, while w-box deletion fragments located downstream of this sector were digested with Mph1103I and BpiI prior to ligation. The ligated vectors have been transformed in E. coli to accumulate plasmid DNA. All plasmids 
were sequenced, at least in the region of the substituted DNA fragment, to exclude the possibility of randomly created sequence mutations due to PCR mistakes.

\subsection{Cloning of $P R-1$ promoter deletion constructs}

The $P R-1$ promoter construct lacking $495 \mathrm{bp}$ between $-74 \mathrm{bp}$ and $-569 \mathrm{bp}$ has been cloned in several steps. The initial promoter sequence for this derivate was not the full length promoter described in 3.2.1.9.3, but a promoter amplified from Col- 0 gDNA with the primer combination $P R-1-1293-\mathrm{F}-$ SalI and $P R-1-1-\mathrm{R}$. This means, that the 5 UTR of $P R-1$ is missing in this sequence. Furthermore, a fake sequence has been inserted by substitution of region $-1294 \mathrm{bp}$ to $-569 \mathrm{bp}$ against a stretch of coding sequence derived from the CAT gene using the restriction sites SalI and Eco91I. For PCR mediated amplification of the CAT gene, the primer combination $C A T 483 F$ / CAT483R has been used on the template plasmid pTAX-CAT. The deletion between -569 and -68 was created by ligation of the vector fragment pUC18-Entry2-CAT-PR-1 cut with Eco91I (816) and HpaI (-68), and the promoter fragments from Eco91I (-816) to $N d e \mathrm{I}_{\text {(fill in) }}(-$ 573). As the fill in reaction of the NdeI overhanging ends had destroyed the Mph1103I restriction site an overlap extension PCR was performed to obtain a $P R-1$ deletion construct that is more versatile concerning cloning purposes due to restoration of this restriction site. The primers used for this modification were $P R-13{ }^{`} D-F$ and $P R-13{ }^{`} D$ $\mathrm{R}$ on the pUC18-Entry2-3 $D$ plasmid as template.

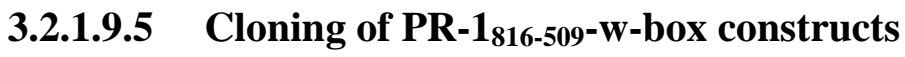

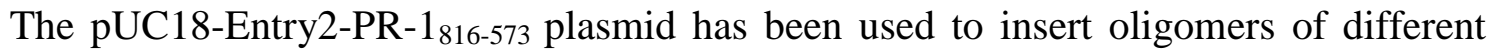
sizes in the Mph1103I restriction site. These oligomeres contain no w-box ( $3^{`} \mathrm{D}$ oligo Chip-R), one w-box ( $3^{`} \mathrm{D}$ oligo ChIP-R + w1), two w-boxes ( $3^{`} \mathrm{D}$ oligo ChIP-R + w1 $+\mathrm{w} 2$ ) or all three w-boxes downstream of the $a s-1$-like element ( $3^{`} \mathrm{D}$ ologo ChIP-R $+\mathrm{w} 1$ $+\mathrm{w} 2+\mathrm{w} 3)$ of the $P R-1$ promoter.

\subsection{Klenow fragment-mediated filling of 5 'end overhangs}

The klenow fragment of $E$. coli DNA polymerase I shows $5 \rightarrow 3$ polymerase- and $3 \backslash \rightarrow$ 5 exonuclease activity. The enzyme is frequently used to fill up DNA fragments with "sticky" 5 - overhangs or to digest fragments with 3 - overhangs to obtain blunt ended DNA fragments. The klenow fragment is active in all restriction buffers mentioned in the standard buffer table (2.1.11).

Procedure for $3 \mu \mathrm{g}$ of digested DNA in a volume of $30 \mu \mathrm{l}$ :

0,33mM dATPs, dCTPs, dGTPs and dTTps 2U klenow fragment; 1x restriction buffer. The reaction was incubated for $1 \mathrm{~h}$ at RT and subsequently heated to $75^{\circ} \mathrm{C}$ for 10 minutes to inactivate the enzyme. A following gel elution yielded the cleaned up DNA fragment. 


\subsection{T4 polymerase-mediated filling of 5 'end overhangs}

T4 is a bacteriophage of $E$. coli. The activities of T4 DNA polymerase are very similar to Klenow fragment of DNA polymerase I - it functions as a $5^{\prime} \rightarrow 3^{`}$ DNA polymerase and a $3^{\prime} \rightarrow 5^{\prime}$ exonuclease, but does not have $5^{\prime} \rightarrow 3^{\prime}$ exonuclease activity. The advantage of the T4 polymerase is the 200 times higher efficiency of the $3 \rightarrow 5^{`}$ exonuclease activity in comparison to Klenow fragment, making it a preferred choice for blunting DNAs with 3'overhangs. $1 \mu \mathrm{g}$ of cleaned up DNA fragments were supplemented with $5 \mathrm{x}$ reaction buffer for $\mathrm{T} 4$ polymerase, $0,1 \mathrm{mM}$ of each dNTP, 1U T4 DNA polymerase and water to a final volume of $20 \mu 1$. The mixture was incubated for 5 minutes at RT, while a subsequent 10 minutes heating step at $75^{\circ} \mathrm{C}$ inactivated the enzyme. After gel elution, the modified fragments were used for further cloning purposes.

\subsection{Radioactive labeling of EMSA fragments}

The radioactive labeling of EMSA fragments with $\alpha-{ }^{32} \mathrm{P}-\mathrm{dATP}$ has been achieved by filling up 5 overhangs of restricted vectors utilizing klenow fragment as described under 3.2.1.9.6. The depletion of non-integrated $\alpha-{ }^{32} \mathrm{P}-\mathrm{dATPs}$ from the mixture has subsequently been performed via gelfiltration using Micro Spin ${ }^{\mathrm{TM}}$ G25 columns from Pharmacia and the appropriate protocol. Afterwards, the labeled fragment was separated from the labeled vector by PAA-gel electrophoresis (3.2.1.4), cut out and solved in $800 \mu \mathrm{l}$ EB buffer. Detection of the radioactive fragments was realized by autoradiography.

\subsection{Electrophoretic mobility shift assays (EMSAs)}

The binding reaction occurred in an overall volume of $30 \mu 1$ in $1 \mathrm{x}$ binding buffer $(5 \mathrm{x}$ binding buffer: $125 \mathrm{mM}$ HEPES-KOH, $\mathrm{pH} 7,6 ; 50 \mathrm{mM} \mathrm{MgCl}_{2} ; 1 \mathrm{mM} \mathrm{CaCl}_{2} ; 5 \mathrm{mM}$ DTT; $2 \mathrm{mM}$ PMSF; $50 \%(\mathrm{v} / \mathrm{v})$ glycerin). $3 \mu \mathrm{g}$ of PolydI/dC has been added to the protein samples $(10 \mu \mathrm{g})$ to avoid unspecific DNA binding and the extracts were pre-incubated on ice for 10 minutes. In case of supershift assays, $1 \mu 1$ of $\alpha$ TGA2/5 serum or the corresponding pre-immune serum were supplemented. After $4 \mu l$ of the labeled fragment has been added, there was a further 10 minutes incubation step at RT. The samples were subsequently mixed with $10 \mu 1$ loading buffer $(50 \%(\mathrm{v} / \mathrm{v})$ glycerin; $42 \%(\mathrm{v} / \mathrm{v}) 5 \mathrm{x}$ binding buffer) and transferred in the wells of a 5\% PAA-gel (3.2.1.4). Before loading, the gel had to run for at least $1 \mathrm{~h}$ at $150 \mathrm{~V}$ to assure a better quality of the gel. The loaded gel has been running over night at $65 \mathrm{~V}$ and $4^{\circ} \mathrm{C}$. On the next day, the gel was positioned on $3 \mathrm{MM}$ blotting paper and wrapped in plastic foil. Drying of the gel has been performed on an $80^{\circ} \mathrm{C}$ vacuum gel drying device over a period of $2 \mathrm{~h}$. For exposition, the gel was placed in a cassette together with an IP-screen. After $4 \mathrm{~h}$, the signal intensities could be detected using a bioimager (BAS-1000 from Fuji). The images were analyzed with the programs PCBAS ${ }^{2} .09$ and TINA $® 2.0$ from Raytest. 


\subsubsection{Gene transfer to $E$. coli}

E. coli cells have no competent nature, i.e. they are not able to accept foreign DNA molecules from the environment. To enable the bacterial cells to take up circular vector DNA they have to be made competent using special treatments. Two transformation methods were used to transform competent bacteria cells: The heat shock and the electroporation. The heat shock method was used only to transform E. coli chemical competent cells (Hanahan, 1983). In brief, $200 \mu \mathrm{L}$ competent $E$. coli cells were thawed on ice for $20 \mathrm{~min}, 50 \mathrm{ng}$ of plasmid DNA was added to the cells and mixed gently. The mixture was incubated on ice for 30 minutes. The cells were heat shocked for $90 \mathrm{~s}$ at $42^{\circ} \mathrm{C}$. $700 \mu \mathrm{L}$ of LB medium were added to the tube and the suspension was mixed on a roller for $45-60 \mathrm{~min}$ at $37^{\circ} \mathrm{C}$ depending on selectable antibiotic resistance marker. Different volumes of the culture were plated on plates containing LB medium supplemented with antibiotics. The plates were incubated overnight at $37^{\circ} \mathrm{C}$.

The transformation using electroporation was done for $E$. coli cells according to a slight modification of (Dower et al., 1988). The electroporation was done using a Gene Pulser II. Bacterial competent cells were thawed on ice slowly before adding $2 \mu \mathrm{L}$ of plasmid DNA. The mixture was transferred into an ice-cooled electroporation cuvette $(2 \mathrm{~mm}$ electrode distance). The cuvette was subjected to electroporation at $25 \mu \mathrm{F}, 2.5 \mathrm{kV}, 200$ $\Omega$. The cells were suspended immediately with $1 \mathrm{~mL} \mathrm{LB}$ medium and incubated for 45 $60 \mathrm{~min}$ at $37^{\circ} \mathrm{C}$. Different volumes of the culture were plated on LB media supplemented with antibiotics and incubated overnight (12-16 hours) at $37^{\circ} \mathrm{C}$.

\subsubsection{Gene transfer to A. tumefaciens}

Competent cells of Agrobacterium tumefaciens GV3101 were transformed by electroporation method. Cells were thawed on ice, mixed with respective plasmid DNA and transferred to an electroporation cuvette. Electric pulse $(2.5 \mathrm{kV}, 25 \mu \mathrm{F}, 400 \Omega)$ was applied for $\sim 5 \mathrm{~s}$. Subsequently, cells were incubated with $1 \mathrm{~mL} \mathrm{LB}$ medium for $2 \mathrm{~h}$ at $30^{\circ} \mathrm{C}$ and spread on selective YEB-plates. Incubation of plates was performed for 2-3 days at $30^{\circ} \mathrm{C}$. Transformed cells from plates were grown in $25 \mathrm{~mL}$ selective YEB liquid medium $\mathrm{o} / \mathrm{n}$ at $30^{\circ} \mathrm{C}$. From $5 \mathrm{~mL}$ of this pre-culture plasmid-DNA was extracted (QIAprep kit) to control the transformed cells. The rest of the pre-culture was transferred into $400 \mathrm{~mL}$ selective YEB liquid medium and incubated o/n at $30^{\circ} \mathrm{C}$. Cells were harvested by centrifugation $(2500 \mathrm{rpm}, 30 \mathrm{~min})$ and resolved in $5 \%$ sucrose solution to an $\mathrm{OD}_{600}$ of 0.8 . Silvet-L77 $(0.05 \%)$ was added to this solution prior to $A$. thaliana transformation. 


\subsubsection{Agrobacterium- mediated gene transfer to $A$. thaliana}

Transformation of A. thaliana with Agrobacterium was performed after (Clough 2005). Therefore, flowering plants were dipped into an Agrobacterium solution $(\mathrm{OD}=0,8)$. Plants were subsequently cultured to seed development. Selection was performed with respective selection markers on the integrated transgenic DNA.

\subsubsection{Plant growth conditions}

\subsection{Growth of transgenic reporter gene lines}

Approximately $200 \mathrm{~F} 2$ seeds of selected reporter gene lines were surface sterilized and sown on sterile $0 \mathrm{x}$ MS-plates comprising 0,5g/L MES. The control plates contained $15 \mu 1 / \mathrm{L}$ DMSO, while the plates used for induction treatment were supplemented with $30 \mu \mathrm{M}$ INA. The plants were grown for 18 days at $22^{\circ} \mathrm{C}$ under long day conditions $(14 \mathrm{~h}$ light/ 10h dark) and 60\% humidity.

\subsection{Plant growth conditions for transient expression studies}

Plants for protoplast transformation were grown on steamed soil under long day- $(16 \mathrm{~h}$ light/ $8 \mathrm{~h}$ dark) and weak light conditions over a period of three weeks at $22^{\circ} \mathrm{C}$ and $60 \%$ humidity. For each pot three plants were sown out.

\subsection{Plant growth conditions for EMSA studies}

Surface sterilized seeds were sown on sterile 0x MS- plates containing 0,5g/L MES and grown for 14 days under long day conditions ( $14 \mathrm{~h}$ light/ $10 \mathrm{~h}$ dark) at $22^{\circ} \mathrm{C}$. On day 13 , some of the plates were sprayed with $1 \mathrm{mM}$ SA. After $24 \mathrm{~h}$ of induction treatments, $100 \mathrm{mg}$ plant material was harvested for preparation of protein extracts. Alternatively, 0x MS- plates supplemented with $30 \mu \mathrm{M}$ INA were used to trigger chemically induced SAR. 


\subsubsection{RNA extraction}

The extraction method based on TRIZOL extraction can be used to extract RNA, DNA and proteins from plants (Chomczynski, 1993). This method uses a Phenol/ Chloroform (dichloromethane) extraction to solve RNA in the aqueous phase while other parts like chlorophyll is solved in the hydrophobic chloroform phase. The two thiocyanates in the extraction buffer inhibit RNAses. After grinding of the plant material under liquid nitrogen $1 \mathrm{~mL}$ extraction buffer was added to $\sim 150 \mathrm{mg}$ plant material. After shaking for $15 \mathrm{~min}$ at RT, chloroform $(200 \mu \mathrm{L})$ was added to each sample. After an additional shaking step (15 min, RT) and centrifugation $\left(12000 \mathrm{rpm}, 35 \mathrm{~min}, 4^{\circ} \mathrm{C}\right)$ the supernatant $(700 \mu \mathrm{L})$ was transferred to new microcentrifuge tubes. Precipitation buffer (HSPB) and 2-propanol (each $250 \mu \mathrm{L}$ ) were added and the samples were incubated for $10 \mathrm{~min}$ at RT and centrifuged $\left(12000 \mathrm{rpm}, 20 \mathrm{~min}, 4^{\circ} \mathrm{C}\right)$. After removing the supernatant, samples were dried at RT. The dried pellets were resolved in $50-100 \mu \mathrm{L}$ water (ultra pure). Concentration was measured as described in 2.2.1.2.

\subsubsection{Quantitative Realtime RT-PCR (qRT-PCR)}

RNA extraction of plant leaf material was performed as described above. DNaseI restriction was done before cDNA synthesis. $1 \mu \mathrm{g}$ of RNA, $1 \mu \mathrm{L}$ of $10 \mathrm{x}$ reaction buffer with $\mathrm{MgCl}_{2}$ (Fermentas, St. Leon-Roth, Germany) and desoxyribonuclease I (DNaseI), RNase-free was added with water to a final reaction volume of $10 \mu \mathrm{L}$. The mixture was incubated at $37^{\circ} \mathrm{C}$ for 30 minutes. To denatured the DNaseI $1 \mu \mathrm{L} 25 \mathrm{mM}$ EDTA was added and incubated at $65^{\circ} \mathrm{C}$ for 10 minutes. cDNA synthesis was performed with $1 \mu \mathrm{g}$ total RNA (DNA-free), 20 pmol of oligo-dT primer and 200 pmol of random nonamer oligonucleotides. Water was added to a final reaction volume of $12.5 \mu \mathrm{L}$. The mixture was heated to $70^{\circ} \mathrm{C}$ for $10 \mathrm{~min}, 20 \mathrm{nmol} \mathrm{dNTPs}, 4 \mu \mathrm{L} 5 \mathrm{x}$ reaction buffer (Fermentas, St. Leon-Roth, Germany) and 30 u ribonuclease inhibitor (Eppendorf, Hamburg, Germany) were added and the mixture was heated to $37^{\circ} \mathrm{C}$ for $10 \mathrm{~min}$. $100 \mathrm{u}$ of RevertAid ${ }^{\mathrm{TM}} \mathrm{H}$ Minus M-MuLV reverse transcriptase (Fermentas, St. Leon-Roth, Germany) was added (final volume $20 \mu \mathrm{L}$ ) and the mixture was incubated at $42^{\circ} \mathrm{C}$ for $70 \mathrm{~min}$, then heated to $70^{\circ} \mathrm{C}$ for $10 \mathrm{~min}$. The iCycler System (Bio Rad, Hercules, CA, USA) was used for the amplification and quantification of cDNA using QuantiTect Primer Assays (Qiagen, //www 1.qiagen.com/Products/Pcr/QuantiTect/PrimerAssays.aspx) for the respective genes and for ubiquitin 5 (UBQ5) as reference gene. The amplification mix consisted of $1 \mathrm{x} \mathrm{NH}_{4}$-reaction buffer (Bioline, Luckenwalde, Germany); $2 \mathrm{mM} \mathrm{MgCl}_{2} ; 100 \mu \mathrm{M}$ of dNTPs; $0.4 \mu \mathrm{M}$ of primers, $0.25 \mathrm{u}$ BIOTaq DNA polymerase (Bioline Luckenwalde, Germany); 10 nM Fluoresceine (BioRad, Hercules, CA, USA); 100,000 times diluted SYBR Green I solution (Cambrex, Rockland, ME, USA); $1 \mu \mathrm{L}$ of a 1:10 dilution of cDNA as template and water (ultra pure) added to a total volume of $25 \mu \mathrm{L}$. PCR consisted of a 6 min initial denaturation step at $95^{\circ} \mathrm{C}$ followed by 40 cycles of $20 \mathrm{~s}$ at $95^{\circ} \mathrm{C}, 20 \mathrm{~s}$ at $55^{\circ} \mathrm{C}$ and $40 \mathrm{~s}$ at $72^{\circ} \mathrm{C}$. 


\subsubsection{Preparation of protein extracts for LUC assays}

Protein extractions for luciferase activity assays have been prepared by application of $700 \mu \mathrm{l} 1 \mathrm{x}$ cell culture lysis reagent (Promega) to approximately $300-500 \mathrm{mg}$ of grinded and frozen plant material. The samples were shaken in an Eppendorf mixer until the plant powder was completely solved in the buffer. Afterwards the samples were placed on ice immediately. After centrifugation (10 min, $12.000 \mathrm{rpm}$ at $\left.4^{\circ} \mathrm{C}\right) 10 \mu \mathrm{l}$ of protein extract could be added in each well of a pre- cooled (ice bath) Greiner lumitrac 200 plate. The plate was subsequently covered under a hood (20 min; ice bath) to avoid background illumination during the LUC activity measurement. The LUC assays were started 5-10 min after insertion of the plate in the FLUOstar ${ }^{\circledR}$ Ultima luminescence plate reader. It is important to note that the samples should be used immidiately after preparation, because they cannot be stored over night without a severe decrease in LUC activity.

\subsubsection{Preparation of protein extracts for MUG assays}

Extraction of proteins for MUG assay purposes was conducted by addition of $700 \mu 1$ GUS extraction buffer $\mathrm{pH} 7.5$ (supplemented with $0,05 \%$ B-mercaptoethanol) to grinded plant material (300 - 500mg). After solvation of the powder, the samples were centrifuged $\left(10 \mathrm{~min}, 12.000 \mathrm{rpm}\right.$ at $\left.4^{\circ} \mathrm{C}\right)$ and the supernatant was transfered to a new 1.5 $\mathrm{ml}$ reaction tube. The samples could be stored at $-80^{\circ} \mathrm{C}$.

\subsubsection{Determination of protein concentrations}

Protein concentrations were estimated by two different methods. A colorimetric assay was used to determine the concentration from proteins extracted without detergent usage (for MUG assay purposes) according to (Bradford and Williams, 1976). The assay was conducted by pipetting equal amounts of protein extract into a microtiter plate containing $200 \mu \mathrm{L}$ of 5 -fold diluted Bradford reagent. The OD595 was measured with a MRX plate reader (Dynex). Protein concentrations were calculated with the help of a standard curve derived from different BSA protein amounts $(1,3$ and $6 \mu \mathrm{g})$ on the same plate. Proteins isolated from LUC reporter gene plants were measured with the BCA protein assay kit (Thermo scientific) according to the instruction manual.

\subsubsection{Measurement of RLU using the FLUOstar ${ }^{\circledR}$ Ultima platereader}

For the detection of relative light units emitted by protein extracts of transgenic reporter gene lines, the FLUOstar ${ }^{\circledR}$ platereader was used. All measurements have been performed under the same conditions, using a detection interval of $10 \mathrm{~s}$ and a relative gain value of 3600. The volume of 50 $\mu$ l luciferase substrate buffer (Promega) was applied to each well via one of the pumps integrated in the platereader. After detection of the emitted light, the values were calculated considering the protein concentrations of the extracts to obtain the RLU. 


\subsubsection{Measurement of relative GUS activities}

For the determination of GUS activities from protein extracts, $1 \mu l$ of a 1:100 dilution was employed in a MUG assay using a flat bottomed multititerplate. After addition of $99 \mu 1$ of MUG extraction buffer pH7,5 + $\beta$-mercaptoethanol, 100 $\mu 1$ of MUG-solution were added to each well and the plate was placed in a $37^{\circ} \mathrm{C}$ incubator to start the enzymatic reaction. 10 minutes later, a $t_{0}$ value was taken by pipetting $100 \mu l$ of the sample to $100 \mu \mathrm{l}$ of GUS-stop buffer $\left(200 \mathrm{mM} \mathrm{Na} \mathrm{CO}_{3}\right)$. The remaining reaction was stopped 60 minutes later to gain a $t_{60}$ value. The $t_{0}$ and $t_{60}$ values were used to calculate the specific GUS activities of the samples.

\subsubsection{Transient expression analysis by protoplast transformation}

Protoplast isolation and transformation was performed according to the method described by (Sheen 2001). For $P R-1$ promoter activity measurements, $20 \mu \mathrm{g}$ of promoter:GUS plasmids (pBGWFS7) were transformed in Arabidopsis protoplasts. For standardization $3 \mu \mathrm{g}$ of 35S:NAN plasmid was used (Kirby and Kavanagh 2002), alternatively, the protein concentrations were measured by colorimetric Bradford assays. GUS and NAN enzyme assays were performed according to (Kirby and Kavanagh 2002).

\subsubsection{Buffers used for protoplast transformations}

\begin{tabular}{|c|c|}
\hline Buffer & Content \\
\hline Enzyme solution & $\begin{array}{l}1-1,5 \% \text { cellulase } \mathrm{R} 10 \\
0,2-0,4 \% \text { macerozyme } \mathrm{R} 100,4 \mathrm{M} \text { mannitol } \\
20 \mathrm{mM} \mathrm{KCl} ; 20 \mathrm{mM} \mathrm{MES}, \mathrm{pH} 5,7 \\
\text { Heat the enzyme solution at } 55^{\circ} \mathrm{C} \text { for } 10 \mathrm{~min} \text { (to } \\
\text { inactivate proteases and enhance enzyme } \\
\text { solubility), The enzyme solution is light brown but } \\
\text { clear (passed through a } 0,45 \mu \mathrm{m} \text { filter) }\end{array}$ \\
\hline PEG solution $(40 \%, \mathrm{v} / \mathrm{v})$ & $\begin{array}{l}\text { 4g PEG4000; } 3 \mathrm{ml} \mathrm{H}_{2} \mathrm{O} ; 2,5 \mathrm{ml} \mathrm{0,8} \mathrm{M} \mathrm{mannitol} \\
1 \mathrm{ml} 1 \mathrm{M} \mathrm{Ca}\left(\mathrm{NO}_{3}\right)_{2} \text { or } \mathrm{CaCl}_{2}\end{array}$ \\
\hline $\begin{array}{l}\text { Washing and incubation } \\
\text { solution (WI) }\end{array}$ & 0,5M mannitol ; 4mM MES, pH 5,7 ; 20mM KCl \\
\hline W5 solution & $\begin{array}{l}154 \mathrm{mM} \mathrm{NaCl} ; 125 \mathrm{mM} \mathrm{CaCl}_{2} ; 5 \mathrm{mM} \mathrm{KCl} \\
2 \mathrm{mM} \mathrm{MES} \mathrm{(pH} \mathrm{5,7)}\end{array}$ \\
\hline MMg solution & $0,4 \mathrm{M}$ mannitol $; 15 \mathrm{mM} \mathrm{MgCl}{ }_{2} ; 4 \mathrm{mM}$ MES (pH 5,7) \\
\hline
\end{tabular}




\section{$4 \quad$ Results}

\subsection{The $P R-1$ promoter contains at least two different integration sites for SNI1-mediated suppression}

The transcriptional activator NPR1 and the repressor SNI1 are two key regulatory proteins for $P R-1$ induction. While it is assumed that NPR1 positively influences expression via interaction with members of the TGA factor family (Rochon et al. 2006), not much is known about the recruitment of SNI1 to promoter sequences to mediate repression. Since SNI1 does not contain a DNA binding domain a direct recruitment to regulatory sequences is unlikely (Mosher et al. 2006). However, at least one cis-element within the $P R-1$ promoter which contributes to SNI1-mediated repression has to be postulated. Repression can either be realized by recruitment of SNI1 to the promoter through a sequence specific DNA binding domain, or, alternatively, by recruitment of a transcriptional activator, whose expression is negatively regulated by SNI1. The critical sequence information for SNI1-dependent regulation is encoded in a $1294 \mathrm{bp} \mathrm{PR-1}$ promoter fragment as revealed by its expression pattern after INA induction in distinct mutants, which corresponds to endogenous $P R-1$ regulation (figure 4.1A). (1) The reporter gene shows an increased basal expression in snil-1 accompanied by a hyperinduction when INA is applied. (2) The 1294 promoter shows no sensitivity to INA in $n p r l-1$ and (3) this lack of induction can be partially rescued in the snil nprl double mutant. (4) The snil nprl mutant displays an increased background expression level similar to snil-1 but the hyperinduction fails likely due to the absence of the transcriptional co-activator NPR1. In conclusion, these experiments show that the SNI1/NPR1 independent pathway of $P R-1$ induction is operational on this promoter fragment. 

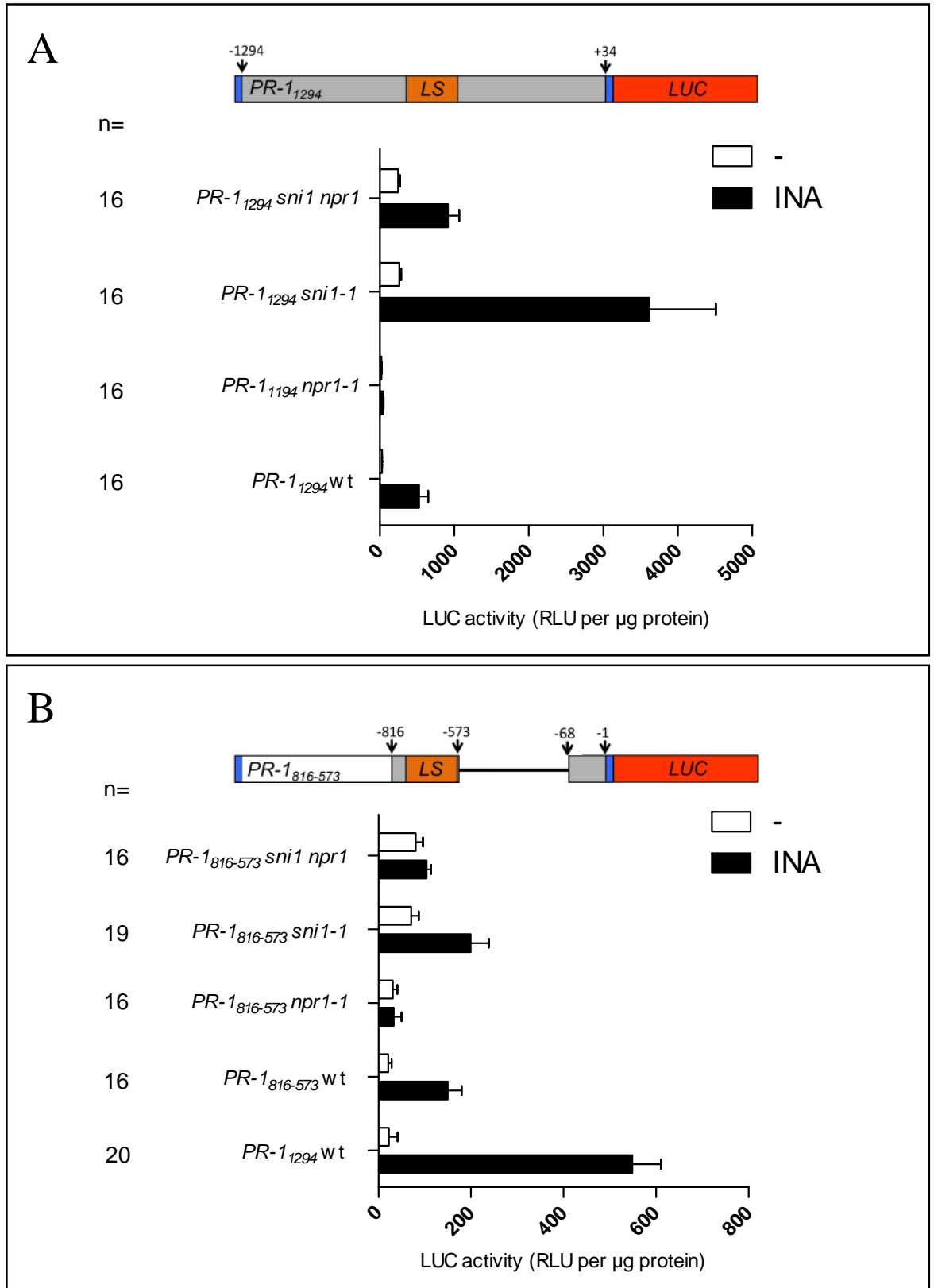

Figure 4.1: Average LUC activities of the $P R-1_{1294}(\mathrm{~A})$ and the $P R-1_{816-573}(\mathrm{~B})$ promoter fragment in wt and mutant plants.

Luciferase activities of 18-day-old plants grown on MS-plates supplemented without (white columns) or with (black columns) $30 \mu \mathrm{M}$ INA were measured. The numbers of single transgenic lines investigated for each genotype is displayed on the left $(\mathrm{n}=)$. Error bars represent the standard deviation of at least two independent experiments. The promoter derivative illustrations show $P R-1$ promoter sequences fused to a $L U C$ reporter gene. The blue boxes display the gateway ${ }^{\circledR}$ cloning sites, grey shaded regions illustrate $P R$ $1 \mathrm{cis}$-regulatory region and the white box shows sequence substitution against coding sequence derived from the $C A T$ gene. The orange $L S$-labeled region in the center of the two constructs displays the location of the $L S$-region. The gap in $P R-1_{816-573}$ illustrates sequence deletion and the arrows show relative base pair positions.

Previous promoter deletion and linker scan analysis has shown that the region between 706 to -579 (called $L S$-region for linker scan from here on) encodes different cis- 
elements that positively or negatively influence $P R-1$ promoter activity (Lebel et al. 1998). Therefore, the $L S$-region has been hypothesized to be the target for NPR1 and SNI1 activity (Kesarwani et al. 2007). One important cis-element within this region is the as-1-like element which consists of two TGACG motifs designated as LS5 and LS7. Substitution of LS5 by a linker sequence leads to slightly activated promoter activities both in the uninduced- and induced state, whereas linker scan mutagenesis of LS7 completely abolishes promoter activity, indicating that the two TGA motifs have distinct function. Because of the positive impact of $L S 7$ on $P R-1$ induction it was hypothesized that it recruits NPR1 via interaction with TGA transcription factors.

In order to gain direct evidence for the critical function of this promoter sequence with regard to NPR1- and SNI1-dependent regulation the region between -816 and -573 was fused to the basal $P R-1$ promoter (-68bp to $-1 \mathrm{bp}$ ). The $P R-1_{816-573}$ construct was transformed in the different plant genotypes (wt, nprl-1, snil-1 and nprl snil) to validate the promoter activity in comparison to the $P R-1_{1294}$ promoter (figure 4.1 B). In the wildtype background, the modified promoter construct was still inducible by INA indicating that central regulatory elements within this region are not only necessary but also sufficient for induction. The induced expression levels of $P R-1_{816-573}$ only reach approximately one third of the intensity measured in $P R-1_{1294}$ control plants suggesting additional positive $c i s$-elements outside the $L S$-region. Interestingly, the $P R-1_{816-573}$ construct shows slightly increased basal expression levels when transformed in snil-1 while the hyperinduction displayed by the $P R-1_{1294}$ construct is missing. These results suggest that SNI1 affects $P R-1$ expression in two ways. On the one hand, SNI1 suppresses basal levels of the $P R-1$ promoter via a regulatory sequence present in the $P R-1_{816-573}$ derivate. On the other hand, in contrast to the INA-induced transcription of $P R-1_{1294}$, INA induced transcription of $P R-1_{816-573}$ is not influenced by SNI1. This illustrates that $P R-1_{816-573}$ misses $c i s$-elements that can serve as target sites for positive regulators that are up-regulated in the snil-1 mutant. Though the influence of SNI1 on this modified promoter is altered, the induction of the construct is still strictly dependent on NPR1, illustrated by a complete lack of INA sensitivity in nprl-1. This confirms the notion that NPR1 may acts on the promoter through one or both of the TGACG motifs within the $L S$-region. The induction of $P R-1_{816-573}$ in the snil nprldouble mutant is abolished, likely due to the absence of sequence information that confers induction when no functional NPR1 protein is present. In conclusion, the analysis of the $P R-1_{1294^{-}}$ and the deleted $P R-1_{816-573}$ promoter in the different mutant backgrounds revealed that other positive acting regulatory sequences exist outside the $L S$-region. Moreover these 
cis-regulatory sequences contribute to hyperinduction in snil-1 illustrating that the corresponding transcriptional activators are exclusively active in snil-1 mutant background.

\subsection{W-boxes downstream of the $L S$-region contribute to $P R-1$ induction and SNI1-dependent regulation}

Further characterization of regulatory sequences outside of the $L S$-region was performed by deletion analysis of a W-box cluster located between -544 and -485. Several WRKY proteins are identified to be primary target genes of NPR1 (Wang et al. 2006) and play a role in PAMP-triggered- and SA-mediated defense responses (Dong et al. 2003; Lippok et al. 2007). Consequently it is reasonable that the $\mathrm{W}$-boxes within the $P R-1$ promoter contribute to $P R-1$ regulation although it has already been reported that this region of the promoter is not sufficient for induction (Lebel et al. 1998). Nevertheless, transcriptional regulation is often associated with synergistic actions of transcription factors and the function of these boxes was examined in the $P R-1_{1294}$ promoter context.

Deletion of W-box sequences leads to a considerable reduction of reporter gene activity (figure 4.2 A). The removal of the WRKY box designated as W1 leads to an 8-fold decrease on inducible expression compared to the $P R-1_{1294}$ control. Deletion of both or even all three $\mathrm{W}$-boxes does not yield an enhanced reduction in reporter activity, indicating that $W 2$ and $W 3$ cannot substitute for $W 1$. The impact of $W 2$ seems less severe as displayed by a moderate 3-fold reduction of induced expression levels detectable in the $\Delta W 2$ lines. This can be taken as evidence that $W 1$ may functions only if W2 is present. In summary, the most upstream located W-box (W1) seems to act as a recruitment site for a transcriptional activator to sustain the expression of $P R-1$ after induction.

Since the expression of several WRKY proteins is dependent on NPR1 and SNI1 (Mosher et al. 2006; Wang et al. 2006), it can be assumed that the lacking hyperinduction of the $P R-1_{816-573}$ reporter construct in snil-1 might be due to the absence of the $\mathrm{W}$-boxes. The promoter derivative $P R-1_{816-573}$ was therefore modified by attaching the $\mathrm{W}$-boxes $W 1$ and $W 2$. Figure 4.2 B shows that $W 1$ and $W 2$ do not alter the general expression levels under inducing- and non-inducing conditions in comparison to $P R-1_{816-573}$ in wildtype plants illustrating that further enhancer sequences are needed to reach the expression capacity of the $P R-1_{1294}$ promoter. Interestingly, the addition of $W 1$ 
and $W 2$ to $P R-1_{816-573}$ shows the hyperinducibility seen with $P R-1_{1294}$ in snil-1. This result implies that the $\mathrm{W}$-boxes $W 1$ and $W 2$ integrate SNI1-dependent signals by recruitment of different amounts or combinations of SNI1-regulated WRKY transcription factors. These WRKY factors are thus candidates which mediate the increased transcriptional activation in snil-1 and are necessary for the induction of the PR-1 promoter in snil nprl.

\subsection{The as-1-like element is dispensable for INA induced activation of PR-1}

The LS-region between -704 and -577 contains an as-1-like element, which is assumed to be the putative integration site for NPR1-dependent $P R-1$ regulation. The TGACG motifs of this element are also referred to as $L S 5$ and $L S 7$, since they were identified by linker scan mutagenesis. Surprisingly, substitution of both motifs generates a promoter derivative, that reveals a similar induction like the $P R-1_{1294}$ promoter though basal- and induced expression levels are increased (Figure 4.3). This result illustrates that the as-1like element is dispensable for $P R-1$ induction and that an as-1-independent mechanism can complement the loss of TGA factor-controlled regulation. This as-1-independent regulation depends on the $\mathrm{W}$-box containing element $L S 4$ as shown by substantially decreased INA-induced reporter gene activities in $L S 4 / 5 / 7_{\text {mut }}$ plants. In conclusion, two alternate pathways are merging on the $L S$-region: The as-1-regulated pathway, which is slightly negatively influenced by $L S 4$ (see $L S 4_{m u t}$; Figure 4.3), and the $a s$-1-independent pathway, which is strongly dependent on LS4.

In the absence of $L S 5$, the promoter is inducible to the same extent as $P R-1_{1294}$ and $L S 5 / 7_{\text {mut }}$. The activation is due to the as-1-independent pathway, as shown by the lack of activation in $L S 4 / 5_{\text {mut }}$, which lacks the W-box that is essential for the as-1independent regulation. Thus $L S 5$ represses the alternate pathway. The inactivity of the $L S 4 / 5_{\text {mut }}$ construct also shows that the as-1-dependent regulation can only function if $L S 5$ and $L S 7$ are present. 


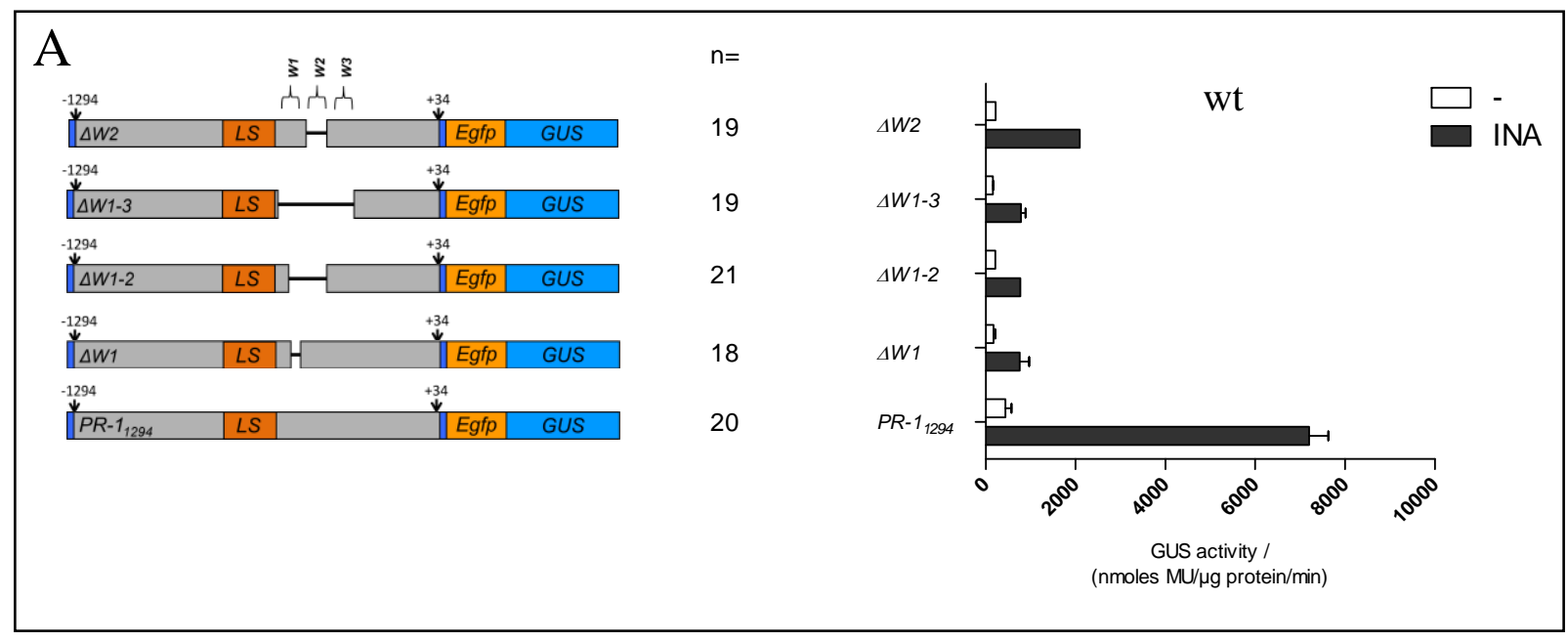

B

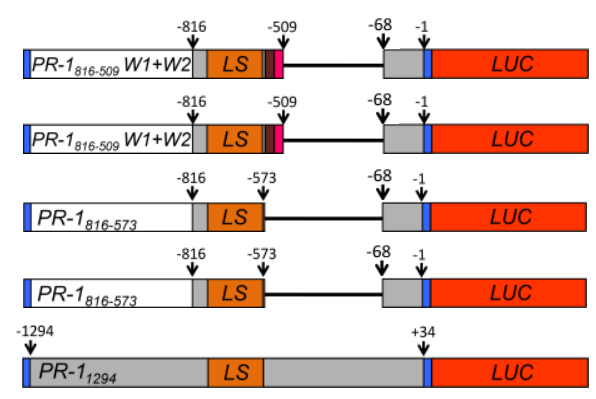

$\mathrm{n}=$

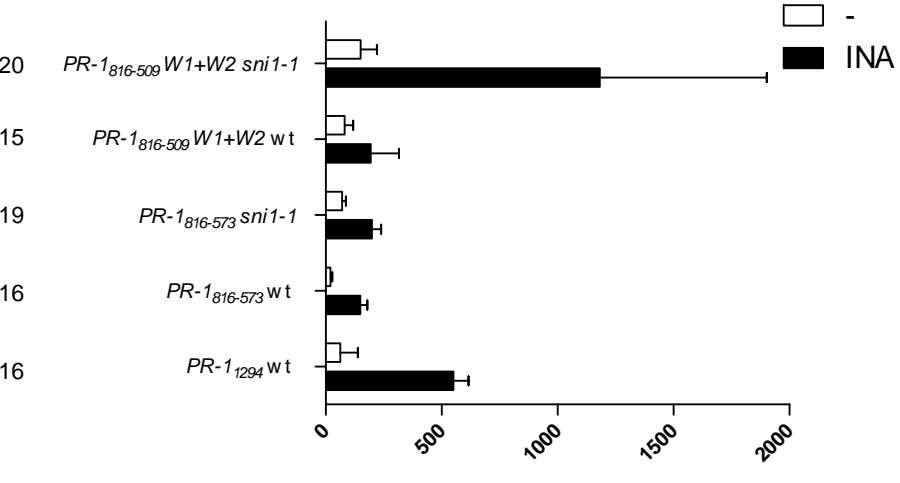

LUC activity (RLU per $\mu$ g protein)

Figure 4.2: Average activities of $P R-I_{1294}$ promoter constructs lacking certain $W$-boxes in wt (A) and of $P R-1_{816-573}$ promoter constructs with and without an attached regulatory $\mathrm{W}$-box region in wt and snil-1 (B).

Figure A shows relative GUS activities of reporter gene lines grown for 18 days on MS-plates supplemented with- or without $30 \mu \mathrm{M}$ INA. The lines are carrying distinct mutations in the W-box cluster. In $\mathbf{B}$, the relative LUC activities of the $P R-1_{816-509} W 1+W 2$ reporter plants are displayed in comparison to the $P R-1_{816-573}$ promoter derivative. The numbers of individual lines tested in each experiment are indicated $(\mathrm{n}=)$, the error bars represent the standard deviation of two independent experiments. The promoter derivative illustrations show $P R-1$ promoter sequences fused to an $E g f p / G U S$ (A) or a $L U C(\mathbf{B})$ reporter gene. The blue boxes display the gateway ${ }^{\circledR}$ cloning sites, grey shaded regions illustrate $P R-1$ cisregulatory regions and the white box shows sequence substitutions against the coding sequence of the $C A T$ gene. The orange $L S$-labeled box in the center of the constructs displays the location of the $L S$ region. The two attached W-boxes to the modified promoter construct are labeled red and pink. The arrows and the numbers show relative base pair positions, gaps illustrate deleted sequences. 
In the absence of $L S 7$, the promoter is only weakly inducible, showing that both pathways do not work efficiently. The residual induction is dependent on the as-1independent pathway as revealed by the further reduction when mutating LS4. In conclusion, the as-1-regulated pathway is compromised upon mutation of either LS5 or $L S 7$ or both, and the as-1-independent pathway is efficiently repressed by LS5 and less efficiently by $L S 7$ and functions best when both elements are missing.

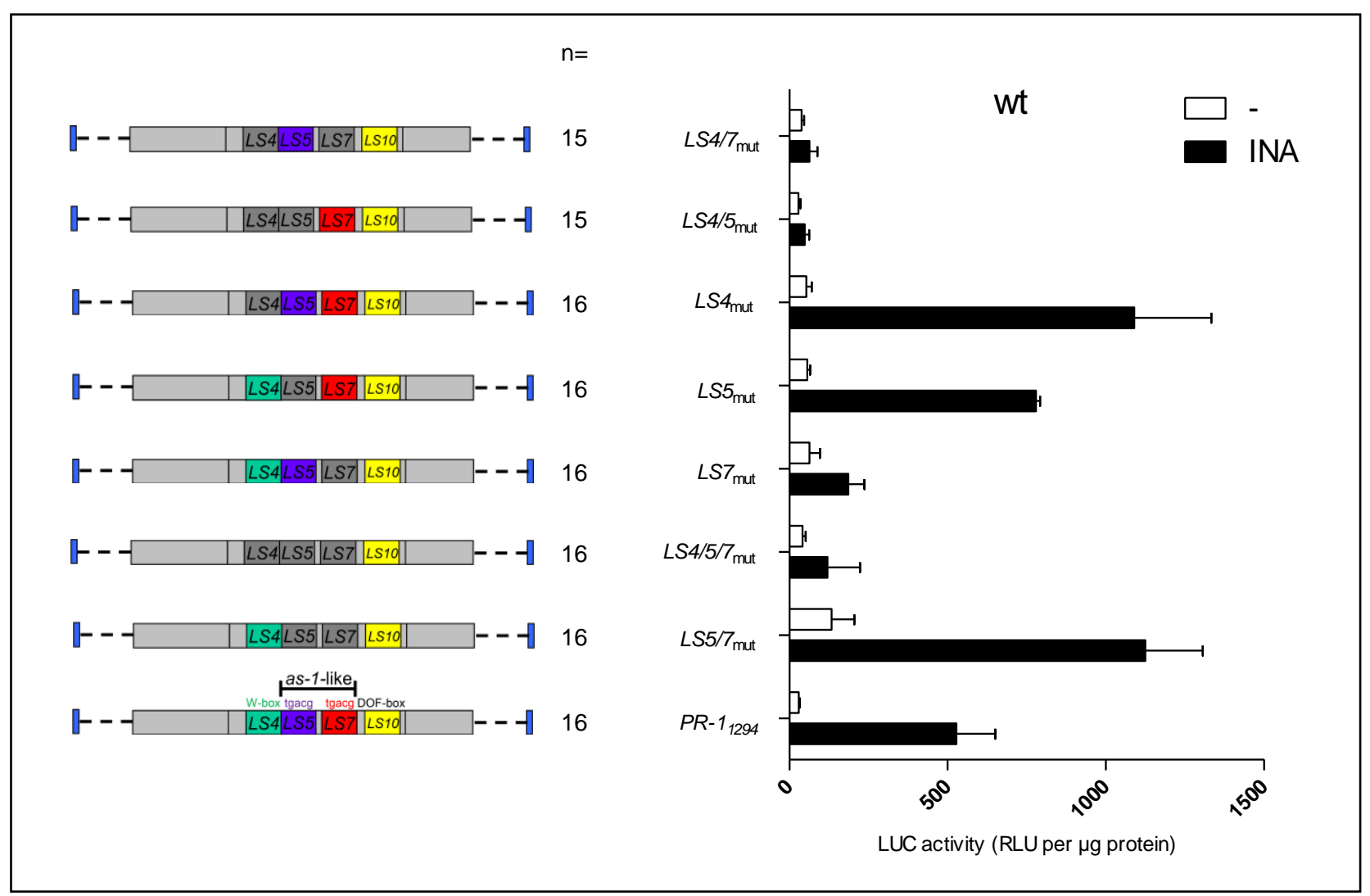

Figure 4.3: Average LUC activities of promoter derivatives carrying distinct mutations in the as-1like element and the adjacent W-box $L S 4$ in wildtype background.

The figure shows the basal- and induced expression levels of 18-day-old transgenic reporter gene lines grown axenically on MS-plates supplemented with- or without $30 \mu \mathrm{M}$ INA. The number of individual lines tested for each construct is indicated $(\mathrm{n}=)$ and the error bars illustrate the standard deviation of two independent experiments. The promoter figures on the left show a magnified view on the $L S$-region. The grey shaded boxes indicate 10bp sequence substitutions in the corresponding cis-elements (for details see supplemental data).

The influence of the repressor SNI1 on the as-1-independent regulatory pathway was further examined by analysis of the promoter constructs $L S 4_{\text {mut, }} L S 5_{\text {mut }}, L S 7_{\text {mut }}$ and $L S 5 / 7_{\text {mut }}$ in snil-1 (figure 4.4). While mutation of $L S 4$ leads to a 2 -fold increased 
expression in relation to the $P R-1_{1294}$ promoter in snil-1, the constructs harboring mutations in one or both TGACG motifs displayed decreased induced expression levels, which were similar to the values obtained in the wildtype (ca. $1000 \mathrm{U}$, see Figure 4.3). This illustrates that the as-1-independent pathway is not influenced by SNI1 in the induced state and that the as- 1 element is required for the hyperinduction exhibited by the $P R-1_{1294}$ promoter in snil-1. It seems plausible that NPR1, which is recruited to the TGA factors bound to the as-1-like element, is responsible for this hyperinduction. This notion is consistent with the decreased induced expression of the endogenous promoter in snil nprl when compared to the hyperinduction detected in snil-1 (see figure 4.1). Furthermore, mutation of TGA recruitment sites considerably increased the basal expression of the reporter lines revealing a negative impact of TGA factors on $P R-1$ transcription when SNI1 is absent, a phenomenon previously observed when analyzing endogenous $P R-1$ expression in a tga2 snil double mutant (Kesarwani et al. 2007). As background levels rise and induction is not altered the constructs only show weak ( $L S 5_{\mathrm{mut}}$ and $\left.L S 5 / 7_{\mathrm{mut}}\right)$ or no ( $\left.L S 7_{\mathrm{mut}}\right)$ induction at all.

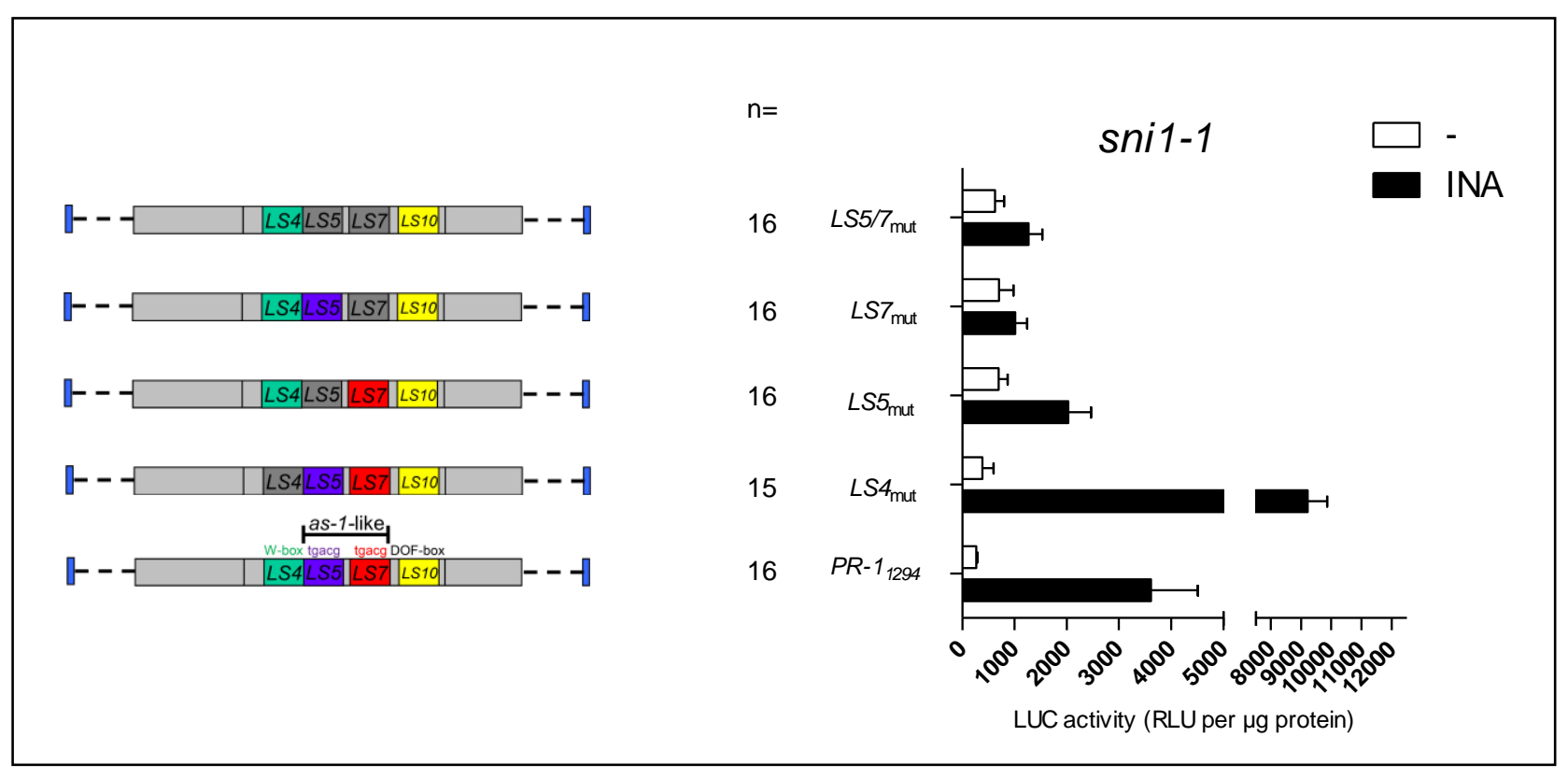

Figure 4.4: Average LUC activities of promoter constructs carrying sequence alterations in the TGACG motifs or the W-box element LS4 in snil-1.

The figure shows the expression of 18-day-old transgenic reporter gene lines axenically grown under inducing- and non-inducing conditions. The number of transgenic lines examined is indicated $(\mathrm{n}=)$, the error bars indicate the standard deviation of at least two independent experiments. The promoter illustrations on the left show the elements $L S 4, L S 5, L S 7$ and $L S 10$ located within the $L S$-region. The grey shaded boxes indicate 10bp sequence substitutions in the corresponding cis-elements (for details see supplemental data). 
The inducibility of the $L S 5 / 7_{\text {mut }}$ construct indicates that the $P R-1$ promoter can be activated in the absence of the as-1-like element, which is the recruitment site for the NPR1/TGA complex. Next, we asked the question, whether the as-1-independent regulatory pathway is also independent from NPR1 and transformed the $L S 5 / 7_{\text {mut }}$ construct into the nprl-1 mutant. Analysis of the resulting transgenic lines indicated that the promoter was severely compromised, indicating that NPR1 is needed for this pathway. It seems likely that transcription of WRKY transcription factors recruited to either $L S 4$ or the W-box cluster downstream of the $L S$-region depends on NPR1.

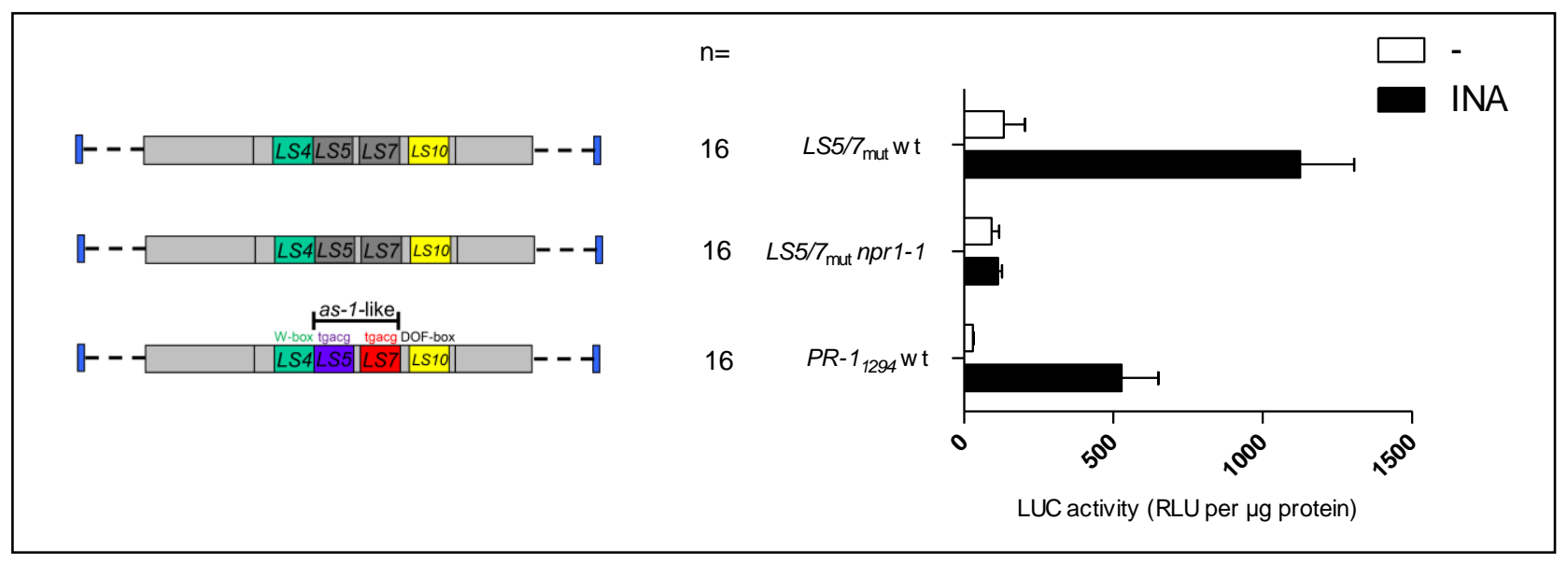

Figure 4.5: Average LUC intensities of $L S 5 / 7_{\text {mut }}$ reporter gene lines in wt and $n p r 1-1$ mutant background.

18-day-old plants axenically grown on 30 $\mu \mathrm{M}$ INA- or control MS-plates were used for protein extraction and subsequent LUC assays. The error bars show the standard deviation of two experiments, the number of transgenic lines examined for each promoter construct is indicated $(n=)$. The dark grey shaded boxes in the promoter illustrations on the left indicate sequence substitutions in the TGACG motif-containing elements LS5 and LS7.

Further experiments in the snil-1mutant have been performed with the loss-of-induction constructs $L S 4 / 5_{\text {mut }}$ and $L S 4 / 7_{\text {mut }}$ to evaluate the influence of SNI1 on these promoter derivatives.

Interestingly, $L S 4 / 5_{\text {mut }}$ and $L S 4 / 7_{\text {mut }}$ act different in snil-1 as inducibility of $L S 4 / 5_{\text {mut }}$ is partially re-established while $L S 4 / 7_{\text {mut }}$ even shows decreased expression when treated with INA (figure 4.6). This implies that a non-mutated LS7 element is essential for the induction of the $L S 4 / 5_{\text {mut }}$ promoter construct in snil-1, possibly due to a synergistic 
activation mediated by a TGA/NPR1 complex at $L S 7$ and deregulated WRKY transcription factors at the W-box cluster. In addition, $L S 7$ maybe recruits SNI1 in case of a mutated $L S 5$ site and thereby inhibits induction of the $L S 4 / 5_{\text {mut }}$ derivative in wildtype plants. Further evidence for the implication of the $\mathrm{W}$-box cluster in the activation of $L S 4 / 5_{\text {mut }}$ was gained by analysis of the $L S 4 / 5_{\text {mut }} \Delta W 1-3$ construct in snil-1 mutant background (figure 4.7). In contrast to $L S 4 / 5_{\text {mut }}$ this promoter derivative totally lacks sensitivity to INA in snil-1. Figure 4.7 also displays once more that the W-box W1 is involved in snil-1 entailed hyperinduction as the induced expression levels of $\Delta W 1$ lines are considerably lower than the levels monitored with the $P R-1_{1294}$ control promoter.

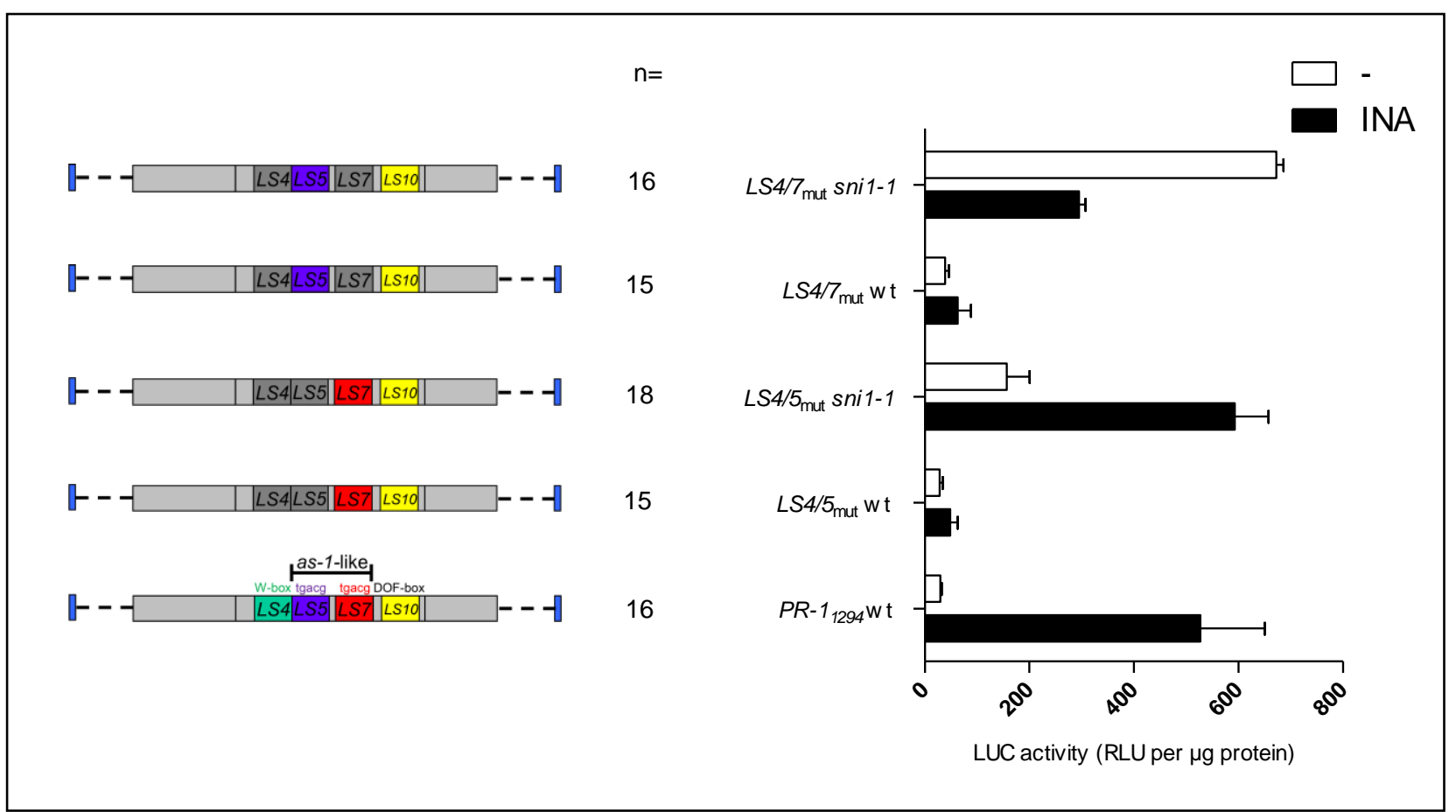

Figure 4.6: Average LUC activities of lines carrying differing sequence mutations in the elements $L S 4, L S 5$ and $L S 7$ in wildtype and snil-1 mutant background.

The LUC activities depicted in the figure were obtained from 18-day-old plants axenically grown on MSmedium supplemented with- or without $30 \mu \mathrm{M}$ INA. The numbers $(n=)$ indicate measured transgenic lines and the error bars represent two independent experiments. The dark grey shaded boxes in the promoter illustrations on the left indicate sequence substitutions in the TGACG motif-containing elements $L S 5$ and $L S 7$ and the putative W-box $L S 4$. 


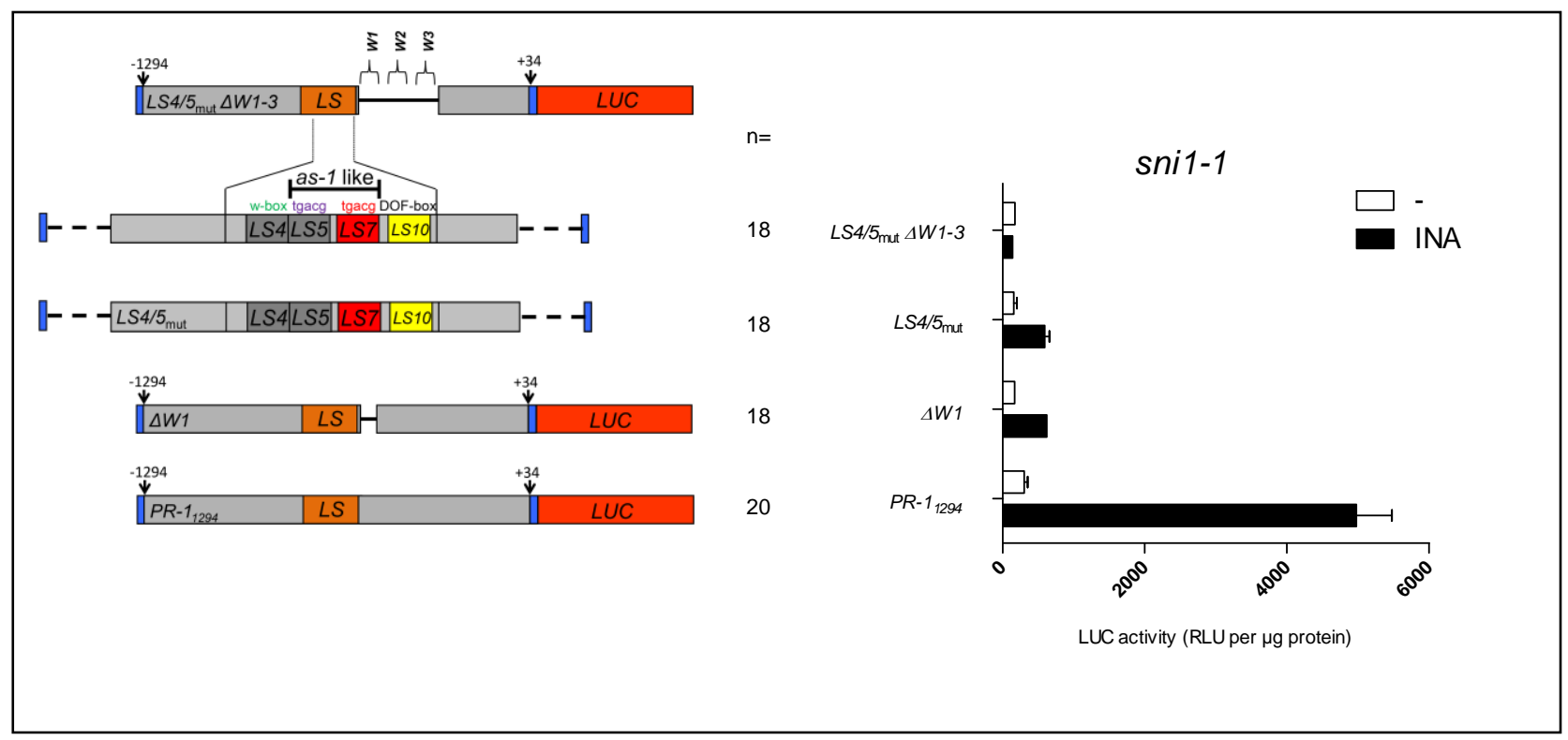

Figure 4.7: Average relative LUC activities of constructs harboring distinct mutations or deletions in the elements $L S 4, L S 5, \triangle W 1$ and $\triangle W 1-3$.

The 18-day-old plants were grown on MS-plates supplemented with- or without 30 $\mu \mathrm{M}$ INA. The error bars illustrate the standard deviation of two experiments, the number of investigated transgenic lines is indicated $(n=)$. Dark grey shaded boxes in the promoter illustrations on the left indicate sequence substitutions in the elements $L S 4$ and LS5, gaps show sequence deletions of $W 1$ and $W 1-3$ respectively. Blue boxes display the gateway® cloning sites, grey shaded regions illustrate $P R$-lpromoter sequence. The orange $L S$-labeled box in the center of the constructs displays the location of the $L S$-region.

\subsection{Alteration of the distance between the palindromic repeats of the as-1-like element interferes with INA induced expression}

The experiments presented so far show that the as-1-like element within the $P R-1$ promoter contains putative target sites for TGA factor-mediated NPR1 recruitment (LS7 and LS5) and a target site (LS5) for a repressor, which suppresses the as-1-independent activation pathway. Data published previously indicate that the distance between the two palindromic repeats of the as-1 element is critical for the NPR1 dependency of SAinducible promoters (Blanco et al. 2005; Krawczyk et al. 2002). The classical distance between the TGA recruitment sites is $4 \mathrm{bp}$ as displayed by the $35 S$, GST6, and CYP81D11 promoters whereas the distance in the NPR1-dependent $P R-1$ promoter is $9 \mathrm{bp}$.

Therefore, it was tested whether a changed spacing between LS5 and LS7 somehow influences the expression of the $P R-1$ promoter. For this purpose, the sequence ctattttac 
was substituted against the sequence $a a a a$ and the corresponding construct was termed LS5aaaaLS7 (figure 4.8). The promoter derivative shows a substantial reduction in inducible gene expression illustrating an impaired NPR1 regulation at the mutated as-1like element. In snil-1, the INA-induced activity is as high as in the construct lacking the $a s-1$-like element, indicating that the $a s-1$-independent pathway is operational. This can be taken as evidence that SNI1 represses the as-1-independent pathway through LS5. As LS5aaaaLS7 cannot be efficiently activated by NPR1, the promoter is inactive. In the snil-1 mutant background, repression of the as-1-independent pathway does not occur, allowing high expression levels after INA treatment. The basal expression of LS5aaaaLS7 in snil-1 is slightly enhanced when compared to the expression levels of $P R-1_{1294}$ and slightly decreased when compared to the expression levels of $L S 5 / 7_{m u t}$. Thus, TGA factors are still recruited to the LS5aaaaLS7 as-1-like element and partially suppress the basal promoter activity in snil-1. Consistently, the induced- and noninduced expression levels of LS5aaaaLS7 in snil-1 look similar to the expression levels obtained with the $P R-1_{1294}$ construct in snil nprl (figure 4.1).

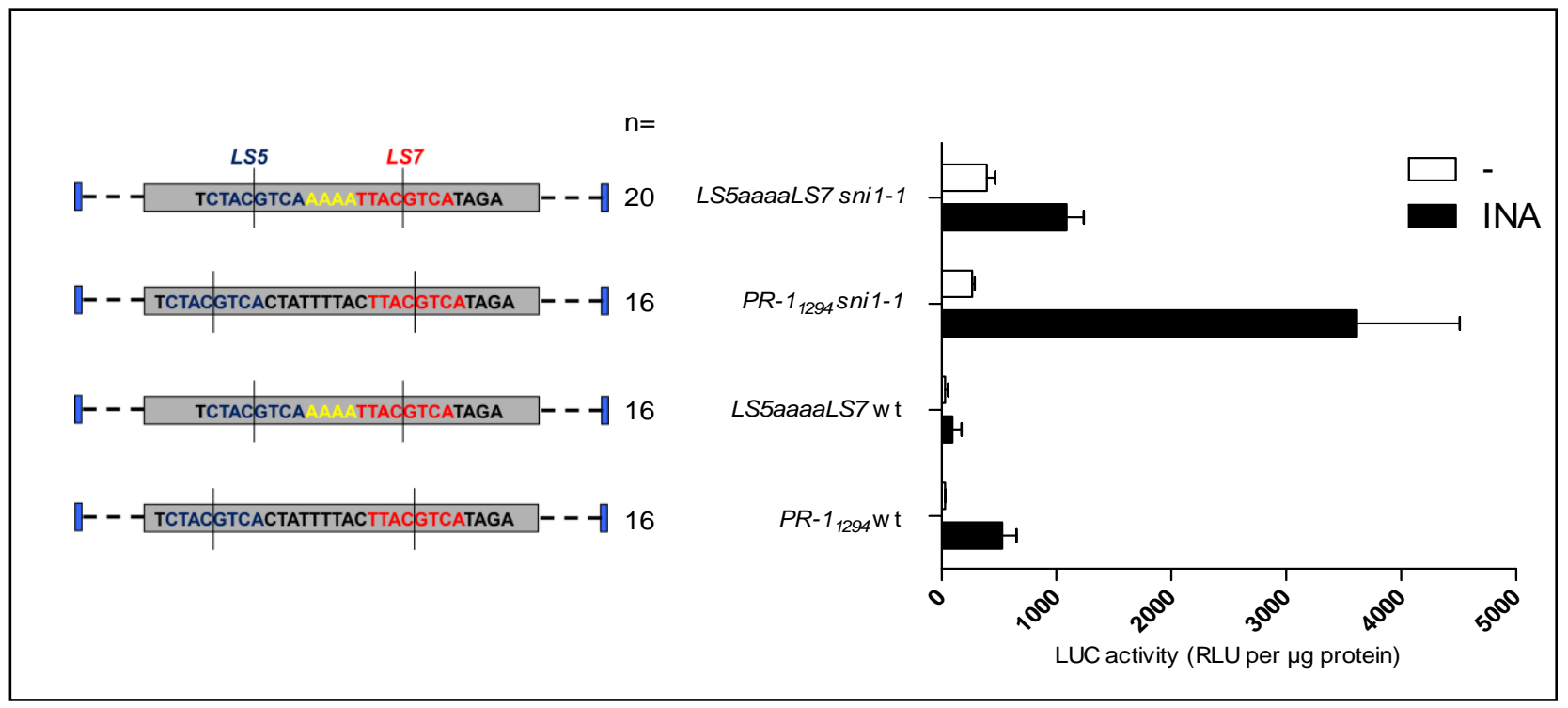

Figure 4.8: Relative LUC activities of a promoter derivative which harbors a mutated linker sequence between $L S 5$ and $L S 7$ (LS5aaaaLS7) and thereby simulates the distance between the palindromic repeats known from NPR1-independent promoters.

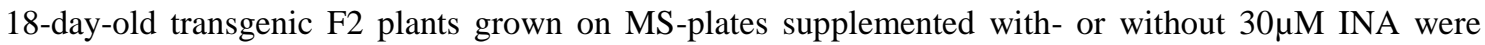
used for the experiment. The number of investigated lines per construct is indicated $(\mathrm{n}=)$, error bars represent the standard deviation of two independent induction treatments. The inserted aaaa sequence is labeled yellow in the promoter illustrations on the left. LS5 (blue) and LS7 (red) mark the two TGACG motifs. 


\subsection{The CaMV 35S as-1 element confers deregulated high expression in the $P R-1$ promoter context}

So far, our analysis suggests that LS5 and SNI1 repress the as-1-independent SAinduced pathway and that recruitment of NPR1 to the as-1-like element is therefore required for activation. However, this recruitment seems to be impaired when the distance between $L S 5$ and $L S 7$ is reduced by 5 bps. Next we asked the question, if the as-1-element of the CaMV $35 \mathrm{~S}$ promoter, which contains two TGACG motifs in a distance that does not allow NPR1 action, would also be able to recruit SNI1. Therefore, the as-1-like element of the $P R-1$ promoter was replaced by the $35 S$ as-1 element. Figure 4.9 displays the relative LUC activities of the substituted as-1 element lines transformed in wt, snil-1 and nprl-1. The replacement of this regulatory sequence causes a substantial 400-fold increase of basal- and 50-fold increase of induced gene expression in relation to the $P R-1_{1294}$ wt control lines. This indicates that the $35 S$ as -1 element does not confer repession as observed for the LS5aaaaLS7 element, but rather recruits a strong activator. As the $35 S$ as- 1 element itself confers only weak SA- and auxin-induced gene expression, strong synergistic interactions with cis-elements of the $P R-1$ promoter seem to occur. These interactions are less strong in the nprl-1 mutant, indicating that some of these trans-factors are expressed in an NPR1-dependent manner. INA treatment leads to an approximately twofold induction of the reporter gene activity independent of the genetic background. Putative recruitment sites for additional NPR1dependent regulators are once again the $\mathrm{W}$-boxes downstream of the $L S$-region. This hypothesis was tested by integrating the $35 S$ as- 1 element in the W-box cluster-lacking $P R-1_{816-573}$ promoter derivative. 


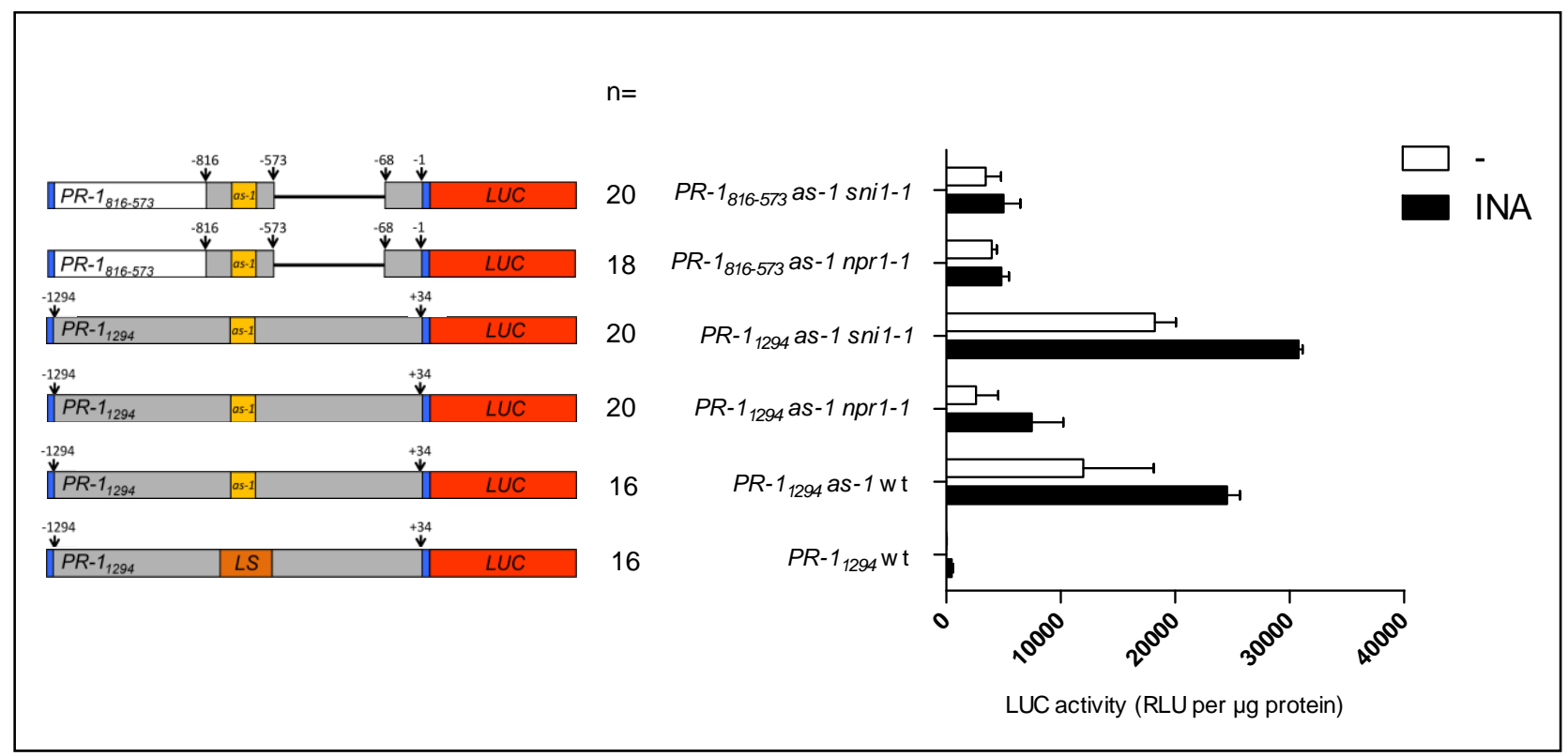

Figure 4.9: Average LUC activities of $P R-1_{1294}$ as-1- and $P R-1_{816-573}$ as-1 promoter constructs carrying substituted $a s-1$ elements derived from $\mathrm{CaMV} 35 \mathrm{~S}$.

The figure shows the LUC activities of 18-day-old plants axenically grown on MS-plates supplemented with- or without $30 \mu \mathrm{M}$ INA. The influence of the genetic background on $P R-1_{1294}$ as- 1 - and $P R-1_{816-573}$ as-1 promoter activity is displayed, error bars indicate the standard deviation of two experiments while the numbers $(n=)$ illustrate the amount of transgenic lines tested. The gaps in the promoter illustrations on the left denote deletions, the white boxes within the $P R-1_{816-573}$ as- 1 constructs display sequence substitutions. The substitution of the as-1-like element against the $35 \mathrm{~S}$ as-1 element is indicated by the yellow box in the center of the constructs, the $L S$-region in the $P R-1_{1294}$ promoter is colored orange. The blue boxes display the gateway ${ }^{\circledR}$ cloning sites, grey shaded regions illustrate $P R-1$ cis-regulatory regions and the white boxes show sequence substitutions against the coding sequence of the $C A T$ gene. The arrows and numbers show base pair positions.

As illustrated in figure 4.9, the different reporter gene activities in $n p r-1-1$ and snil-1 are gone when the $P R-1_{816-573}$ as -1 construct is integrated in these mutants. The loss of NPR1 dependency is accompanied with a lower reporter gene activity in comparison to the $P R-1_{1294}$ as -1 promoter and a general loss of inducibility. The experiments presented here show that recruitment sites for NPR1-dependent transcription factors are missing in the $P R-1_{816-573}$ as-1construct. Candidate sites are the W-boxes downstream of the $L S$ region since it is reported that the induction of many WRKY proteins is dependent on NPR1 (Dong et al. 2003; Wang et al. 2006). 


\subsection{The sequence between the TGA binding sites of the as-1 element is critical for the increased constitutive expression observed in the as-1 reporter plants}

The strong expession levels conferred by the $35 S$ as- 1 element but not by the PR-1derived as-1-like element evoke the question, which sequence within this regulatory unit is responsible for this difference. Common features are, that both elements contain two variants of the TGACGTAC palindromes spaced by a "linker" sequence. We generated a number of chimeric as-1 elements containing TGA-recruiting sequences and sequences between these motifs either derived from the $P R-1$ - or the $35 S$ as- 1 element.

The respective constructs were fused to the GUS reporter gene and analyzed in transgenic Arabidopsis plants. Surprisingly, the $35 \mathrm{~S}$ as-1 element conferred increased basal levels, which reached $50 \%$ of the induced levels of $P R-1_{1294}$, but were not 400 fold higher as observed with the LUC reporter gene (Figure 4.10 A). Induced levels were similar to $P R-1_{1294}$, which is in contrast to the 50 -fold higher expression levels observed with the $L U C$ construct. An explanation for this discrepancy could be that strong silencing effects occur when the rate of transcription is severely increased in the GUS reporter plants leading to attenuated signal intensities. Though the overall expression is weaker, the constitutive activation mediated by the substituted as-1 element can still be taken as a read-out for addressing the above mentioned questions.

Constitutive activation of the promoter occurs when the $P R-1$ linker sequence is substituted against the linker derived from the $35 \mathrm{~S}$ as-1 element as displayed by the LS5gggaLS7 construct. Activation is not influenced by NPR1, supporting our previous assumption that NPR1 cannot be recruited to promoters that contain as-1-like elements that are spaced by only 4 bps. Whereas LS5aaaaLS7 construct (see 4.8) is repressed presumably by SNI1, LS5gggaLS7 is active. It is concluded that the GGGA-linker sequence of the $a s-1$ element interferes with repression of the promoter by SNI1. 

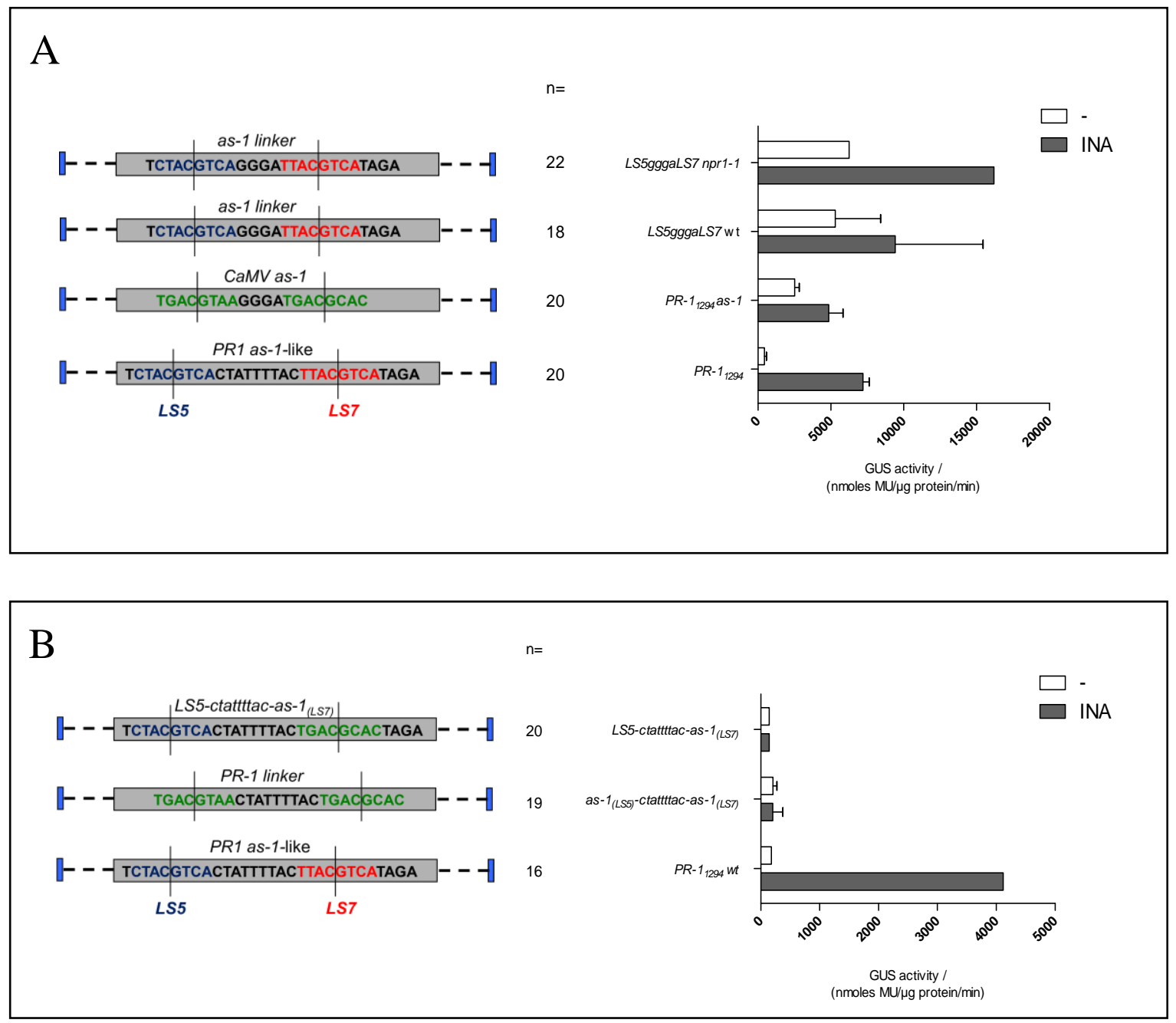

Figure 4.10: GUS activity measurements of distinct promoter derivatives containing alterations in the composition of the as-1 element.

The MUG assays were performed with plants axenically grown on $30 \mu \mathrm{M}$ INA- or control MS-plates. The error bars in figure $\mathbf{A}$ and $\mathbf{B}$ represent the standard deviation of two independent experiments, in case of lacking error bars the lines were examined just one time. The numbers of single lines tested in each experiment are indicated $(\mathrm{n}=)$. as- 1 sequence alterations are illustrated in the promoter diagrams, all mutations were integrated in the $P R-1_{1294}$ promoter context.

The integration of the 9bp PR-1 linker sequence within the substituted as- 1 element (as$1_{(L S 5)}$-ctattttac-as- $\left.1_{(L S 7)}\right)$ leads to loss of basal expression and INA sensitivity indicating that the TGACG motifs of the as-1 element cannot complement the function of the TGA recruitment sites of the $P R-1$ promoter. The failure of the promoter construct to induce gene expression is probably due to the sequence of the TGACG motif in the LS7 position of the as-1 element. Whereas $L S 7$ of the $P R-1$ promoter contains the central ACGT palindrome, the downstream TGACG motif of the $35 S$ as- 1 sequence $\left(\right.$ as $\left.-1_{(L S 7)}\right)$ misses the $\mathrm{T}$ and might therefore constitute a weak binding site, that can only be occupied when another binding site is present at a distance (4 bps) allowing cooperative 
interactions (Krawczyk et al. 2002). Consistently, INA induction also fails in the LS5ctattttac-as- $1_{(L S 7)}$ promoter derivative (figure 4.10 B).

\subsection{The first base within the as- 1 linker region determines the overexpression capacity of the as-1 promoter construct}

The experiments conducted with the transgenic reporter gene lines revealed a central role of the $35 S$ as- 1 linker sequence for the increased promoter activity. A more detailed analysis of this $4 \mathrm{bp}$ spanning sequence was performed by transient protoplast transformation experiments using $P R-1_{1294}$ as 1 constructs carrying distinct sequence alterations in the critical linker region.

In our hands, transiently transformed protoplasts are not an optimal system to investigate $P R-1$ expression due to a high background activity of transiently introduced promoter constructs and the failure of SA- and INA-mediated induction. Nevertheless, $P R-1$ promoter constructs containing the $35 S$ as- 1 element instead of the L5-LS7 region show increased expression known from the results obtained with transgenic plants (figure 4.11 A). Thus, the sequence requirements of the $35 S$ as- 1 element can be studied in this system. The $P R-1_{816-573^{-}}$and $P R-1_{816-573} a s-1$ promoter derivatives show weaker expression levels than the corresponding $P R-1_{1294}$ constructs. Thus, the constitutive activity of the promoter might be due to de-repressed WRKY-transcription factors binding to this region. Consistently, the expression of the $P R-1_{1294}$ promoter fragment in protoplasts was essentially abolished when the W-box cluster was deleted (figure 4.11 B). Interestingly, the constructs $a s-1_{(L S 5)^{-}}$ctattttac-as-1 $1_{(L S 7)}$ and LS5aaaaLS7, which are inactive in the transgenic situation, are as strongly expressed as $P R-1_{1294}$, indicating that the repressive effect of LS5/SNI1 may not be operational any more. 

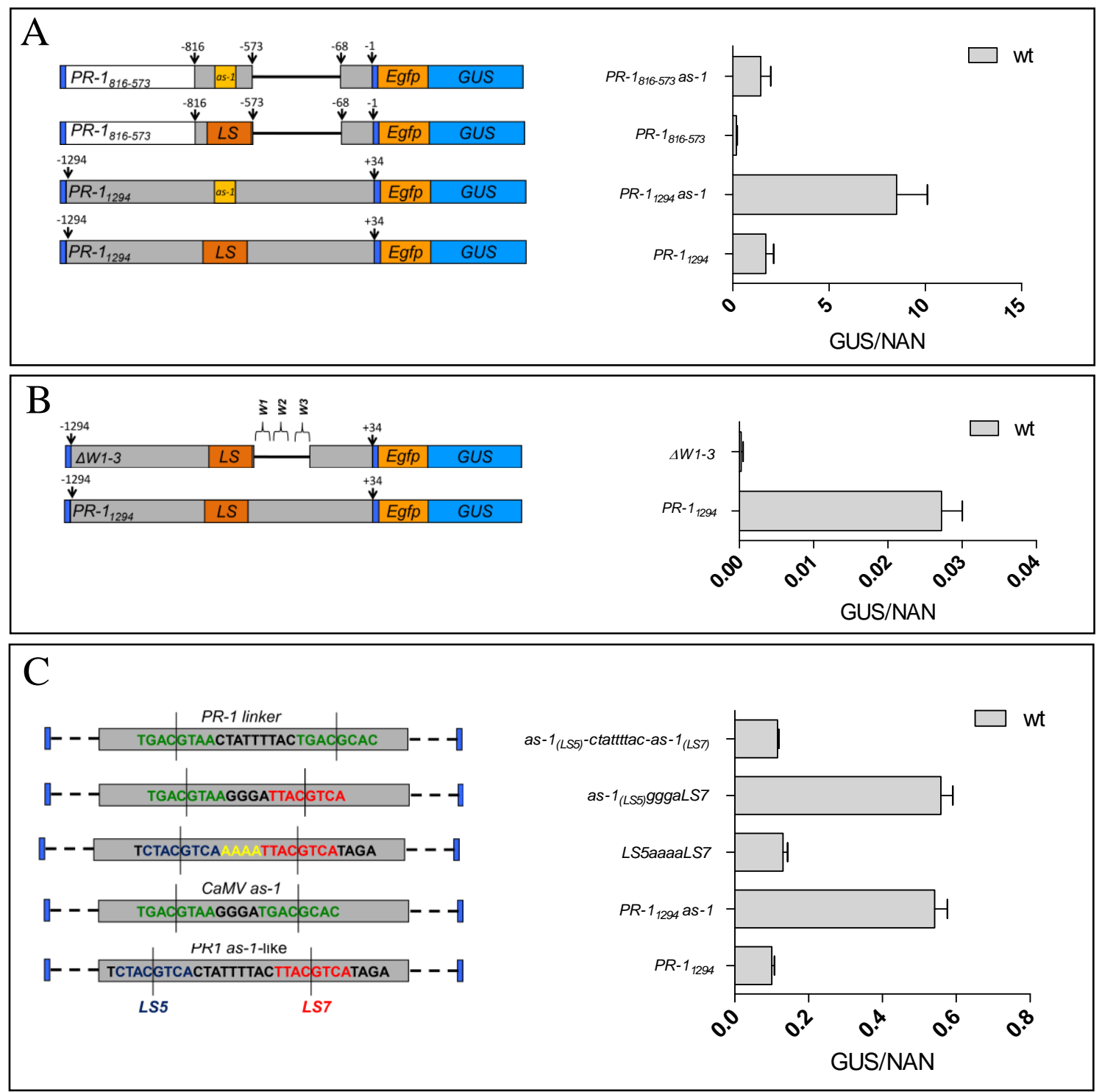

Figure 4.11: Transient expression analysis of distinct $P R-1$ promoter constructs carrying sequence alterations at the site of the as-1-like element $(\mathrm{A}+\mathrm{C})$ or a $\mathrm{W}$-box cluster deletion $(\mathrm{B})$.

The figures show GUS activities obtained from transient transformations of distinct promoter:GUS constructs in A. thaliana protoplasts. Leaves of 3-week-old soil grown and non-induced wt plants were used for protoplast isolation. The y-coordinates demonstrate the ratio of GUS activity to the internal NAN standard. Figure A shows the impact of the $35 S$ as- 1 element in the $P R-1_{1294^{-}}$or $P R-1_{816-573}$ promoter context on increased reporter gene activity, while $\mathbf{B}$ displays the effect of a $\mathrm{W}$-box cluster deletion (between -546 to -484) on constitutive expression. In $\mathbf{C}$ the increased reporter activities of constructs with integrated GGGA linker sequence are illustrated in combination with as-1-mutated constructs which do not cause enhanced expression. The error bars represent the standard deviation derived from 4 replicates. The orange $L S$-labeled boxes in the promoter diagrams in $\mathbf{A}$ and $\mathbf{B}$ illustrate the $L S$-region; the smaller yellow boxes display as-1 element substitutions against $35 S$ as-1. Blue boxes display the gateway® cloning sites, grey shaded regions illustrate $P R-1$ cis-regulatory regions and the white box shows sequence substitutions against the coding sequence of the $C A T$ gene. Arrows and numbers show base pair positions. The sequences of the as- 1 element mutations examined in $\mathbf{C}$ are indicated in the promoter illustrations. $L S 5$ is labeled blue, $L S 7$ red, and the TGACG motifs of the $35 S$ element are labeled green. The bars indicate the palindromic centers of the TGACG motifs. 


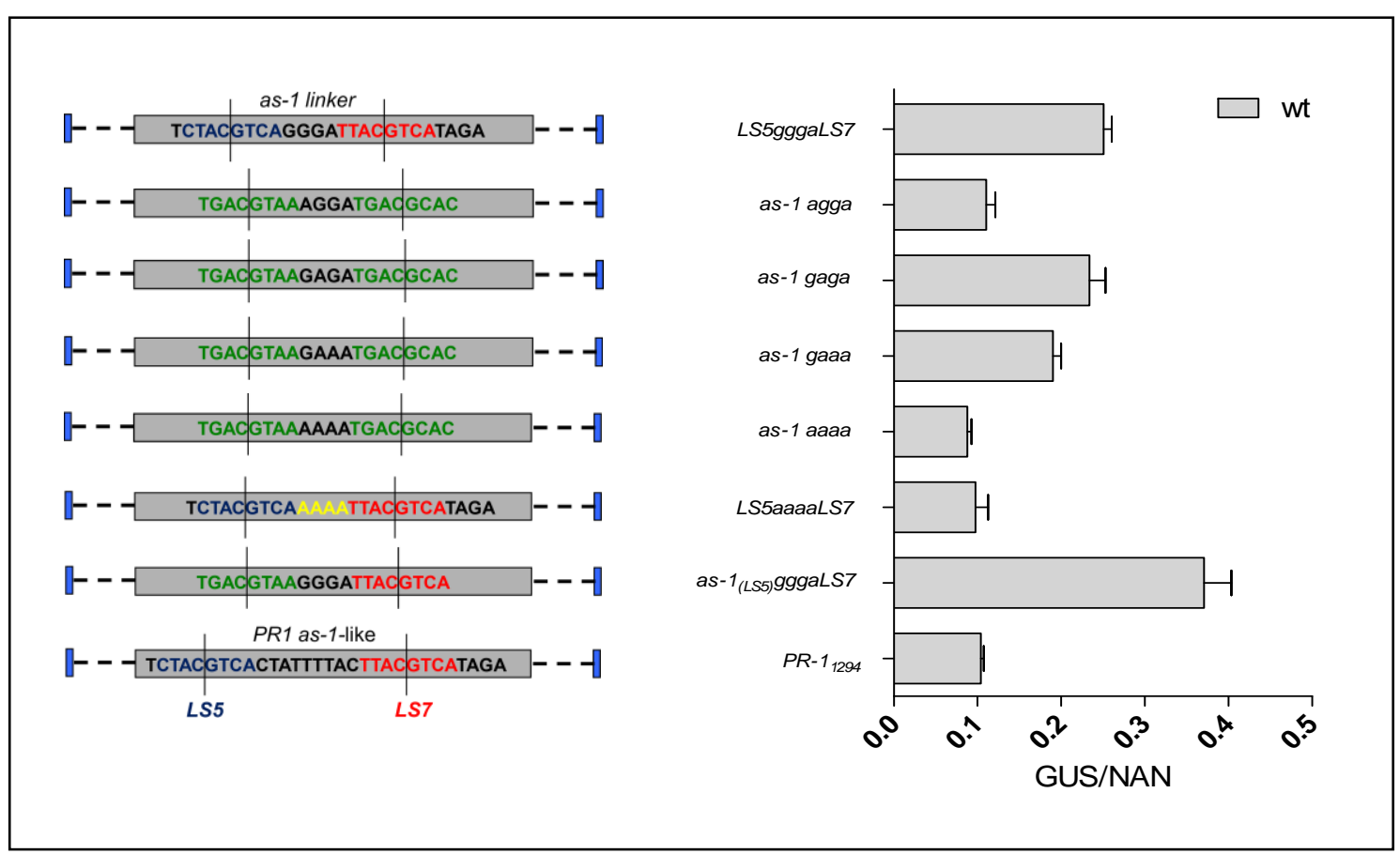

Figure 4.12: Analysis of the $35 S$ as-1 linker sequence with regard to transcriptional activation.

Leaves of 3-week-old soil grown and non-induced wt plants were used for protoplast isolation and subsequent transformation. The influence of the $a s-1$ linker sequence on increased promoter activity is displayed. Error bars represent the standard deviation of 4 replicates. The TGACG sequences derived from the $P R-1$ promoter are dyed blue (LS5) and red (LS7), while the TGA recruitment sites of the $35 S$ as- 1 element are colored green. Bars indicate the palindromic centers of the TGACG motifs, all mutations are integrated in the $P R-1_{1294}$ promoter context.

In figure 4.12 the GUS activities of $P R-1_{1294} a s-1$ constructs harboring different linker sequences are displayed. The first base within the $35 S$ linker seems to be critical for $a s$ 1 -mediated activation as revealed by the $P R-1_{1294}$ as-1 gaaa construct, which shows increased reporter gene activity as compared to $P R-1_{1294}$ as- 1 aaaa. The $P R-1_{1294}$ as -1 agga derivative indicates wildtype background expression, again supporting the relevance of the first guanine. 


\subsection{Substitution of the as-1 element within the $P R-1$ promoter changes the influence of subgroup II TGA factors on expression}

The analysis of the function of the $35 S$ as -1 element in the $P R-1$ promoter context implies that the linker sequence between the palindromes determines whether an activating protein can be recruited. This activator might interfere with the repression of the promoter through LS5. Class II TGA factors (TGA2, TGA5 and TGA6) have been shown previously to be essential for the activation of promoters containing $35 \mathrm{~S}$ as-1like elements (Fode et al. 2008). These factors are also responsible for the strong activation of $P R-1_{1294} a s-1$ as revealed by complete loss of promoter activity in the tga 2 tga5 tga6 triple mutant (Figure $4.13 \mathrm{~A}$ ). Thus, it seems likely, that these factors are necessary for the recruitment of a yet unknown factor that requires the $g$ in the first position. Nevertheless, in the absence of the subclass II members, the $35 \mathrm{~S}$ as- 1 element can still repress the LS4-dependent activation pathway, which is activated when LS5 is mutated. It is concluded that other TGA factors can recruit the repressor (SNI1?) to LS5. Whereas class II TGA factors are essential for the induction of promoters that are activated by INA in an NPR1-independent way (Blanco et al. 2005; Fode et al. 2008), conflicting data have been reported concerning their role for $P R-1$ expression. The $P R$ $1_{1294}$ promoter transformed in tga2 tga5 tga6 protoplasts shows an increased constitutive expression in relation to the wildtype control (figure $4.13 \mathrm{~B}$ ), indicating that the subgroup II members are at least partially acting as transcriptional repressors in the $P R-1$ promoter context. This conclusion is consistent with increased basal $P R-1$ expression measured previously in the triple mutant (Blanco et al. 2009; Zhang et al. 2003). 

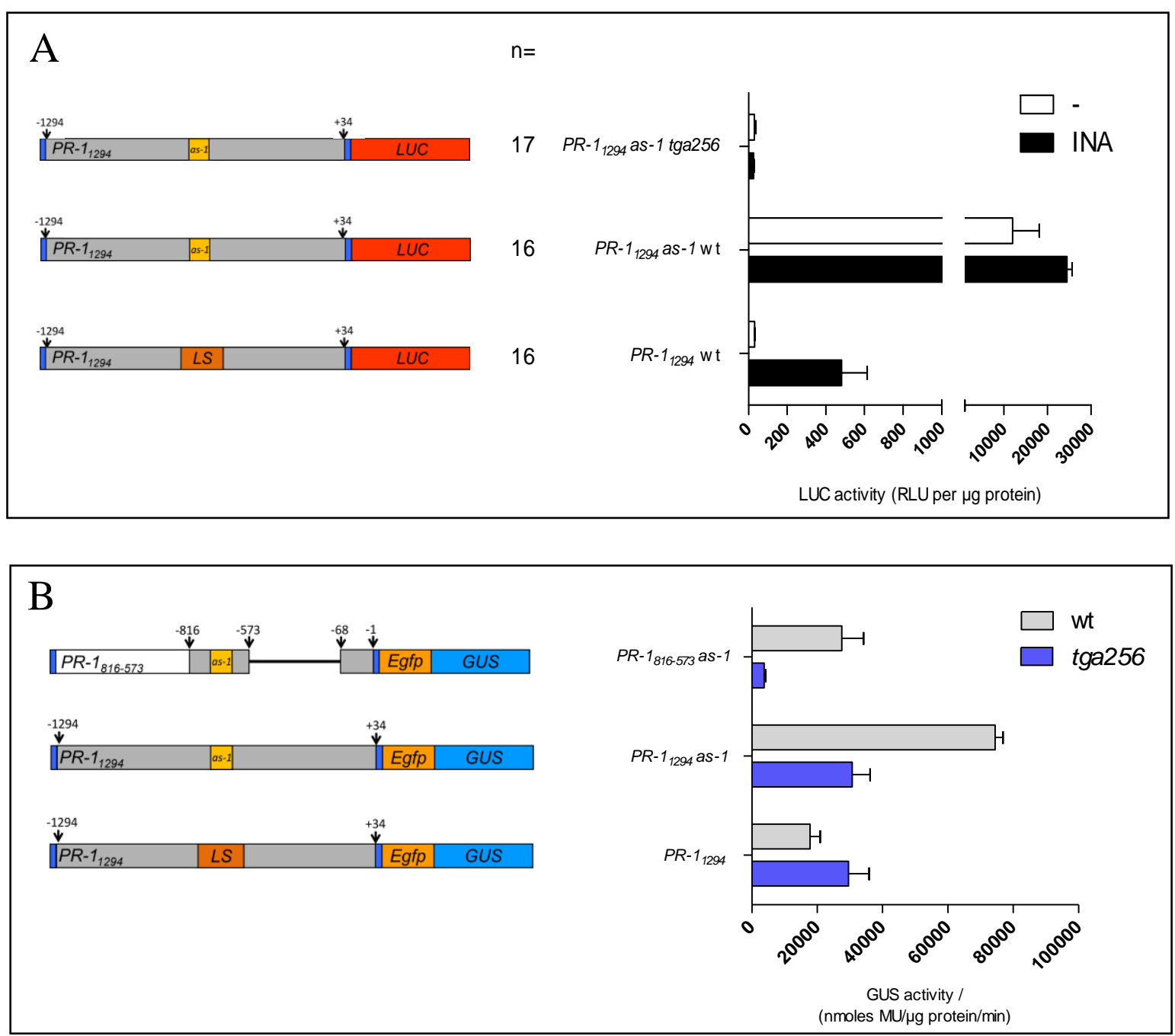

Figure 4.13: Transgenic- (A) and transient (B) expression analysis of the $P R-1_{1294}$ as-1 promoter derivative in wt and tga2 tga5 tga6.

Leaves of 3-week-old soil grown and non-induced wt and tga256 plants were used for protoplast isolation and subsequent transformation. A illustrates the activity of the $P R-1_{1294} a s-1$ reporter constructs in wt and $\operatorname{tga} 2 \operatorname{tga} 5 \operatorname{tga} 6$. The number of investigated lines is indicated $(\mathrm{n}=)$ and the error bars show the standard deviation of two independent experiments. Figure $\mathbf{B}$ shows the promoter activities of the $P R-1_{1294}, P R$ $1_{1294} a s-1$ and $P R-1_{816-573}$ as -1 constructs gained from wt- and tga 2 tga5 tga6 protoplast transformations. The ordinate displays relative GUS activity and the error bars indicate the standard deviation of four replicates. The grey columns illustrate expression levels in wt protoplasts while the blue columns correspond to the tga $2 \operatorname{tg} 55 \operatorname{tg} a 6$ mutant. The promoter illustrations on the left display the $L S$-region (orange) or a substituted $a s-1$ element (yellow). Blue boxes display the gateway® cloning sites, grey shaded regions illustrate $P R-1$ cis-regulatory regions and the white box shows sequence substitution against the coding sequence of the $C A T$ gene. Arrows and numbers indicate base pair positions. 


\subsection{In vitro binding studies reveal a similar recruitment of TGA factors to the $35 \mathrm{~S}$ as-1 - and as-1-like element}

The as-1 element substitution experiments presented so far illustrate that the composition of this regulatory unit has a severe impact on the activity of the PR-I promoter. A possible explanation for the differences in promoter activity is a differential recruitment of TGA factors to the distinct types of as-1 elements. Therefore EMSA experiments were performed to monitor the recruitment of TGA factors to the $35 \mathrm{~S}$ as- 1 element and the $a s-1$-like element derived from the $P R-1$ promoter under inducing- and non-inducing conditions.

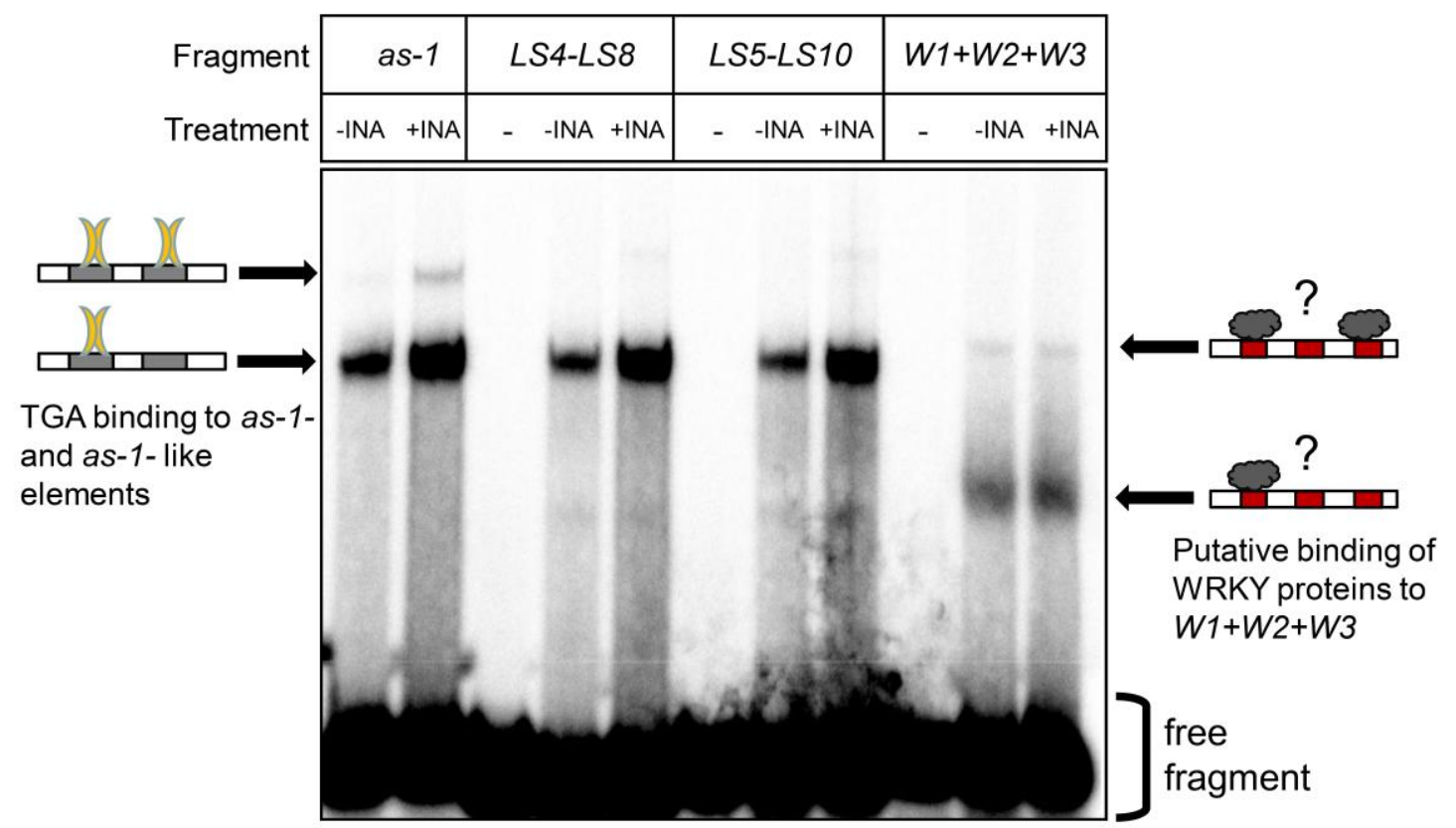

Figure 4.14: In vitro binding study of TGA factor recruitment to the $35 S$ as-1- and the PR-1 as-1like element.

The nuclear extracts used in this EMSA were obtained from 14-day-old wt plants grown on MS-medium supplemented with- or without $30 \mu \mathrm{M}$ INA. The $a s-1$ fragment is derived from the $35 S$ promoter while $L S 4-L S 8, L S 5-L S 10$ and $W 1+W 2+W 3$ contain regulatory sequences of the $P R-1$ promoter. The bolt arrows indicate single- or double occupancy of the fragments while the question marks illustrate a speculated binding of unknown proteins.

INA treated samples show an increased TGA binding affinity to the two different types of $a s-1$ elements (figure 4.14). In case of the $35 S$ as- 1 fragment, an additional band appears above the solid band illustrating recruitment of TGA homo- or heterodimers to both TGACG motifs while the $a s$-1-like element only shows a single occupancy (see 
black arrows in figure 4.14). The rightmost fragment displayed on the EMSA contains the $P R-1 \mathrm{~W}$-box cluster localized between -546 and -484 . Contrary to expectation, this fragment shows constitutive protein recruitment and no alteration when the plants were grown on INA plates. Many WRKY genes exhibit increased transcription after SA- or INA treatment; hence, a strengthened WRKY recruitment to the $W 1+W 2+W 3$ fragment would be reasonable.

The specific binding of TGA factors to the as- 1 element was proven by supershift experiments conducted with whole cell protein extracts as displayed in figure 4.15.

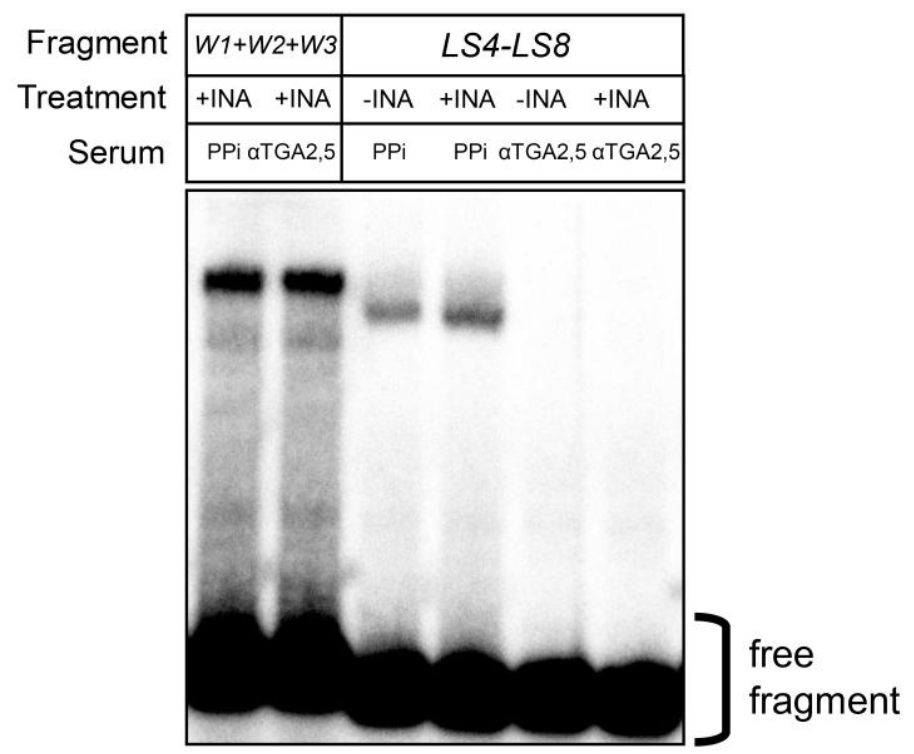

Figure 4.15: Supershift experiments performed with $\alpha$ TGA2,5 antiserum on the $W 1+W 2+W 3$ and LS4-LS8 promoter fragments.

The whole cell protein extracts were obtained from 14-day-old wt plants grown axenically on 30 $\mu \mathrm{M}$ INAor control medium plates. The $W 1+W 2+W 3$ construct serves as a negative control to show the specific effect of the serum on as-1-like element binding proteins. PPI designates addition of preimmune serum while antiserum treatment is indicated by $\alpha \mathrm{TGA} 2,5$.

The recognition of TGA transcription factors by the $\alpha$ TGA2,5 antiserum seems to inhibit the DNA binding of the bZIP proteins as no bands appear on the EMSA when the antibody is added to the samples. This loss of recruitment is specific for the as-1like element, since the W-box cluster fragment does not show an influence of the serum on general protein binding properties. Surprisingly the W-box control shows a different band pattern than observed in EMSAs performed with nuclear extracts (see figure 4.14). Though TGA association to the two different as-1-like elements occurs in a similar way it may be that differing compositions of TGA family members recruited to the regulatory sequence are responsible for the distinct impacts on global promoter activity. 
This idea was pursued by investigating TGA recruitment in several tga mutant plants (figure 4.16).

The two EMSA experiments imaged in figure 4.16 show a similar pattern of TGA factor recruitment when distinct members of the subgroup II TGA family are mutated. The tga2 tga5 tga6 triple mutant reveals that mainly the subclass II members contribute to the shifts observed in the EMSAs as just faint background signals are detectable in the SA-induced samples when all three proteins are missing. The tga6 mutant does not influence binding to the fragments while the tga2 tga5 double mutant displays a more severe effect as the protein recruitment seems to be reduced illustrated by the absence of an upper band in case of the as- 1 fragment and by general weaker signal intensities. Though there is no more in vitro TGA factor recruitment in the triple mutant, the TGACG motifs are capable to recruit other proteins or TGA family members in this mutant in vivo. As the $P R-1_{1294}$ as- 1 construct transformed in tga2 tga5 tga6 shows no expression at all the LS5-mediated suppression of the as-1-independent induction pathway is still operational.

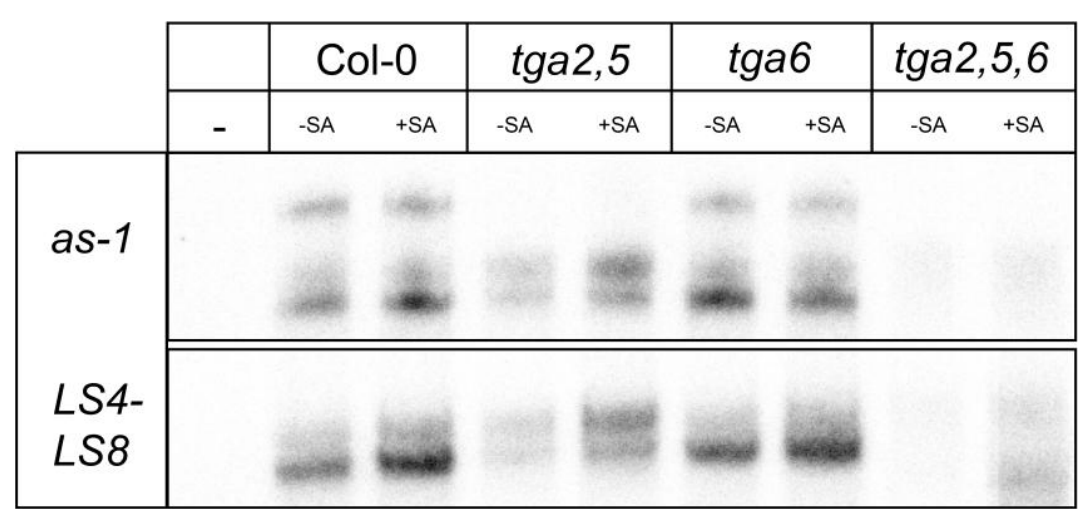

Figure 4.16: In vitro binding study of TGA factor recruitment to the two types of as-1 elements in subgroup II mutants.

The 14-day-old plants used for whole cell protein extraction were grown on MS-medium and treated with $1 \mathrm{mM}$ SA solution or $\mathrm{H}_{2} \mathrm{O} 24 \mathrm{~h}$ before harvesting the material. The figure shows two EMSA experiments exhibiting TGA recruitment to the two examined types of as- 1 elements in subgroup II tga mutants. 


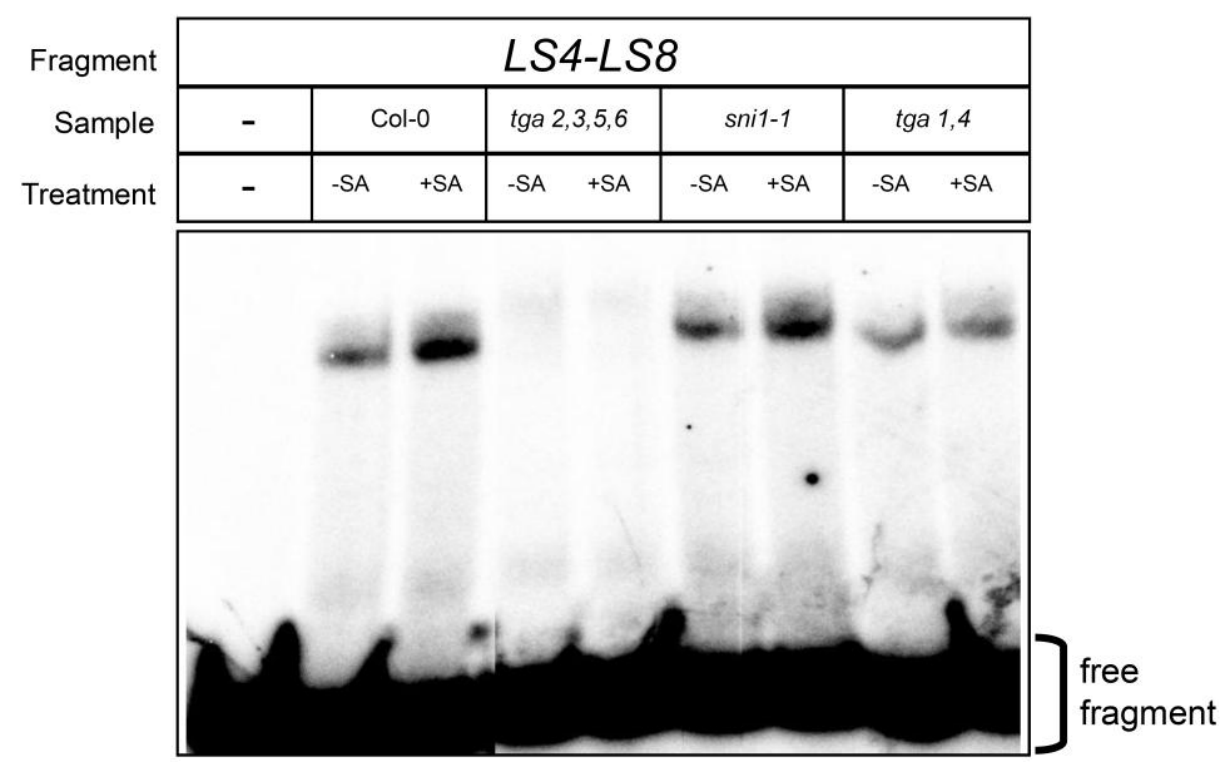

Figure 4.17: In vitro binding studies of TGA factor recruitment to the PR-1 as-1-like element in different mutant backgrounds.

Whole cell protein extracts were obtained from 14-day-old plants axenically grown on MS-plates and sprayed with $1 \mathrm{mM} \mathrm{SA}$ or $\mathrm{H}_{2} \mathrm{O} 24 \mathrm{~h}$ prior to harvest. The figure displays the TGA factor association to the LS4-LS8 fragment in tga2,3,5,6, snil-1 and tgal,4.

The gel shift experiments clearly show the redundant character of subgroup II members. The recruitment of TGA factors to as- 1 elements can at least be complemented partially when other subgroup members are absent as illustrated by the tga $2 \operatorname{tga} 5$ and the tga6 mutants.

Figure 4.17 shows an EMSA performed with the LS4-LS8 fragment and whole cell protein extracts from tga2 tga3 tga5 tga6, snil-1 and tgal tga4. While the tga quadruple mutant extracts demonstrate no TGA binding there seems to be no impact of SNI1 and the TGA subgroup I members on in vitro TGA recruitment to the as-1-like element. Thus, it seems that SNI1 does not influence promoter activity by modification of TGA factor DNA-binding affinities. 


\subsection{LS10 contributes to $P R-1$ induction in wt and snil-1}

It was reported previously that beside $L S 7$ the DOF (DNA-binding with one finger) binding motif containing element $L S 10$ shows a positive effect on $P R-1$ expression (Lebel et al. 1998). The function of LS10 in SNI1-dependent- and independent regulation was evaluated in more detail by investigating reporter gene activities of $L S 10_{\text {mut }}$ and $L S 7 / 10_{\text {mut }}$ constructs in wt and snil-1 mutants. The general inducibility of the $L S 1 O_{\text {mut }}$ promoter derivative is reduced but not abolished in wt plants (figure $\mathbf{4 . 1 8}$ A), illustrating that $L S 10$ fulfills positive functions in $P R-1$ induction. This is supported by the decreased basal- and induced expression of $L S 10_{\text {mut }}$ in snil-1. A combination of LS7 and LS10 cis-element mutations determines a complete loss of induction in both genotypes (figure 4.18 B). Since it has been reported that DOF transcription factors can stimulate TGA factor binding to $a s-1$ elements (Chen et al. 1996) it is reasonable that $L S 10$ is functioning by modulating TGA factor recruitment to $L S 7$. Taken together, $L S 7$ and $L S 10$ display additive effects as $L S 7 / 10_{\text {mut }}$ shows a more severe impact on $P R-1$ expression than the single-mutated promoters and are therefore of universal importance for the induction of $P R-1$. 

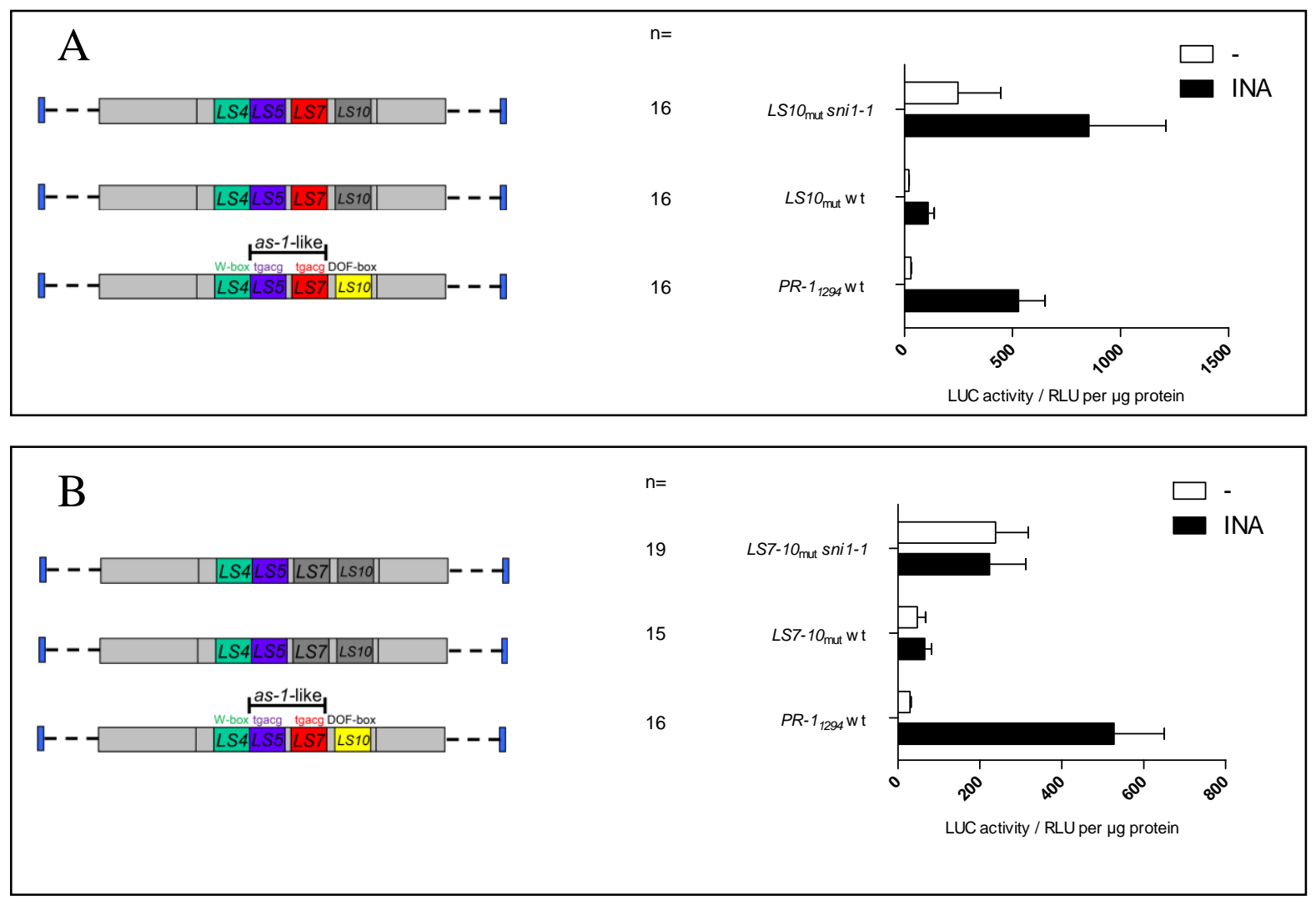

Figure 4.18: Relative average $L U C$ activities of the promoter constructs $L S 10_{\mathrm{mut}}$ and $L S 7 / 10_{\mathrm{mut}}$ in wt and snil-1.

The figure 4.18 illustrates the LUC activities of 18-day-old plants axenically grown on MS-plates supplemented with- or without $30 \mu \mathrm{M}$ INA. Figure A shows the expression of $L S 10_{\text {mut }}$ in the different genotypes while B displays the combination of both promoter element substitutions $\left(L S 7 / 10_{\text {mut }}\right)$ in wt and snil-1. The number of lines investigated for calculation of the average LUC activities is indicated $(\mathrm{n}=)$, the error bars represent two independent experiments. The dark grey shaded boxes in the promoter illustrations display 10bp mutations in the corresponding cis-elements. 


\subsection{The cis-elements $L S 4$ and $L S 10$ contribute to the overall overexpression competence of the substituted $35 \mathrm{~S}$ as-1 element}

The constitutive activation of the $P R-1_{1294} a s-1$ promoter is obviously dependent on the recruitment of an additional activator which may localize to the linker sequence of the substituted as-1 element via interaction with subgroup II TGA factors. The conclusion that the as-1 element enables constitutive expression is not consistent with previously published data as an as-1 element containing minimal $35 \mathrm{~S}$ promoter does not show increased basal activity (Redman et al. 2002). Instead the as-1 element is thought to be critical for SA- and auxin sensitivity. It seems that the strong overexpression determined by the $35 S$ as- 1 element in the $P R-1$ promoter context is dependent on surrounding regulatory sequences which are capable to boost transcriptional activity synergistically. For instance the overexpression capacity as well as the inducibility of $35 \mathrm{~S}$ as-1 substituted $P R-1$ promoter constructs is partially dependent on the W-box cluster downstream of the $L S$-region (figure 4.9). The influence of regulatory elements adjacent to the as-1 element was examined by combining LS4- and LS10 mutations with $35 S$ as1 element substitutions (figure 4.19). Mutations in the W-box $\left(L S 4_{\text {mut }}\right)$ or the putative DOF transcription factor recruitment site $\left(L S 10_{\text {mut }}\right)$ cause decreased $35 S$ as- 1 element mediated reporter gene activities. The reduction in activity is not very strong and the general character of the overexpression inducing $35 \mathrm{~S}$ as-1 element is not changed. In conclusion, it seems that the cis-elements LS4 and LS10 act as positive enhancers for the constitutive expression conditioned by the $35 S$ as- 1 element, though there are maybe other regulatory sequences which contribute to the increased transcriptional activity. 

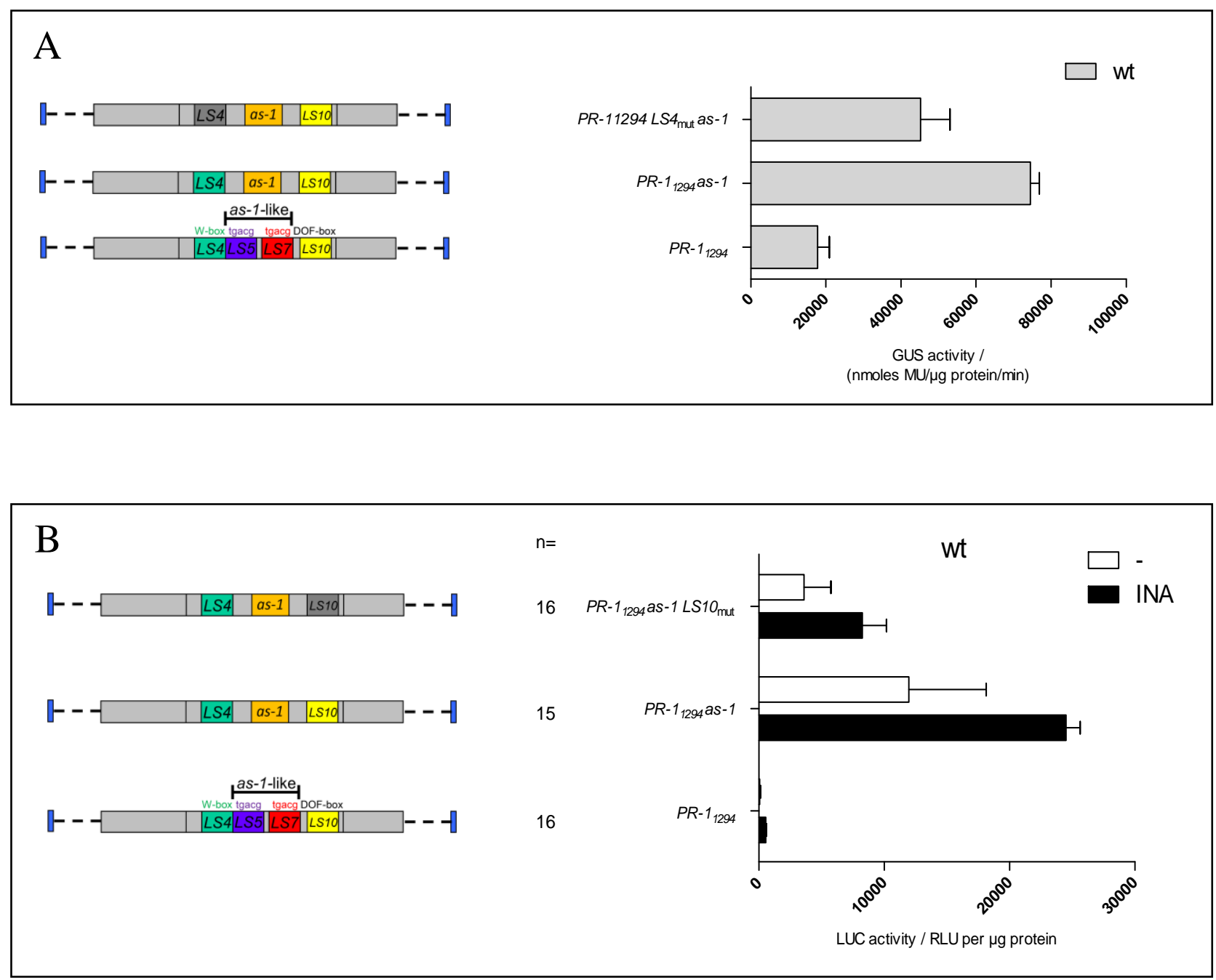

Figure 4.19: Transient- (A) and transgenic (B) expression analysis of the $P R-1_{1294} L S 4_{\text {mut }}$ as-1 and $P R-1_{1294}$ as 1 LS10 10 promoter derivatives.

Transient transformations were performed with isolated leaf protoplasts from 3-week-old soil grown and non-induced wt and tga256 plants (A). Each error bar represents the standard deviation of 4 replicates. The transgenic lines illustrated in $(\mathbf{B})$ were grown on MS-plates supplemented with- or without $30 \mu \mathrm{M}$ INA. The number of investigated single lines is indicated $(\mathrm{n}=)$, the error bars show the standard deviation of two independent experiments. A magnified view on the LS-region is shown in the promoter illustrations. The PR-1 promoter illustrations show the different sequence alterations tested in tThe $35 \mathrm{~S}$ as- 1 element is colored yellow, $10 \mathrm{bp}$ linker scan mutations are indicated by the dark grey shaded boxes. 


\section{Discussion}

The salicylic acid-inducible $P R-1$ gene serves as a marker gene that is subject to the same regulatory mechanisms that control systemic acquired resistance. Therefore, analysis of the molecular mechanisms regulating this gene is of major importance for understanding plant defense responses. Classical promoter deletion and linker scanning analysis have previously identified critical cis regulatory sequences within the so called $L S$-region of the promoter (Lebel et al. 1998). Complementary genetic approaches have led to the identification of the trans-regulatory factors NPR1, SNI1 and TGA (Cao et al. 1994; Kesarwani et al. 2007; Mosher et al. 2006; Zhang et al. 2003) The study presented here combines both approaches by analyzing distinct $P R-1$ promoter derivatives in different mutant backgrounds implicated in SA signaling and $P R-1$ regulation. This strategy yielded the following novel information: (1) The promoter contains a W-box cluster downstream of the $L S$-region which contributes to $P R-1$ induction and hyperinducibility in snil-1. (2) The corresponding WRKY transcription factors are regulated in an NPR1/SNI1-dependent manner (3) The as-1-like element presumably serves as direct target site for TGA/NPR1 mediated regulation. (4) NPR1dependent regulation at $a s-1$ elements is only operational when the distance between the TGACG-motifs is not too close. (5) The as-1-like element is not essential for $P R-1$ induction, as an alternative pathway can be activated in the absence of the TGACG motif LS5. (5) A TGA/SNI1 complex is potentially formed at the LS5 site to suppress the as-1-independent pathway. (6) Induction of the as-1-independent mechanism is influenced by WRKY factors whose regulation is dependent on NPR1 and SNI1. (7) The sequence between the palindromic repeats determines the recruitment of transcriptional activators or repressors. The data obtained in this study give rise to detailed models of $P R-1$ regulation.

One of the most unexpected results of this work is the finding that the as-1-like element, which has been considered as the crucial binding site for NPR1-dependent regulation of the promoter, is dispensable for induction ( $L S 5 / 7$ mut, figure 4.3). In the absence of this element, an alternative pathway can be activated. The function of the different cis elements of the $L S$-region, which has been found previously to contain critical cis 
elements (Lebel et al. 1998), and the W-box cluster between -573 and -486 , is discussed with respect to their functional relevance for the two pathways.

\subsection{A W-box cluster enhances $P R$ - 1 expression and integrates signals of SNI1-repressed activators}

A novel regulatory region identified in this study is the W-box cluster located between 573 and -486 relative to the transcriptional initiation site. When compared to the wildtype promoter, the $\mathrm{W}$-box cluster-lacking $P R-1_{816-573}$ construct shows reduced transcription after INA treatment, indicating that it contributes to maximal expression, but that it is not essential. However, these W-boxes are required for the strong hyperinduction of the promoter in snil-1 (figure 4.2 B and 4.7). As hyperinducibility depends on the as-1-element in the $L S$-region (see figure 4.4 and below), we speculate that the TGA/NPR1 complex synergistically interacts with WRKY factors at the downstream W-boxes.

WRKY proteins are members of a large class of over 70 DNA-binding proteins in Arabidopsis (Eulgem and Somssich 2007) that can either positively or negatively affect transcription of either pathogen- or senescence-regulated genes. The function of individual members of this family has been addressed by analyzing knock-out mutants and plants with ectopic expression. Ectopic expression of WRKY70 yields constitutive activation of $P R-2$ and $P R-5$ (Li et al. 2004). In the case of $P R-1$, an aberrantly long mRNA appears upon mock treatment, but the SA-regulated accumulation of the wildtype transcript is not altered. All three genes usually show a similar expression pattern during SAR or after SA treatment and are therefore grouped in the same regulon (Maleck et al. 2000). One main difference between the cis-regulatory sequences of these genes is the absence of an $a s-1$-like element in case of $P R-2$ and $P R-5$. As a number of evidences of this study point at the $a s$-1-like element functioning as an indirect target site for SNI1, we speculate that the $P R-1$ promoter is in a "locked" state, where WRKY factors cannot achieve any activation, unless SNI1 is counteracted by activated NPR1. In contrast, $P R-2$ and $P R-5$, which are presumably not under the direct control of SNI1, can be activated by ectopically expressed WRKY factors. 
Interestingly, ectopic expression of WRKY18 can lead to constitutive PR-1 expression, which is dependent on NPR1. However, this constitutive expression only occurs in at least 4 week-old plants. Nuclear localized NPR1 may accumulates in older leaves and this accumulation could be sufficient to interfere with SNI1 action so that activation of $P R-1$ by WRKY18 can occur in the absence of INA.

One approach to investigate regulated events at the W-box cluster was undertaken by EMSA experiments to monitor the protein recruitment to the $P R-1 \mathrm{~W}$-box cluster under non-inducing and inducing conditions (figure 4.14 and 4.15). Surprisingly, the $W 1+W 2+W 3$ fragment showed constitutive protein binding instead of an inducible shift, though other groups reported altered protein recruitment to similar W-box fragments under non- and SA-induced conditions (Dong et al. 2003; Knoth and Eulgem 2008). An explanation for the lacking inducible shift may be that our EMSA conditions or the protocol used for protein extraction are not capable to reveal the protein recruitment occurring at the $\mathrm{W}$-boxes in planta. Additionally, the gel shifts revealed that different proteins are recruited to $W 1+W 2+W 3$ as EMSAs conducted with nuclear extracts show a distinct band pattern than EMSAs performed with whole cell extracts (compare figure 4.14 and 4.15).

\subsection{The $P R-1$ promoter is regulated by an as-1-dependent- and as- 1 -independent induction pathway}

In previous studies, the 1294 bp $P R-1$ promoter with a mutated LS5 element showed slightly increased basal and induced expression levels (Lebel et al. 1998). Therefore, it was concluded that the LS5 element is a negative-acting TGA recruitment. Increased expression levels in $L S 5_{\text {mut }}$ lines could not be detected in our experiments, but the negative function of this element became evident after analysis of the $L S 5 / 7_{\text {mut }}$ construct (figure 4.3): While mutation of the more downstream located TGACG motif (LS7) causes a considerable decrease in reporter activity, the additional mutation of LS5 restores induction. It is concluded that $L S 5$ represses a pathway that is independent from LS5 and LS7 and which we call the as-1-independent pathway. Mutation of LS5 and the adjacent W-box $L S 4$ ( $L S 4 / 5_{\text {mut }}$ ) leads to an abolished expression under inducing conditions (figure 4.3). This result indicates that the W-box LS4 is essential for the as-1- 
independent pathway. As $L S 4$ is dispensable for the as-1-dependent pathway (figure 4.3), this construct reveals that $L S 5$ is required for the $a s-1$-dependent pathway.

\subsection{The as-1-independent pathway requires NPR1}

Analysis of the transgenic $L S 5 / 7_{\text {mut }}$ lines revealed that NPR1 is still essential for the induction of this promoter construct. At first glance, this seemed unexpected, as the as1-like element has been hypothesized before to function as the recruitment site for NPR1. However, a number of NPR1-dependent genes, like for instance $P R-2$ and $P R-5$ do not contain a TGACG motif, indicating that NPR1 might either control gene activity indirectly through the regulation of e.g. WRKY transcription factors, which contain TGACG motifs and are therefore under direct control of NPR1 (Dong et al. 2003; Wang et al. 2006). This seems likely, as the as-1-independent pathway depends on LS4, which might recruit such SA-inducible, NPR1-dependent WRKY transcription factors. Alternatively other proteins might be able to recruit NPR1 to the $L S 5 / 7_{\text {mut }}$ promoter. Recruitment of NPR1 to the $P R-1$ promoter has been detected by chromatin immunoprecipitation experiments in the tga2 tga5 tga6 mutant, which lacks the so called subclass II TGA factors (Rochon et al. 2006). However, other TGA factors may complement the function of the subclass II members. In addition, no conserved TL1 motifs, associated with NPR1-mediated regulation of genes involved in the secretory pathway (Wang et al. 2005), are present in the $P R-1$ promoter. We consider it most likely that INA-activated NPR1 induces transcription of WRKY factors, which are in turn binding to LS4 and possibly the W-box cluster, thus leading to the up-regulation of $P R-1 . P R-1$ is an unusually complex promoter as it can be under direct and indirect regulation of NPR1. The as-1-element provides the promoter with the advantage of a tighter regulation as revealed by the decreases background levels of the $P R 1_{1294}$ promoter as compared to the $L S 5 / 7_{\text {mut }}$ promoter. 


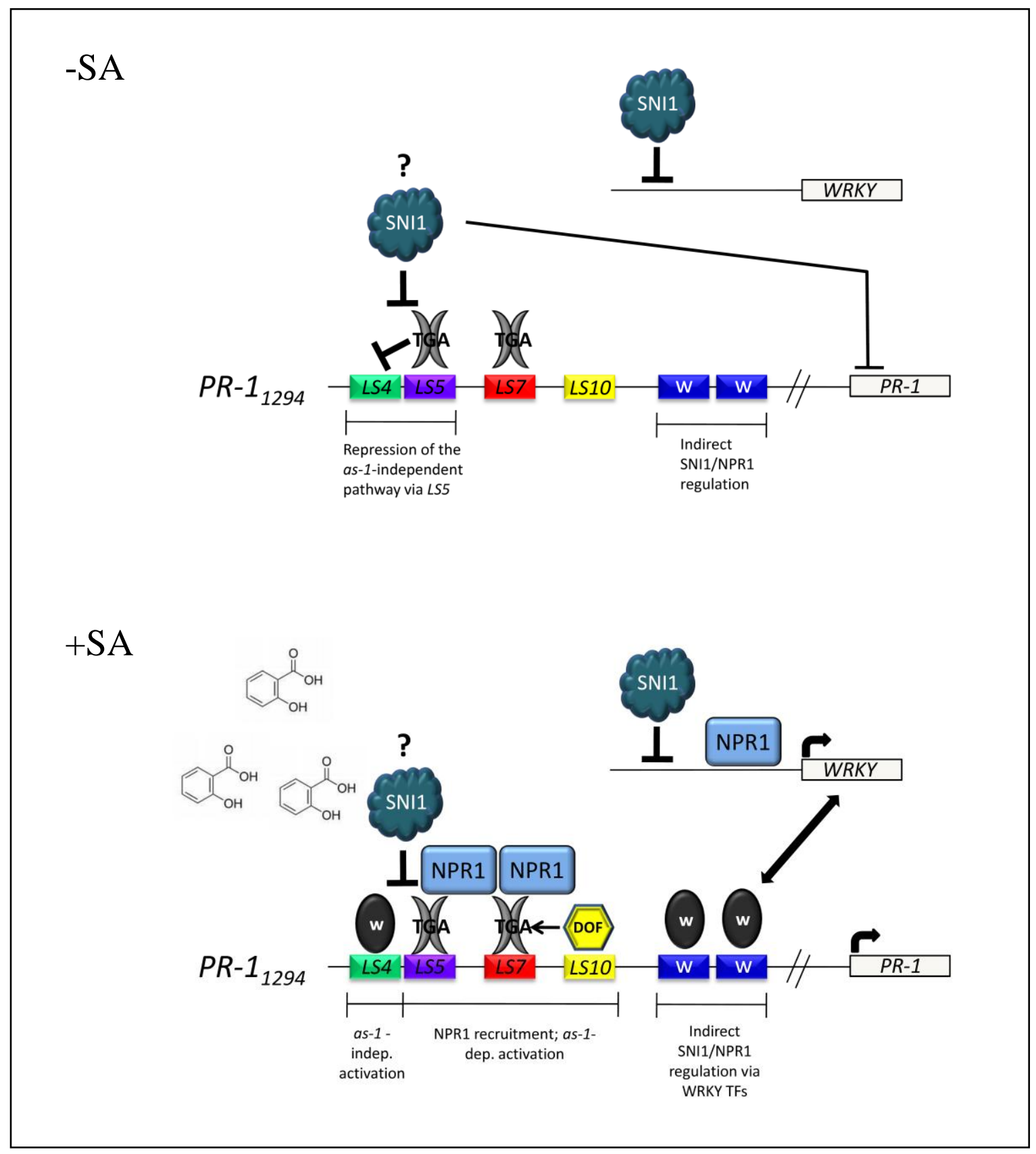

Figure 5.1: Model of $P R-1$ regulation under non-inducing- and inducing condition in wt.

SNI1 represses the $P R-1$ promoter via the $a s-1$-like element and indirectly via repression of WRKY TFs. When SA levels rise, NPR1 is shuttled to the nucleus and interacts with TGA TFs whose DNA binding affinity is increased under these conditions. NPR1 also induces WRKY TFs which serve as additional activators for $P R-1$ induction. The $a s-1$-independent induction pathway ( $L S 4)$ is constitutively repressed via $L S 5$. A putative SA-inducible DOF transcription factor enhances TGA binding to the promoter. 


\subsection{SNI1 might be the repressor acting at LS5 to repress the as-1- independent pathway}

One hypothesis to explain the negative effect of $L S 5$ on the as-1-independent transcription is that SNI1 is responsible for this effect. Consistent with this idea is that the as-1-independent pathway shows increased basal expression levels, a phenomenon also observed in snil-1. Supporting data for this assumption were obtained from reporter gene constructs harboring the two original TGACG motifs LS5 and LS7 but an altered distance between the centers of the palindromic repeats (LS5aaaaLS7). This promoter showed only very weak sensitivity to INA, which indicates that repression of that as-1-independent pathway was operational. In contrast, the as-1-dependent pathway, which depends on the direct recruitment of NPR1 to the TGA factors at the as-1-like element, did not seem to function (figure 4.8). Intriguingly, the LS5aaaaLS7 construct is active in snil-1 illustrating that SNI1 could be the repressor of this construct. The induction of LS5aaaaLS7 is not as strong as the PR- $1_{1294}$ control in snil1. This is consistent with the observation made with the $L S 5 / 7_{\text {mut }}$ promoter (figure 4.4) that a functional as-1-element is required for hyperinduction of the $P R-1$ promoter in the presence of INA.

\subsection{LS4 is a positive element in the absence, and a negative element in the presence of $L S 5$}

The W-box LS4 was previously reported to be a negatively acting cis-element as mutation of this box yielded an approximately 4-fold increased reporter gene expression in wt after INA induction (Lebel et al. 1998). This result led to the hypothesis that SNI1 regulation may functions via WRKY transcription factor interaction to suppress the activity of the promoter (Kesarwani et al. 2007). Though experiments conducted with $L S 4_{\text {mut }}$ constructs presented in this work also showed an approximately 2-fold increased average expression level as compared to $P R-1_{1294}$ control plants, combinatorial promoter sequence mutations ( $L S 4 / 5_{\text {mut }}$ and $L S 4 / 7_{\text {mut }}$; figure 4.6) clearly revealed an additional positive function of this element concerning $P R-1$ induction by providing an as-1 independent activation mechanism. The factor recruited to $L S 4$ usually mediates a weak suppression on the as-1-dependent pathway, but mutation of the TGACG motifs 
turns the character of $L S 4$ from a weak silencing- to a critical enhancing cis-element.

Since WRKY transcription factors can act as transcriptional activators or repressors (Eulgem and Somssich 2007), the conversion of LS4 function maybe associated with the recruitment of different WRKY transcription factors in case of a present- or lacking as1 -like element. For instance the endogenous $P R-1$ promoter may recruits WRKY38 and/or WRKY62 to LS4, two SA-inducible WRKY TFs known to be negative regulators of $P R-1$ expression (Kim et al. 2008). When the TGA binding sites are mutated $\left(L S 5 / 7_{\text {mut }}\right)$ binding affinity for a positive-acting WRKY to $L S 4$ could be increased (e.g. WRKY18 or WRKY70) and the $a s$-1-independent induction pathway is established. In addition, the $L S 4_{\text {mut }}$ construct was examined in $n p r 1-1$ (see supplemental data; table 6.3.1) to rule out the possibility that mutation of the putative SNI1 recruitment site leads to NPR1-independence, similar to the events in the sn1 nprl double mutant. As expected, no increased reporter gene activities were detected when these lines were grown on INA plates. In summary, it is unlikely that LS4 is the recruitment site for SNI1 but it contains a putative binding site for a positive regulator which mediates as-1-independent induction if repression through LS5 is released.

\subsection{The LS7 element is essential for PR-1 induction in snil-1}

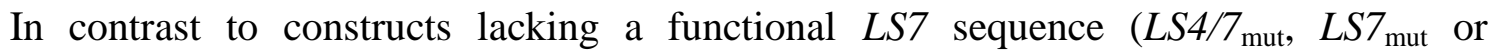
$\left.L S 7 / 10_{\text {mut }}\right)$ the inducibility of $L S 4 / 5_{\text {mut }}$ was restored in snil-1 illustrating that a nonmutated LS7 is needed for induction in this mutant. However, two issues have to be considered: (1) Mutation of one or both TGACG motifs is always associated with an increased basal expression in snil-1 similar to endogenous $P R-1$ expression in the tga 2 snil-1 double mutant (Kesarwani et al. 2007). (2) This background expression makes it hard to distinguish if a promoter is "not inducible" or "completely de-repressed". For instance, the $L S 7_{\text {mut }}$ construct shows a very strong constitutive expression in snil-1 (figure 4.4) but hyperinduction cannot occur as NPR1-dependent regulation is disturbed; hence, the promoter shows no inducibility. In case of the $L S 4 / 5_{\text {mut }}$ construct the basal activity in snil-1 is rather moderate (figure 4.6) and the functional LS7 element is sufficient for a 4 -fold induction. The activation of the $L S 4 / 5_{\text {mut }}$ construct is maybe also repressed as SNI1 is constitutively repressing the promoter via LS7. Interestingly, LS7 seems to mediate activation of this construct in snil-1 though LS5 is mutated and the as-1-dependent pathway should be dysfunctional. Thus, it is concluded 
that induction of the $L S 4 / 5_{\text {mut }}$ construct in snil-1 is achieved by a synergistic activation process mediated by a putative TGA/NPR1 complex at $L S 7$ and deregulated WRKY factors functioning via the $\mathrm{W}$-box cluster. The importance of the $\mathrm{W}$-box cluster has been shown by analysis of the $L S 4 / 5_{\text {mut }} \Delta W 1-3$ construct in this context, which did not display sensitivity to INA in snil-1(figure 4.7).

\section{$5.7 \quad L S 7$ and $L S 10$ are potentially both involved in TGA factor recruitment and NPR1 mediated activation}

The TGACG motif containing element $L S 7$ shows a general positive effect on $P R-1$ regulation in wt since $L S 7_{\text {mut }}$ causes a severe loss of induced expression. The influence of $L S 7$ on promoter activity is not as strong in our experiments as previously described by Lebel et al. who reported a completely abolished sensitivity to INA when $L S 7$ was mutated. The same holds true for a construct harboring a mutated DOF transcription factor binding site $\left(\mathrm{LS}_{\mathrm{O}} \mathrm{mut}_{\mathrm{m}}\right)$ which still exhibits weak inducibility in wt plants in our hands. Mutation of both elements $\left(L S 7 / 10_{\text {mut }}\right)$ finally provokes an absolute insensitivity to INA treatment in wt and snil-1. These results show that $L S 7$ and $L S 10$ are implicated in general activation processes as induction also fails when the repressor SNI1 is absent. Both elements probably influence expression in a synergistic way as it is reported that the DOF transcription factor OBP1 can enhance TGA4 recruitment to the sequences of as-1 elements in vitro (Chen et al. 1996). The supporting function of OBP1 is dependent on DOF recruitment sites adjacent to the $a s-1$ elements of the $35 S$ - and GST6 promoters. In case of the PR-1 promoter this increased binding might enables efficient NPR1 recruitment. The weak inducibility of $L S 7_{\mathrm{mut}^{-}}$and $L S 10_{\text {mut }}$ lines potentially originates from a slightly derepressed $a s$-1-independent pathway, caused by declined repressor recruitment to the $a s-1$-like element or $L S 5$ respectively. As $L S 7$ only has a presumably moderate impact on the repression of the as-1-independent pathway, the induction is considerably decreased in relation to the $L S 5 / 7_{\text {mut }}$ construct. However, the mechanism occurring at the $L S 10$ element to increase $P R-1$ expression remains elusive as a proof for an in vivo implication of DOF/TGA-interactions regarding gene expression has not been reported yet. 


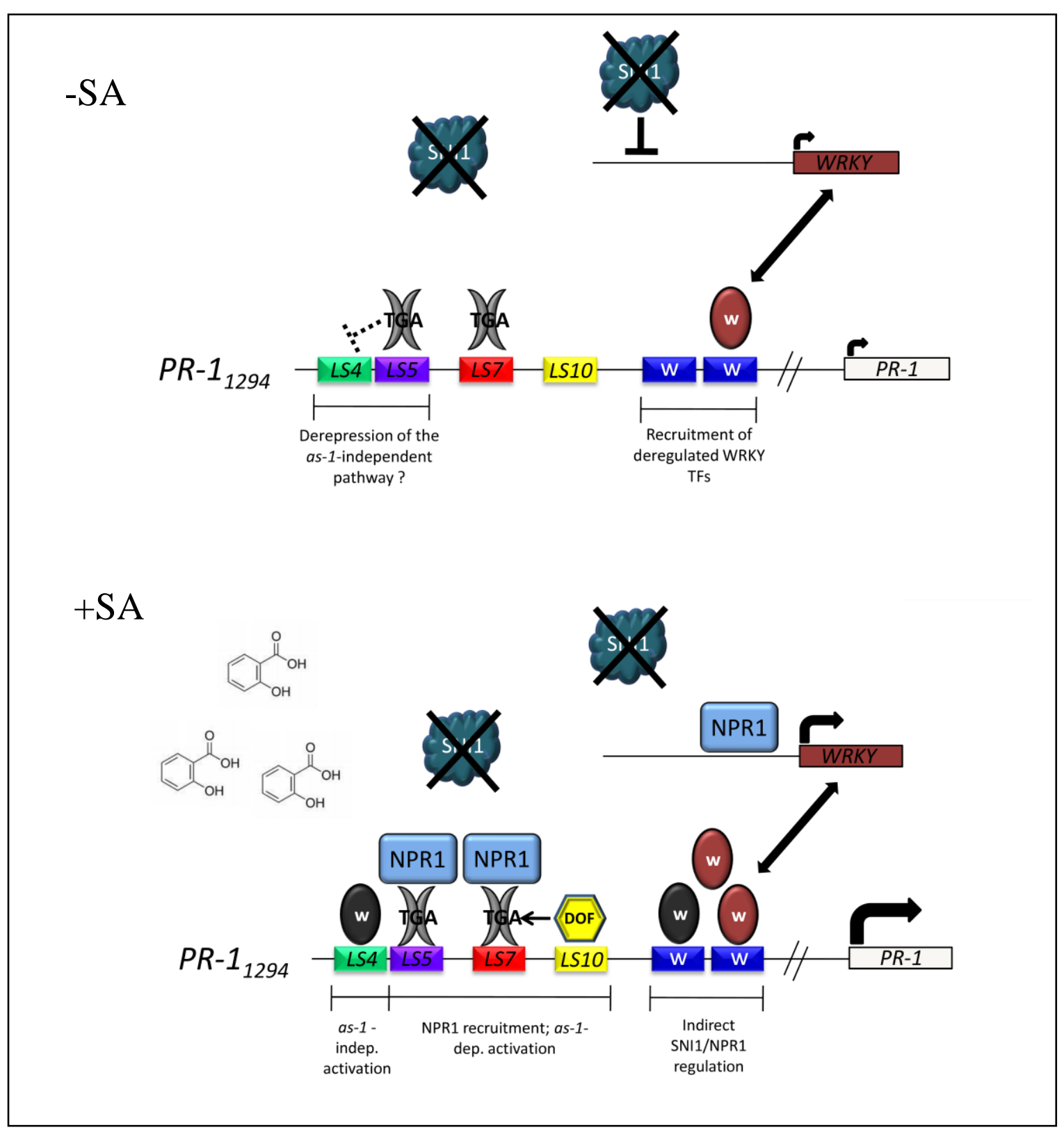

Figure 5.2: $P R-1$ regulation under inducing- and non-inducing conditions in snil-1.

Due to the absence of the repressor SNI1 the expression of WRKY genes is deregulated and the putative direct suppression via the as-1-like element is reduced leading to an increased basal expression. After induction treatment the NPR1-mediated regulation at the as-1-like element and the regulation at the more downstream located $\mathrm{W}$-box cluster act synergistically to induce hyperinduction of $P R-1$. 


\subsection{PR-1 regulation in snil-1 and snil nprl mutant background}

Figure 5.2 displays our model for $P R-1$ regulation in snil-1 mutant background. Mutation of SNI1 leads to derepression of positive regulators involved in defenserelated processes, e.g. distinct WRKY transcription factors (Mosher et al. 2006). These de-regulated WRKY factors induce the strong expression levels monitored in snil-1 via the W-box cluster between -573 and -486 as revealed by the analysis of the $P R-1_{816-573}$ construct in comparison to $P R-1_{816-509} W 1+W 2$ (figure 4.2 B). The W-box cluster does not only boost signal intensities in snil-1, it is also essential for NPR1-independent activation of the promoter as the $\mathrm{W}$-box cluster lacking $P R-1_{816-573}$ constructs shows no induction in the snil nprl double mutant (figure 4.1 B). Beside the W-box cluster direct NPR1-dependent regulation via the as-1-like element contributes to the strong induction in sni1-1, since mutations in LS5 and/or LS7 lead to weaker reporter gene activities. Furthermore, endogenous $P R-1$ induction in snil nprl is decreased in relation to the snil-1 mutant. At the genetic level, PR-1 induction in snil nprl is dependent on RAD51D, a recombination factor identified in a mutagenesis screen for a rescued snil nprl leaf phenotype (Durrant et al. 2007). Mutation of RAD51D completely abolishes PR-1 induction in the rad51d snil nprl triple mutant while the rad51d snil double mutant lacks the increased $P R-1$ background expression and hyperinducibility. The wrinkled leaf phenotype conditioned by the snil-1 mutation is reverted to wildtype leaf morphology in both mutants. These results indicate that SNI1-repressed genes are positively regulated by RAD51D, but this positive effect can only be monitored in snil1 mutant plants, as rad51d single mutants neither show an impact on $P R-1$ expression nor on the leaf phenotype. Concerning our model RAD51D maybe induces expression of the deregulated WRKY transcription factors in snil-1 and thereby contributes to the hyperinduction of $P R-1$ in this mutant and to the general inducibility of the snil nprl double mutant via W-box cluster-mediated activation. 


\subsection{An activator is recruited to the $35 S$ as- 1 element in the $P R-1$ promoter context}

Substitution of the as-1-like- against the $35 S$ as- 1 element yields a substantial increase of gene expression under uninduced- ( 400-fold) and induced ( $\sim 50$-fold) conditions in wildtype plants (figure 4.8). The strong activation potential of the $35 \mathrm{~S}$ as-1 element is strictly dependent upon subclass II TGA factors (figure 4.13 A) and the linker sequence "ggga" between the TGACG motifs (figure 4.12). NPR1 is no longer required to activate the promoter but it is still able to modulate transcription as overall expression levels are lower in nprl-1 than in wt and snil-1 mutant plants (figure 4.8). The influence of the regulator NPR1 and the inducibility are vanished in the $P R-1_{816-573}$ as- 1 construct illustrating that processes at the W-box cluster are maybe responsible for the induction and increased background expression detected with the full length $P R-1_{1294}$ as-1 promoter derivative.

In conclusion, the $35 \mathrm{~S}$ as-1 element recruits a strong constitutive activator to its linker sequence via TGA subclass II transcription factors but does not assure autonomous INA-sensitivity in the $P R-1$ promoter context (figure 5.3).

Potential additional proteins recruited to the $35 \mathrm{~S}$ as-1 element belong to the classes of Myb- and NAC transcription factors, as putative binding sites for these proteins are encoded in the sequence (Baranowskij et al. 1994; Duval et al. 2002). It has been reported that the potato Myb-like protein MybSt1 specifically binds to the "ggatg" sequence of the as-1 element and activates transcription (Baranowskij et al. 1994). Though the "ggatg" motif is changed to "ggatt" in the constitutive active LS5gggaLS7 derivative (figure 4.10 A) recruitment of a Myb factor cannot be excluded as Baranowskij et al. showed that a perfect binding site for MybSt1 ("ggata") displayed 20-30 fold higher binding affinity than the as-1 sequence. This result shows that the most downstream located guanine in the "ggatg" binding site does not necessarily increase Myb recruitment. BLAST search revealed that there are several Arabidopsis proteins ( 16) sharing a high protein sequence homology to MybStl. The gene with the highest sequence similarity (At5g47390) shows a constitutive expression in leaf tissue and additionally no sensitivity to the plant hormones SA, JA, GA and ABA (Yanhui et al. 2006). Therefore, this so far non-characterized Myb factor is a good candidate for 
mediating constitutive expression of the $P R-1_{1294} a s-1$ construct via interaction with subclass II TGA factors.

Another transcriptional activator known to bind to the $35 \mathrm{~S}$ as- 1 element linker sequence is the NAC transcription factor AtNAM as revealed by DNase I footprinting (Duval et al. 2002). The region protected from DNase I digestion in this assay included the nucleotide sequence "gggatg" and thereby contains the putative MybSt1 binding site. In contrast to the closest MybSt1 ortholog in Arabidopsis (At5g47390) AtNAM is exclusively expressed in developing- and mature seeds. Therefore, it is questionable if AtNAM could induce such strong expression levels monitored with $P R-1_{1294}$ as -1 promoter constructs in leaf tissue. However, the NAC family of transcription factors consists of 106 members ( $\mathrm{Qu}$ and Zhu 2006) and it cannot be ruled out that other NAC factors can bind to the linker sequence of the as-1 element and activate expression. Regardless to the question if a NAC- or a Myb transcription factor is recruited to the $35 S$ as- 1 element the first guanine in the linker sequence ("ggga") is critical for the binding of the constitutive activator as revealed by transient protoplast transformations with $P R-1_{1294}$ as- 1 promoter constructs harboring mutations in the linker sequences (figure 4.12). Furthermore it could be shown that the elements $L S 4$ and LS1O positively influence constitutive induction mediated by a substituted $35 \mathrm{~S}$ as- 1 element, but the effect is only moderate (figure 4.19). 


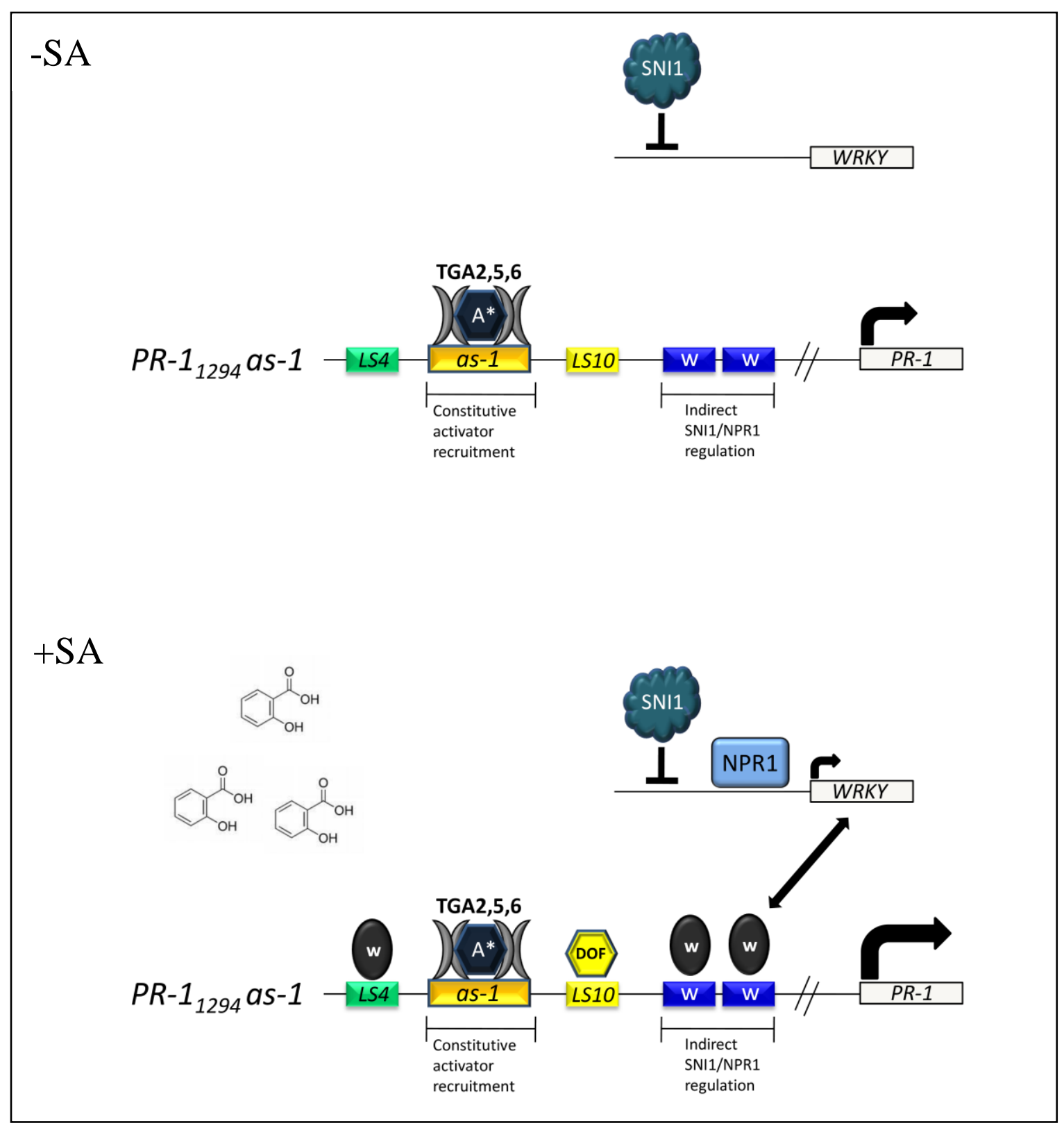

Figure 5.3: Model showing the constitutive activation of the $P R-1$ promoter by a substituted as-1 element derived from the $C a M V 35 S$ promoter in wildtype plants.

The mutated as- 1 element recruits an activating protein which strongly increases basal expression of the promoter. After SA-treatment other regulatory sequences, presumably the W-box cluster, contribute to an approximately twofold synergistic induction. The recruitment of the unknown activator is strictly dependent on subclass II TGA factors. 


\subsection{In the absence of subclass II TGA factors a negative regulator is recruited to the $35 S$ as- 1 element}

As mentioned above, mutation of the subclass II TGA factors leads to a completely abolished expression of the $P R-1_{1294} a s-1$ reporter constructs under non-inducing- and inducing conditions (figure $4.13 \mathrm{~A}$ ). This result illustrates two remarkable issues: (1) The unknown constitutive activator requires these TGA factors for $a s-1$ element binding and (2) a constitutive repressor must be recruited to the $35 \mathrm{~S}$ as- 1 element in tga 2 tga5 tga6 since the endogenous $P R-1$ promoter shows increased basal expression and inducibility in this mutant (Blanco et al. 2009). The binding of the repressor obviously depends on other TGA transcription factor family members as deletion of both TGACG motifs $\left(L S 5 / 7_{\mathrm{mut}}\right)$ regains inducibility (figure 4.3). The recruitment of the suppressor complex to $35 S$ as- 1 only occurs in the absence of TGA2, TGA5 and TGA6, likely because the subclass II TGA factor-mediated binding of the activator complex is disturbed. Induction of the $P R-1_{1294} a s-1$ construct is dysfunctional in the triple mutant as direct NPR1 regulation cannot occur at the $35 S$ as- 1 element due to the shortened distance (4bp) between the TGACG motifs. Taking into account the results obtained with the LS5aaaaLS7 construct in wt and snil-1 (figure 4.8) it is reasonable that SNI1 suppresses the as-1-independent induction pathway via the $35 S$ as- 1 element in tga 2 tga5 tga6. Unfortunately, a tga2 tga5 tga6 snil-1 quadruple mutant is not available so far to further verify the hypothesis of SNI1 recruitment to the $35 \mathrm{~S}$ as- 1 element.

The presence of a suppressor complex at the $35 \mathrm{~S}$ as-1 element in the triple mutant further indicates that EMSA binding studies do not reflect the protein recruitment to the as- 1 element occuring in planta as no gel shifts appeared when whole cell extracts from tga2 tga5 tga6 plants were used (figure 4.16).

In conclusion, the experiments conducted with the $P R-1_{1294}$ as- 1 construct in tga2 tga5 tga6 mutant plants illustrate that the $35 \mathrm{~S}$ as -1 element is a potential recruitment site for a transcriptional activator or repressor, dependent on the TGA factors available in the plant. In the presence of subclass II TGA factors activator binding to the as- 1 element is mediated whereas other TGA family members are able to recruit a repressor, presumably SNI1, in the absence of TGA2, TGA5 and TGA6. 


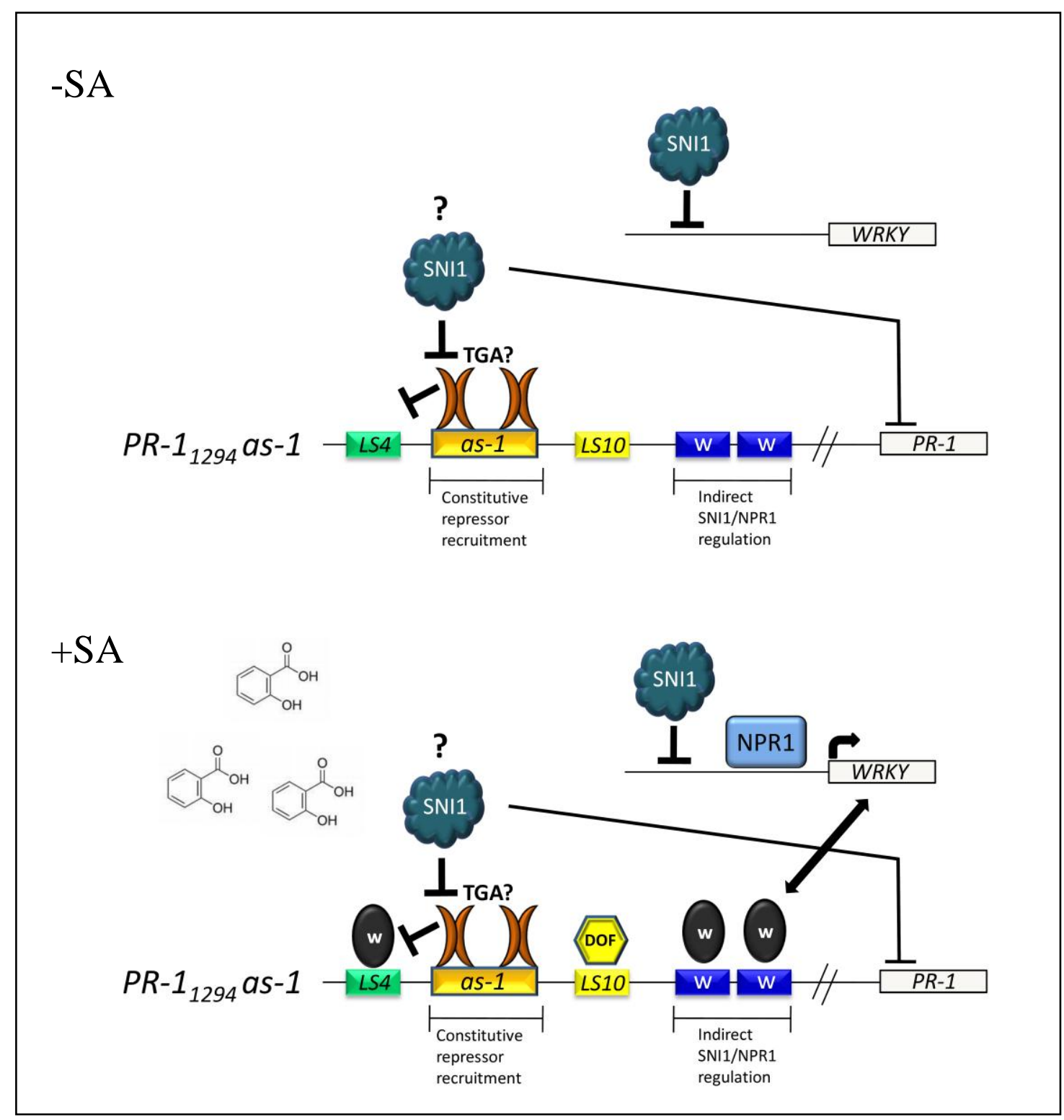

Figure 5.4: Model explaining the abolished expression of the $P R-1_{1294}$ as -1 construct in $\operatorname{tga} 2 \operatorname{tga} 5$ tga6 mutant background.

In the absence of subclass II TGA factors other TGA factors are recruited to the promoter which do not enhance activator- but repressor recruitment. A potential repressor recruited to the $a s-1$ element is SNI1 which suppresses the activation of the as- 1 -independent pathway efficiently. As the distance between the TGACG motifs is altered NPR1 can no longer be recruited to the as- 1 element and induction fails. 


\section{Supplemental data}

\subsection{Promoter constructs}

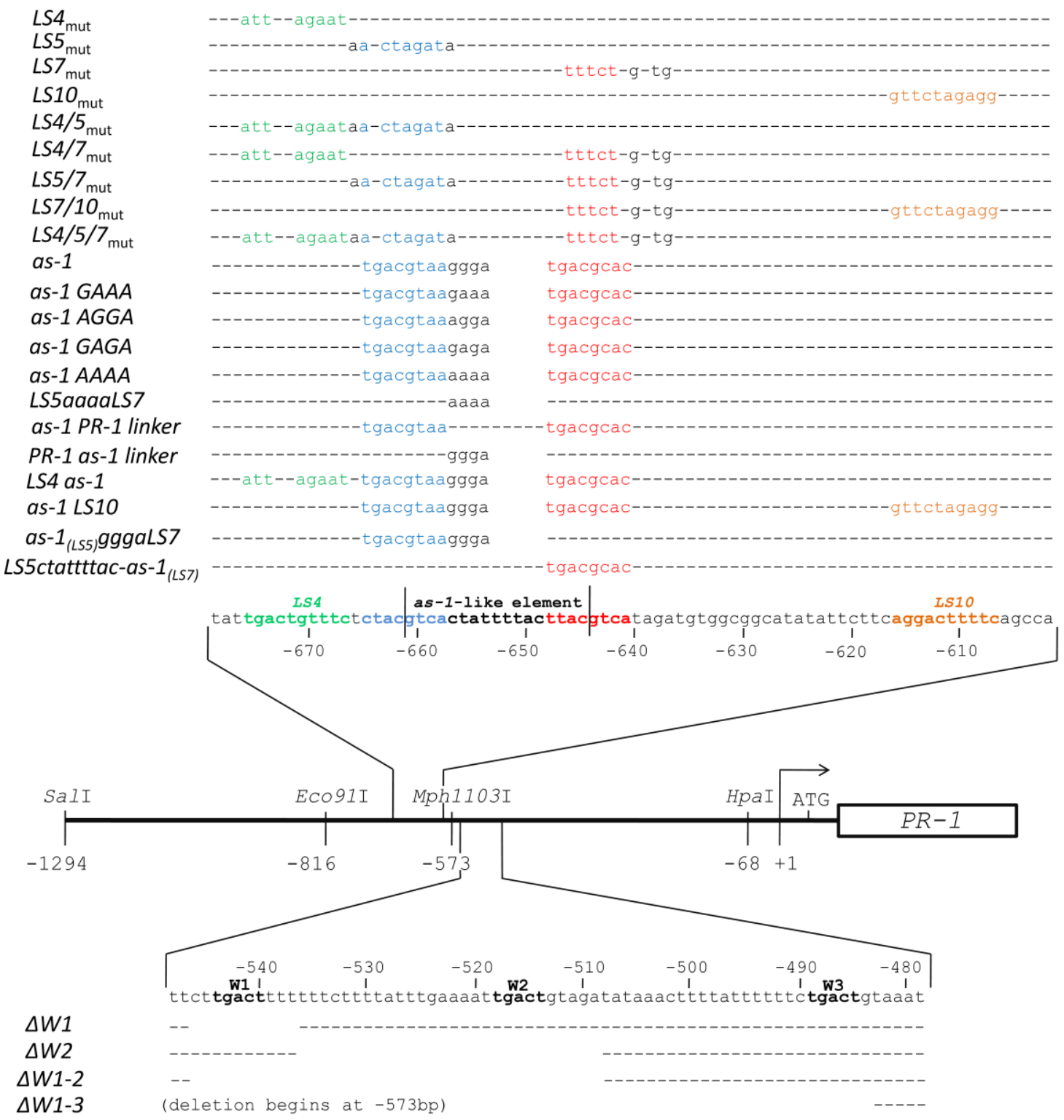

Figure 6.1: Composition of the different $P R-1$ promoter derivatives presented in this work.

On the top, distinct substitution constructs within the linker scanning region between $-602 \mathrm{bp}$ and $679 \mathrm{bp}$ are illustrated, while the lower panel shows promoter deletions in a regulatory section containing W-box elements between $-479 \mathrm{bp}$ and $-549 \mathrm{bp}$. Central sequences of the $P R-1$ promoter are highlighted by individual colors and bolt letters respectively. 


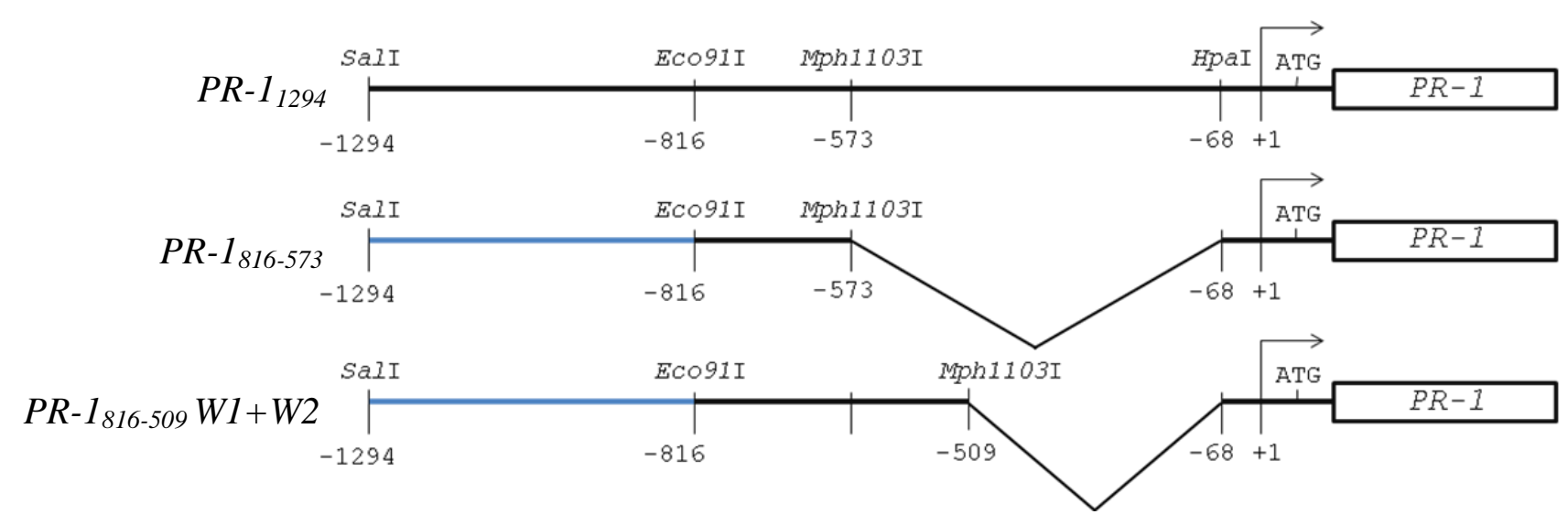

Figure 6.2: Design of $P R-1$ promoter deletion constructs.

The blue labeled areas illustrate 478bp substitutions against a stretch of coding sequence derived from the CAT gene, while the deletions in the downstream section of the $3^{`} D$ promoter constructs differ in respect to their size.

\subsection{Binary vectors used for transgenic- and transient reporter gene studies}

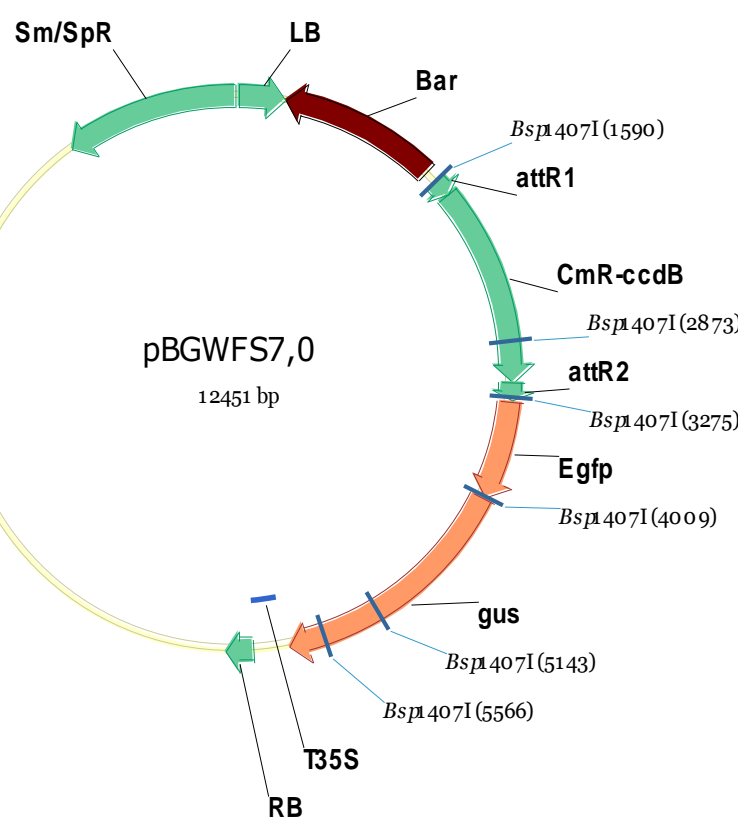

Figure 6.3: Plasmid map of the binary reporter gene (Egfp-GUS fusion) vector pBGWFS7 used for transient and stable transformations. 


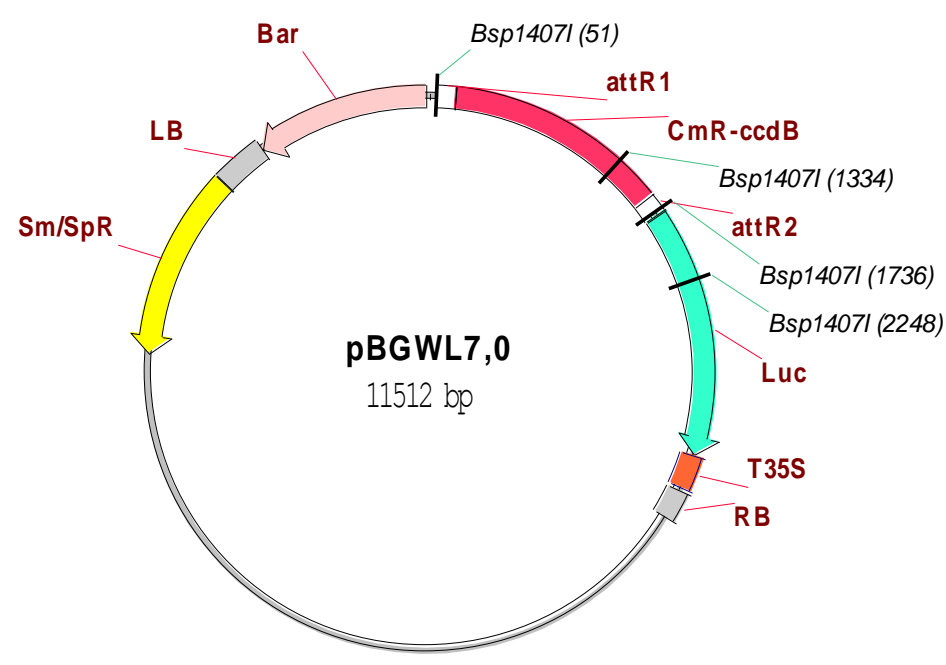

Figure 6.4: Plasmid map of the binary reporter gene vector (LUC) pBGWL7 used for $A$. tumefaciens mediated gene transfer.

\subsection{Overview of relative promoter activities}

\subsubsection{LS-constructs (pBGWL7)}

\begin{tabular}{|c|c|c|c|}
\hline $\begin{array}{l}\text { Promoter } \\
\text { derivative }\end{array}$ & plant & $\begin{array}{l}\text { Uninduced } \\
\text { expression }\end{array}$ & $\begin{array}{c}\text { Induced } \\
\text { expression }\end{array}$ \\
\hline \multirow{4}{*}{$P R-1_{1294}$} & $\mathrm{wt}$ & - & ++ \\
\hline & nprl-1 & - & - \\
\hline & snil-1 & + & +++ \\
\hline & snil-1 nprl-1 & + & ++ \\
\hline \multirow[t]{3}{*}{$L S 4_{\text {mut }}$} & wt & - & ++ \\
\hline & nprl-1 & - & - \\
\hline & snil-1 & + & +++ \\
\hline \multirow[t]{3}{*}{$L S 5_{\text {mut }}$} & $\mathrm{wt}$ & - & ++ \\
\hline & nprl-1 & - & - \\
\hline & snil-1 & + & ++ \\
\hline \multirow[t]{2}{*}{$L S 7_{\text {mut }}$} & $\mathrm{wt}$ & - & + \\
\hline & snil-1 & ++ & ++ \\
\hline$L S 10_{\text {mut }}$ & $\mathrm{wt}$ & - & + \\
\hline
\end{tabular}




\begin{tabular}{|l|l|c|c|}
\hline \multicolumn{1}{|c|}{$\begin{array}{c}\text { Promoter } \\
\text { derivative }\end{array}$} & plant & \multicolumn{1}{c|}{$\begin{array}{c}\text { Uninduced } \\
\text { expression }\end{array}$} & $\begin{array}{c}\text { Induced } \\
\text { expression }\end{array}$ \\
\hline$L S 4 / 5_{\mathrm{mut}}$ & wt & + & ++ \\
\hline & snil-1 & - & - \\
\hline$L S 4 / 7_{\mathrm{mut}}$ & wt & + & ++ \\
\hline & snil-1 & + & + \\
\hline$L S 5 / 7_{\mathrm{mut}}$ & wt & ++ & ++ \\
\hline & snil-1 & ++ & +++ \\
\hline$L S 7-L S 10_{\mathrm{mut}}$ & wt & - & - \\
\hline & snil-1 & ++ & ++ \\
\hline$L S 4 / 5 / 7_{\mathrm{mut}}$ & wt & - & - \\
\hline & snil-1 & + & + \\
\hline
\end{tabular}

\subsection{2 as-1 element constructs (pBGWL7)}

\begin{tabular}{|c|c|c|c|}
\hline $\begin{array}{l}\text { Promoter } \\
\text { derivative }\end{array}$ & plant & $\begin{array}{l}\text { Uninduced } \\
\text { expression }\end{array}$ & $\begin{array}{l}\text { Induced } \\
\text { expression }\end{array}$ \\
\hline \multirow[t]{3}{*}{$P R-1_{1294}$} & wt & - & ++ \\
\hline & nprl-1 & - & - \\
\hline & snil-1 & + & +++ \\
\hline \multirow[t]{3}{*}{$P R-1_{1294}$ as -1} & $\mathrm{wt}$ & ++++ & +++++ \\
\hline & snil-1 & ++++ & +++++ \\
\hline & nprl-1 & +++ & ++++ \\
\hline \multirow[t]{3}{*}{$P R-1_{816-573}$ as -1} & wt & +++ & +++ \\
\hline & snil-1 & +++ & +++ \\
\hline & nprl-1 & +++ & +++ \\
\hline PR-1 1294 as-1 LS10 & wt & +++ & ++++ \\
\hline \multirow[t]{2}{*}{ LS5aaaaLS7 } & wt & - & - \\
\hline & snil-1 & + & +++ \\
\hline
\end{tabular}




\subsection{3 as-1 element constructs (pBGWFS7)}

\begin{tabular}{|c|c|c|c|}
\hline $\begin{array}{l}\text { Promoter } \\
\text { derivative }\end{array}$ & plant & $\begin{array}{l}\text { Uninduced } \\
\text { expression }\end{array}$ & $\begin{array}{c}\text { Induced } \\
\text { expression }\end{array}$ \\
\hline \multirow[t]{2}{*}{$P R-1_{1294}$} & wt & - & ++ \\
\hline & nprl-1 & - & - \\
\hline \multirow[t]{2}{*}{$P R-1_{1294} a s-1$} & wt & ++ & +++ \\
\hline & nprl-1 & ++ & +++ \\
\hline \multirow[t]{2}{*}{$P R-1_{816-573} a s-1$} & wt & ++ & ++ \\
\hline & nprl-1 & ++ & ++ \\
\hline $\begin{array}{l}\text { PR-1 } 816-573 \quad \text { as }-1 \\
\text { linker }\end{array}$ & wt & - & - \\
\hline \multirow[t]{2}{*}{$P R-1_{1294}$ as-1linker } & wt & ++ & +++ \\
\hline & nprl-1 & ++ & +++ \\
\hline $\begin{array}{l}\text { PR-1 } 1294 \text { as }-1 P R-1 \\
\text { linker }\end{array}$ & wt & - & - \\
\hline \multirow{2}{*}{$\begin{array}{l}P R-1_{1294} \quad(\text { as }-1 / a s- \\
1 / P R 1)\end{array}$} & wt & ++ & +++ \\
\hline & nprl-1 & ++ & +++ \\
\hline $\begin{array}{l}P R-1_{1294}(P R-1 / P R- \\
1 / a s-1)\end{array}$ & wt & - & - \\
\hline
\end{tabular}




\subsubsection{W-box constructs (pBGWFS7)}

\begin{tabular}{|c|c|c|c|}
\hline $\begin{array}{l}\text { Promoter } \\
\text { derivative }\end{array}$ & plant & $\begin{array}{l}\text { Uninduced } \\
\text { expression }\end{array}$ & $\begin{array}{l}\text { Induced } \\
\text { expression }\end{array}$ \\
\hline$P R-1_{1294}$ & wt & - & ++ \\
\hline$\Delta W 1$ & wt & - & $(+)$ \\
\hline$\Delta W 1-2$ & wt & - & $(+)$ \\
\hline$\Delta W 1-3$ & wt & - & $(+)$ \\
\hline$\Delta W 2$ & wt & - & + \\
\hline $3^{\prime} \mathrm{D}+23 b p$ & wt & - & - \\
\hline $3 \mathrm{D}+\mathrm{W} 1$ & wt & - & + \\
\hline $\begin{array}{l}3 \mathrm{D}+\mathrm{W} 1+\mathrm{W} 2+ \\
\mathrm{W} 3\end{array}$ & wt & - & $+(+)$ \\
\hline
\end{tabular}

\subsubsection{W-box constructs (pBGWL7)}

\begin{tabular}{|l|l|l|l|}
\hline \multicolumn{2}{|c}{$\begin{array}{c}\text { Promoter } \\
\text { derivative }\end{array}$} & plant & \multicolumn{1}{c|}{$\begin{array}{c}\text { Uninduced } \\
\text { expression }\end{array}$} \\
\hline$P R-1_{1294}$ & wt & - & $\begin{array}{c}\text { Induced } \\
\text { expression }\end{array}$ \\
\hline & snil-1 & + & ++ \\
\hline$\Delta W 1$ & snil-1 & + & +++ \\
\hline$L S 4 / 5 \Delta W 1-3$ & snil-1 & + & ++ \\
\hline
\end{tabular}




\subsection{Overview of the LS-construct promoter activities in wt and snil-1}

The experiments presented above show a complex mode of regulation occurring on $P R$ 1 promoter level. For a better overview of the different promoter constructs in wt and snil-1 the relative LUC activities are compared in a single diagram. Figure $\mathbf{6 . 5}$ shows the expression levels of all introduced $L S$-constructs as well as the $P R-1_{816-573}$ promoter derivative in wt and snil-1.

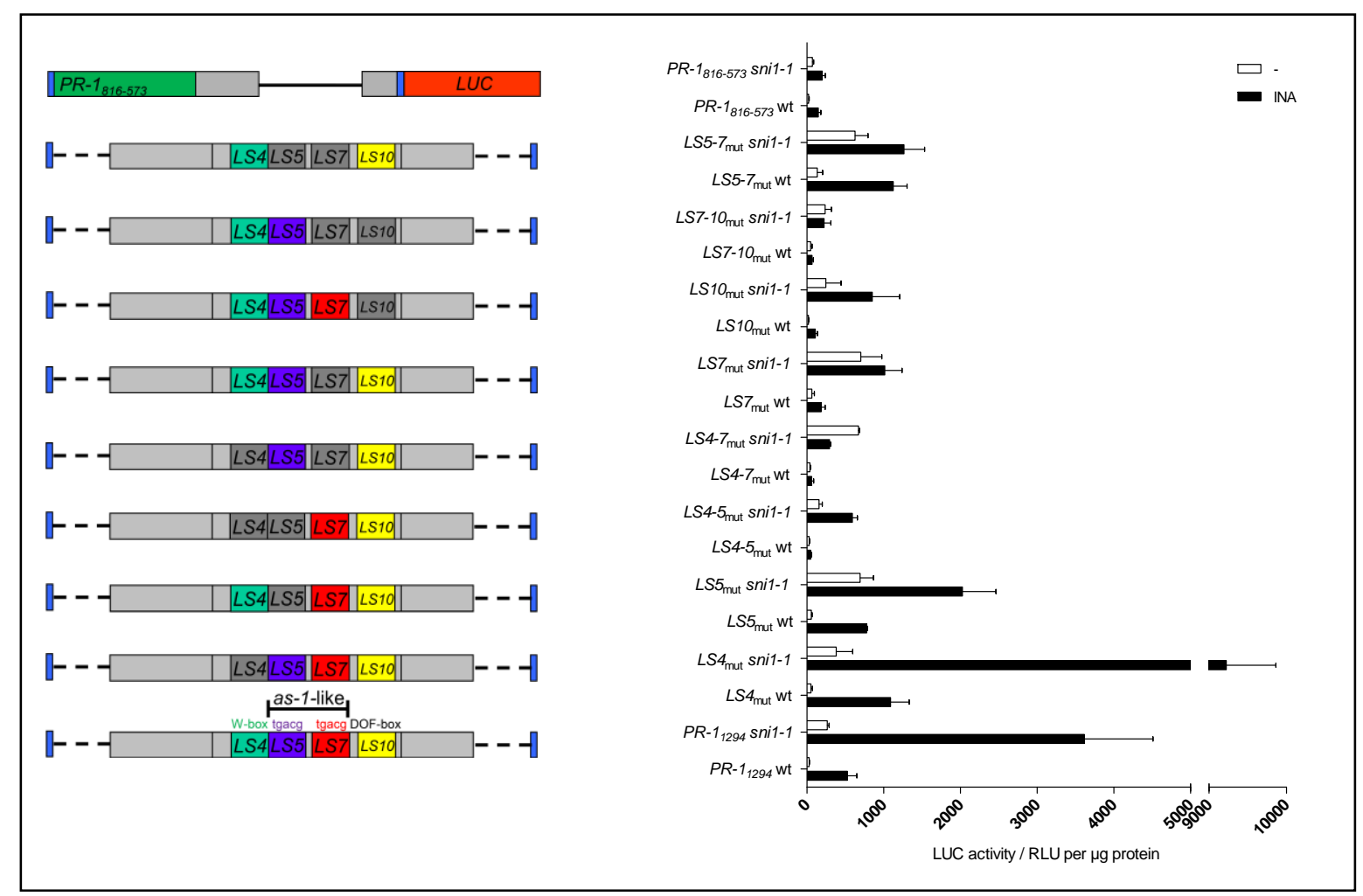

Figure 6.5: Overview of the different $L S$-promoter constructs in wt and sni1-1 mutant background. 


\subsection{Expression of WRKY46, WRKY53 and WRKY70 in wt and sni1-1 under inducing- and non-inducing conditions}

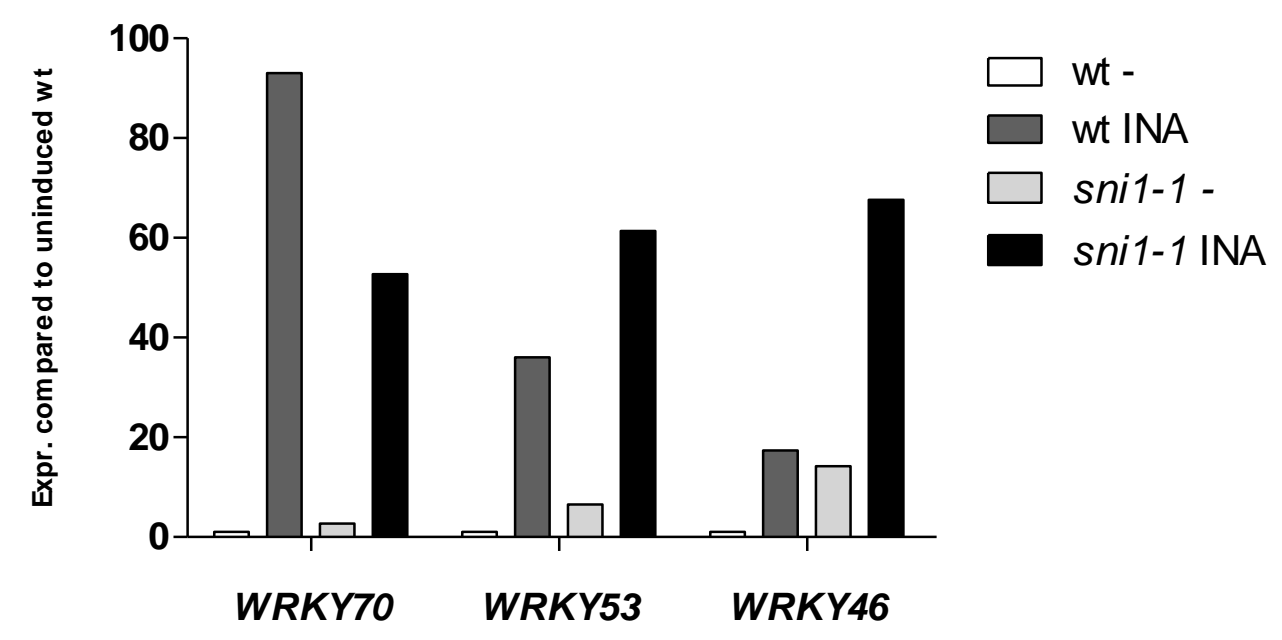

Figure 6.6: RT-PCR analysis of WRKY70, WRKY53 and WRKY46 expression in wildtype and sni1-1.

Plants were grown for 18 days on MS-plates supplemented with- or without $30 \mu \mathrm{M}$ INA. White columns show uninduced - whereas dark grey columns display induced expression levels in wt. The transcript levels in snil-1 are displayed by light grey (non-induced) and black columns (INA treated). Expression of WRKY46 is strongly influenced in snil-1 whereas the other two WRKY TFs only show weak (WRKY53) or no de-repression (WRKY70) in this mutant. 


\section{Bibliography}

Adie BA, Pérez-Pérez J, Pérez-Pérez MM, Godoy M, Sánchez-Serrano JJ, Schmelz EA and Solano R. 2007. ABA is an essential signal for plant resistance to pathogens affecting JA biosynthesis and the activation of defenses in Arabidopsis. The Plant Cell 19, no. 5 (May): 1665-81.

Alonso JM, Hirayama T, Roman G, Nourizadeh S and Ecker JR. 1999. EIN2, a bifunctional transducer of ethylene and stress responses in Arabidopsis. Science (New York, N.Y.) 284, no. 5423 (June 25): 2148-52.

Baranowskij N, Frohberg C, Prat S and Willmitzer L. 1994. A novel DNA binding protein with homology to Myb oncoproteins containing only one repeat can function as a transcriptional activator. The EMBO Journal 13, no. 22 (November 15): 5383-5392.

Bender CL, Alarcon-Chaidez F and Gross DC. 1999. Pseudomonas syringae Phytotoxins: Mode of Action, Regulation, and Biosynthesis by Peptide and Polyketide Synthetases. Microbiol. Mol. Biol. Rev. 63, no. 2 (June 1): 266-292.

Benfey PN, and Chua NH. 1990. The Cauliflower Mosaic Virus 35S Promoter: Combinatorial Regulation of Transcription in Plants. Science (New York, N.Y.) 250, no. 4983 (November 16): 959-966.

Bernard P, Kézdy KE, Van Melderen L, Steyaert J, Wyns L, Pato ML, Higgins PN and Couturier M. 1993. The F plasmid CcdB protein induces efficient ATP-dependent DNA cleavage by gyrase. Journal of Molecular Biology 234, no. 3 (December 5): 534-41.

Bi YM, Kenton P, Mur L, Darby R and J Draper. 1995. Hydrogen peroxide does not function downstream of salicylic acid in the induction of PR protein expression. The Plant Journal: For Cell and Molecular Biology 8, no. 2 (August): 235-45.

Blanco F, Garretón V, Frey N, Dominguez C, Pérez-Acle T, Van der Straeten D, Jordana $X$ and Holuigue L. 2005. Identification of NPR1-dependent and independent genes early induced by salicylic acid treatment in Arabidopsis. Plant Molecular Biology 59, no. 6 (December): 927-44.

Blanco F, Salinas P, Cecchini N, Jordana X, Van Hummelen P, Alvarez M and Loreto Holuigue. 2009. Early genomic responses to salicylic acid in Arabidopsis. Plant Molecular Biology (February 7):79-102. 
Cao H, Glazebrook J, Clarke JD, Volko S and Dong X. 1997. The Arabidopsis NPR1 gene that controls systemic acquired resistance encodes a novel protein containing ankyrin repeats. Cell 88, no. 1 (January 10): 57-63.

Cao H, Bowling SA, Gordon AS and Dong X. 1994. Characterization of an Arabidopsis Mutant That Is Nonresponsive to Inducers of Systemic Acquired Resistance. The Plant Cell 6, no. 11 (November): 1583-1592.

Chen W, Chao G and Singh KB. 1996. The promoter of a H2O2-inducible, Arabidopsis glutathione S-transferase gene contains closely linked OBF- and OBP1-binding sites. The Plant Journal: For Cell and Molecular Biology 10, no. 6 (December): 955-66.

Chen W and Singh KB. 1999. The auxin, hydrogen peroxide and salicylic acid induced expression of the Arabidopsis GST6 promoter is mediated in part by an ocs element. The Plant Journal: For Cell and Molecular Biology 19, no. 6 (September): 667-77.

Chen YF, Etheridge $\mathbf{N}$ and Schaller GE. 2005. Ethylene Signal Transduction. Ann Bot 95, no. 6 (May 1): 901-915.

Chen Z, Silva H, and Klessig DF. 1993. Active oxygen species in the induction of plant systemic acquired resistance by salicylic acid. Science (New York, N.Y.) 262, no. 5141 (December 17): 1883-6.

Chini A, Fonseca S, Fernández G, Adie B, Chico JM, Lorenzo O, García-Casado G, Lopez-Vidriero I, Lozano FM, Ponce MR, Micol JL and Solano R. 2007. The JAZ family of repressors is the missing link in jasmonate signalling. Nature 448, no. 7154 (August 9): 666-71.

Chisholm ST, Coaker G, Day B and Staskawicz BJ. 2006. Host-microbe interactions: shaping the evolution of the plant immune response. Cell 124, no. 4 (February 24): 803-14.

Chuang CF, Running MP, Williams RW, and Meyerowitz EM. 1999. The PERIANTHIA gene encodes a bZIP protein involved in the determination of floral organ number in Arabidopsis thaliana. Genes \& Development 13, no. 3 (February 1): 334-44.

Clough SJ. 2005. Floral dip: agrobacterium-mediated germ line transformation. Methods in Molecular Biology (Clifton, N.J.) 286: 91-102.

De Vos M, Van Oosten VR, Van Poecke MP, Van Pelt JA, Pozo MJ, Mueller MJ, Buchala AJ, Mêtraux JP, Van Loon LC, Dicke M and Pieterse. 2005. Signal signature and transcriptome changes of Arabidopsis during pathogen and insect attack. Molecular Plant-Microbe Interactions: MPMI 18, no. 9 (September): 923-37. 
Dean JV, Mohammed LA and Fitzpatrick T. 2005. The formation, vacuolar localization, and tonoplast transport of salicylic acid glucose conjugates in tobacco cell suspension cultures. Planta 221, no. 2 (May): 287-96.

Delaney TP, Friedrich L and Ryals JA. 1995. Arabidopsis signal transduction mutant defective in chemically and biologically induced disease resistance. Proceedings of the National Academy of Sciences of the United States of America 92, no. 14 (July 3): 6602-6.

Després C, DeLong C, Glaze S, Liu E and Fobert PR. 2000. The Arabidopsis NPR1/NIM1 protein enhances the DNA binding activity of a subgroup of the TGA family of bZIP transcription factors. The Plant Cell 12, no. 2 (February): 279-90.

Després C, Chubak C, Rochon A, Clark R, Bethune T, Desveaux D and Fobert PR. 2003. The Arabidopsis NPR1 disease resistance protein is a novel cofactor that confers redox regulation of DNA binding activity to the basic domain/leucine zipper transcription factor TGA1. The Plant Cell 15, no. 9 (September): 2181-91.

Dong J, Chen $\mathbf{C}$ and Chen Z. 2003. Expression profiles of the Arabidopsis WRKY gene superfamily during plant defense response. Plant Molecular Biology 51, no. 1 (January): 21-37.

Dong X. 2001. Genetic dissection of systemic acquired resistance. Current Opinion in Plant Biology 4, no. 4 (August): 309-14.

Dong X. 2004. NPR1, all things considered. Current Opinion in Plant Biology 7, no. 5 (October): 547-52.

Du H and Klessig DF. 1997. Identification of a Soluble, High-Affinity Salicylic AcidBinding Protein in Tobacco. Plant Physiology 113, no. 4 (April): 1319-1327.

Durner J and Klessig DF. 1995. Inhibition of ascorbate peroxidase by salicylic acid and 2,6-dichloroisonicotinic acid, two inducers of plant defense responses. Proceedings of the National Academy of Sciences of the United States of America 92, no. 24 (November 21): 11312-6.

Durrant WE and Dong X. 2004. Systemic acquired resistance. Annual Review of Phytopathology 42: 185-209.

Durrant WE, Wang S and Dong X. 2007. Arabidopsis SNI1 and RAD51D regulate both gene transcription and DNA recombination during the defense response. Proceedings of the National Academy of Sciences of the United States of America 104, no. 10 (March 6): 4223-7. 
Duval M, Hsieh TF, Kim SY and Thomas TL. 2002. Molecular characterization of AtNAM: a member of the Arabidopsis NAC domain superfamily. Plant Molecular Biology 50, no. 2 (September): 237-248.

Eulgem T. 2005. Regulation of the Arabidopsis defense transcriptome. Trends in Plant Science 10, no. 2 (February): 71-8.

Eulgem T and Somssich IE. 2007. Networks of WRKY transcription factors in defense signaling. Current Opinion in Plant Biology 10, no. 4 (August): 366-71.

Felix G, Duran JD, Volko S and Boller T. 1999. Plants have a sensitive perception system for the most conserved domain of bacterial flagellin. The Plant Journal: For Cell and Molecular Biology 18, no. 3 (May): 265-76.

Ferrari S, Galletti R, Denoux C, De Lorenzo G, Ausubel FM and Dewdney J. 2007. Resistance to Botrytis cinerea induced in Arabidopsis by elicitors is independent of salicylic acid, ethylene, or jasmonate signaling but requires PHYTOALEXIN DEFICIENT3. Plant Physiology 144, no. 1 (May): 367-79.

Feys BJ, Moisan LJ, Newman MA and Parker JE. 2001. Direct interaction between the Arabidopsis disease resistance signaling proteins, EDS1 and PAD4. The EMBO Journal 20, no. 19 (October 1): 5400-11.

Feys BJ and Parker JE. 2000. Interplay of signaling pathways in plant disease resistance. Trends in Genetics: TIG 16, no. 10 (October): 449-55.

Fode B, Siemsen T, Thurow C, Weigel R and Gatz C. 2008. The Arabidopsis GRAS Protein SCL14 Interacts with Class II TGA Transcription Factors and Is Essential for the Activation of Stress-Inducible Promoters. The Plant Cell 20, no. 11 (November): 3122-35.

Friedrich L, Lawton K, Ruess W, Masner P, Specker N, Rella MG, Meier B, Dincher S, Staub T, Uknes S, Mêtreaux JP, Kessmann H and Ryals J. 1996. A benzothiadiazole derivative induces systemic acquired resistance in tobacco. The Plant Journal 10, no. 1: 61-70.

Gaffney T, Friedrich L, Vernooij B, Negrotto D, Nye G, Uknes S, Ward E, Kessmann $H$ and Ryals J. 1993. Requirement of Salicylic Acid for the Induction of Systemic Acquired Resistance. Science (New York, N.Y.) 261, no. 5122 (August 6): 754-756.

Gao Z, Chen YF, Randlett MD, Zhao XC, Findell JL, Kieber JJ and Schaller GE. 2003. Localization of the Raf-like kinase CTR1 to the endoplasmic reticulum of Arabidopsis through participation in ethylene receptor signaling complexes. The Journal of Biological Chemistry 278, no. 36 (September 5): 34725-32. 
Glazebrook J. 2001. Genes controlling expression of defense responses in Arabidopsis-2001 status. Current Opinion in Plant Biology 4, no. 4 (August): 301-308.

Glazebrook J, Rogers EE and Ausubel FM. 1996. Isolation of Arabidopsis mutants with enhanced disease susceptibility by direct screening. Genetics 143 , no. 2 (June): 973-82.

Grant $M$ and Lamb C. 2006. Systemic immunity. Current Opinion in Plant Biology 9, no. 4 (August): 414-20.

Hanahan D. 1983. Studies on transformation of Escherichia coli with plasmids. Journal of Molecular Biology 166, no. 4 (June 5): 557-80.

Hartley JL, Temple GF and Brasch MA. 2000. DNA cloning using in vitro sitespecific recombination. Genome Research 10, no. 11 (November): 1788-95.

Heinekamp T, Kuhlmann M, Lenk A, Strathmann A and Dröge-Laser W. 2002. The tobacco bZIP transcription factor BZI-1 binds to G-box elements in the promoters of phenylpropanoid pathway genes in vitro, but it is not involved in their regulation in vivo. Molecular Genetics and Genomics: $M G G$ 267, no. 1 (March): 16-26.

Hepworth SR, Zhang Y, McKim S, Li X and Haughn GW. 2005. BLADE-ONPETIOLE-dependent signaling controls leaf and floral patterning in Arabidopsis. The Plant Cell 17, no. 5 (May): 1434-48.

Jakoby M, Weisshaar B, Dröge-Laser W, Vicente-Carbajosa J, Tiedemann J, Kroj T and Parcy F. 2002. bZIP transcription factors in Arabidopsis. Trends in Plant Science 7, no. 3 (March): 106-11.

Jalali BL, Bhargava S and Kamble A. 2006. Signal Transduction and Transcriptional Regulation of Plant Defence Responses. Journal of Phytopathology 154, no. 2: 65-74.

Johnson C, Boden E and Arias J. 2003. Salicylic acid and NPR1 induce the recruitment of trans-activating TGA factors to a defense gene promoter in Arabidopsis. The Plant Cell 15, no. 8 (August): 1846-58.

Kachroo P, Yoshioka K, Shah J, Dooner HK and Klessig DF. 2000. Resistance to turnip crinkle virus in Arabidopsis is regulated by two host genes and is salicylic acid dependent but NPR1, ethylene, and jasmonate independent. The Plant Cell 12, no. 5 (May): 677-90.

van Kan JA. 2006. Licensed to kill: the lifestyle of a necrotrophic plant pathogen. Trends in Plant Science 11, no. 5 (May): 247-53. 
Katagiri F, Lam E and Chua NH. 1989. Two tobacco DNA-binding proteins with homology to the nuclear factor CREB. Nature 340, no. 6236 (August 31): 727730 .

Katsir L, Schilmiller AL, Staswick PE, He SY and Howe GA. 2008. COI1 is a critical component of a receptor for jasmonate and the bacterial virulence factor coronatine. Proceedings of the National Academy of Sciences of the United States of America 105, no. 19 (May 13): 7100-5.

Kesarwani M, Yoo J and Dong X. 2007. Genetic Interactions of TGA Transcription Factors in the Regulation of Pathogenesis-Related Genes and Disease Resistance in Arabidopsis. Plant Physiology 144, no. 1 (May): 336-346.

Kessler A and Baldwin IT. 2002. Plant responses to insect herbivory: the emerging molecular analysis. Annual Review of Plant Biology 53: 299-328.

Kim HS and Delaney TP. 2002. Over-expression of TGA5, which encodes a bZIP transcription factor that interacts with NIM1/NPR1, confers SAR-independent resistance in Arabidopsis thaliana to Peronospora parasitica. The Plant Journal: For Cell and Molecular Biology 32, no. 2 (October): 151-63.

Kim KC, Lai Z, Fan B and Chen Z. 2008. Arabidopsis WRKY38 and WRKY62 Transcription Factors Interact with Histone Deacetylase 19 in Basal Defense. Plant Cell 20, no. 9 (September 1): 2357-2371.

Kirby J and Kavanagh TA. 2002. NAN fusions: a synthetic sialidase reporter gene as a sensitive and versatile partner for GUS. The Plant Journal: For Cell and Molecular Biology 32, no. 3 (November): 391-400.

Knoth C and Eulgem T. 2008. The oomycete response gene LURP1 is required for defense against Hyaloperonospora parasitica in Arabidopsis thaliana. The Plant Journal: For Cell and Molecular Biology 55, no. 1 (July): 53-64.

Krawczyk S, Thurow C, Niggeweg $R$ and Gatz C. 2002. Analysis of the spacing between the two palindromes of activation sequence- 1 with respect to binding to different TGA factors and transcriptional activation potential. Nucleic Acids Research 30, no. 3 (February 1): 775-81.

Kunkel BN and Brooks DM. 2002. Cross talk between signaling pathways in pathogen defense. Current Opinion in Plant Biology 5, no. 4 (August 1): 325-331.

Kunze G, Zipfel C, Robatzek S, Niehaus K, Boller T and Felix G. 2004. The N terminus of bacterial elongation factor Tu elicits innate immunity in Arabidopsis plants. The Plant Cell 16, no. 12 (December): 3496-507.

Lam E, Kato $\mathbf{N}$ and Lawton M. 2001. Programmed cell death, mitochondria and the plant hypersensitive response. Nature 411, no. 6839 (June 14): 848-53. 
Landschulz WH, Johnson PF, and McKnight SL. 1988. The leucine zipper: a hypothetical structure common to a new class of DNA binding proteins. Science (New York, N.Y.) 240, no. 4860 (June 24): 1759-64.

Landy A. 1989. Dynamic, structural, and regulatory aspects of lambda site-specific recombination. Annual Review of Biochemistry 58: 913-49.

Lawton KA, Potter SL, Uknes S and Ryals J. 1994. Acquired Resistance Signal Transduction in Arabidopsis Is Ethylene Independent. The Plant Cell 6, no. 5 (May): 581-588.

Lebel E, Heifetz P, Thorne L, Uknes S, Ryals J and Ward E. 1998. Functional analysis of regulatory sequences controlling PR-1 gene expression in Arabidopsis. The Plant Journal: For Cell and Molecular Biology 16, no. 2 (October): 223-33.

Lemaire SD. 2004. The glutaredoxin family in oxygenic photosynthetic organisms. Photosynthesis Research 79, no. 3: 305-18.

Leon J, Lawton MA and Raskin I. 1995. Hydrogen Peroxide Stimulates Salicylic Acid Biosynthesis in Tobacco. Plant Physiology 108, no. 4 (August): 16731678.

Leon-Reyes A, Spoel SH, De Lange ES, Abe H, Kobayashi M, Tsuda S, Millenaar FF, Welschen RAM, Ritsema T and Pieterse CMJ. 2009. Ethylene Modulates the Role of NPR1 in Cross-Talk Between Salicylate and Jasmonate Signaling. Plant Physiology (January 28).

Li J , Brader G and Palva ET. 2004. The WRKY70 transcription factor: a node of convergence for jasmonate-mediated and salicylate-mediated signals in plant defense. The Plant Cell 16, no. 2 (February): 319-331.

Li X, Zhang Y, Clarke JD, Li Y, and Dong X. 1999. Identification and cloning of a negative regulator of systemic acquired resistance, SNI1, through a screen for suppressors of npr1-1. Cell 98, no. 3 (August 6): 329-39.

Lipka U, Fuchs $R$ and Lipka V. 2008. Arabidopsis non-host resistance to powdery mildews. Current Opinion in Plant Biology 11, no. 4 (August): 404-411.

Lippok B, Birkenbihl RP, Rivory G, Brümmer J, Schmelzer E, Logemann E and Somssich IE. 2007. Expression of AtWRKY33 encoding a pathogen- or PAMPresponsive WRKY transcription factor is regulated by a composite DNA motif containing W box elements. Molecular Plant-Microbe Interactions: MPMI 20, no. 4 (April): 420-429.

Liu X and Lam E. 1994. Two binding sites for the plant transcription factor ASF-1 can respond to auxin treatments in transgenic tobacco. The Journal of Biological Chemistry 269, no. 1 (January 7): 668-75. 
Malamy J, Carr JP, Klessig DF and Raskin I. 1990. Salicylic Acid: A Likely Endogenous Signal in the Resistance Response of Tobacco to Viral Infection. Science (New York, N.Y.) 250, no. 4983 (November 16): 1002-1004.

Maldonado AM, Doerner P, Dixon RA, Lamb CJ and Cameron RK. 2002. A putative lipid transfer protein involved in systemic resistance signalling in Arabidopsis. Nature 419, no. 6905 (September 26): 399-403.

Maleck K, Levine A, Eulgem T, Morgan A, Schmid J, Lawton KA, Dangl JL and Dietrich RA. 2000. The transcriptome of Arabidopsis thaliana during systemic acquired resistance. Nature Genetics 26, no. 4 (December): 403-10.

Métraux JP, Signer H, Ryals J, Ward E, Wyss-Benz M, Gaudin J, Raschdorf K, Schmid E, Blum W and Inverardi B. 1990. Increase in Salicylic Acid at the Onset of Systemic Acquired Resistance in Cucumber. Science (New York, N.Y.) 250, no. 4983 (November 16): 1004-1006.

Meyer D, Pajonk S, Micali C, O'Connell R and Schulze-Lefert P. 2009. Extracellular transport and integration of plant secretory proteins into pathogeninduced cell wall compartments. The Plant Journal: For Cell and Molecular Biology 57, no. 6 (March): 986-99.

Mishina TA and Zeier J. 2007. Pathogen-associated molecular pattern recognition rather than development of tissue necrosis contributes to bacterial induction of systemic acquired resistance in Arabidopsis. The Plant Journal 50, no. 3: 500513.

Mosher RA, Durrant WE, Wang D, Song J, and Dong X. 2006. A comprehensive structure-function analysis of Arabidopsis SNI1 defines essential regions and transcriptional repressor activity. The Plant Cell 18, no. 7 (July): 1750-65.

Mou Z, Fan W and Dong X. 2003. Inducers of plant systemic acquired resistance regulate NPR1 function through redox changes. Cell 113, no. 7 (June 27): 93544.

Mueller S, Hilbert B, Dueckershoff K, Roitsch T, Krischke M, Mueller MJ and Berger S. 2008. General detoxification and stress responses are mediated by oxidized lipids through TGA transcription factors in Arabidopsis. The Plant Cell 20, no. 3 (March): 768-85.

Murray SL, Ingle RA, Petersen LN and Denby KJ. 2007. Basal resistance against Pseudomonas syringae in Arabidopsis involves WRKY53 and a protein with homology to a nematode resistance protein. Molecular Plant-Microbe Interactions: MPMI 20, no. 11 (November): 1431-8.

Mysore KS and Ryu CM. 2004. Nonhost resistance: how much do we know? Trends in Plant Science 9, no. 2 (February): 97-104. 
Ndamukong I, Abdallat AA, Thurow C, Fode B, Zander M, Weigel R and Gatz C. 2007. SA-inducible Arabidopsis glutaredoxin interacts with TGA factors and suppresses JA-responsive PDF1.2 transcription. The Plant Journal: For Cell and Molecular Biology 50, no. 1 (April): 128-39.

Neuenschwander U, Vernooij B, Friedrich L, Uknes S, Kessmann H and Ryals J. 1995. Is hydrogen peroxide a second messenger of salicylic acid in systemic acquired resistance? The Plant Journal 8, no. 2: 227-233.

Nomura K, Melotto M and He SY. 2005. Suppression of host defense in compatible plant-Pseudomonas syringae interactions. Current Opinion in Plant Biology 8, no. 4 (August): 361-368.

Park, SW, Kaimoyo E, Kumar D, Mosher S and DF Klessig. 2007. Methyl salicylate is a critical mobile signal for plant systemic acquired resistance. Science (New York, N.Y.) 318, no. 5847 (October 5): 113-6.

Pieterse CM and van Loon LC. 1999. Salicylic acid-independent plant defence pathways. Trends in Plant Science 4, no. 2 (February): 52-58.

Pieterse CM, van Wees SC, van Pelt JA, Knoester M, Laan R, Gerrits H, Weisbeek PJ and van Loon LC. 1998. A novel signaling pathway controlling induced systemic resistance in Arabidopsis. The Plant Cell 10, no. 9 (September): 157180 .

Qin XF, Holuigue L, Horvath DM and Chua NH. 1994. Immediate early transcription activation by salicylic acid via the cauliflower mosaic virus as-1 element. The Plant Cell 6, no. 6 (June): 863-74.

Qu LJ, and Zhu YX. 2006. Transcription factor families in Arabidopsis: major progress and outstanding issues for future research. Current Opinion in Plant Biology 9, no. 5 (October): 544-549.

Redman J, Whitcraft J, Johnson $\mathbf{C}$ and Arias J. 2002. Abiotic and biotic stress differentially stimulate as- 1 element activity in Arabidopsis. Plant Cell Reports 21, no. 2: 180-185.

Reymond P, Bodenhausen N, Van Poecke RM, Krishnamurthy V, Dicke,M and Farmer EE. 2004. A conserved transcript pattern in response to a specialist and a generalist herbivore. The Plant Cell 16, no. 11 (November): 3132-47.

Rochon A, Boyle P, Wignes T, Fobert PR and Després C. 2006. The coactivator function of Arabidopsis NPR1 requires the core of its BTB/POZ domain and the oxidation of C-terminal cysteines. The Plant Cell 18, no. 12 (December): 367085 . 
Rushton PJ , Macdonald H, Huttly AK, Lazarus CM and Hooley R. 1995. Members of a new family of DNA-binding proteins bind to a conserved ciselement in the promoters of alpha-Amy2 genes. Plant Molecular Biology 29, no. 4 (November): 691-702.

Ryals J, Lawton KA, Delaney TP, Friedrich L, Kessmann H, Neuenschwander U, Uknes S, Vernooij B and Weymann K. 1995. Signal transduction in systemic acquired resistance. Proceedings of the National Academy of Sciences of the United States of America 92, no. 10 (May 9): 4202-5.

Ryals J, Weymann K, Lawton K, Friedrich L, Ellis D, Steiner HY, Johnson J, Delaney TP, Jesse T, Vos $P$ and Uknes S. 1997. The Arabidopsis NIM1 protein shows homology to the mammalian transcription factor inhibitor I kappa B. The Plant Cell 9, no. 3 (March): 425-39.

Ryals, JA, Neuenschwander UH, Willits MG, Molina A, Steiner HY and Hunt MD. 1996. Systemic Acquired Resistance. The Plant Cell 8, no. 10 (October): 18091819.

Yang SF and Hoffman NE. 2003. Ethylene Biosynthesis and its Regulation in Higher Plants. Annual reviews of plant physiology no. 35 (November 28): 155-89.

Schafer FQ and Buettner GR. 2001. Redox environment of the cell as viewed through the redox state of the glutathione disulfide/glutathione couple. Free Radical Biology \& Medicine 30, no. 11 (June 1): 1191-212.

Sheen J. 2001. Signal transduction in maize and Arabidopsis mesophyll protoplasts. Plant Physiology 127, no. 4 (December): 1466-75.

Shirasu K, Nakajima H, Rajasekhar VK, Dixon RA and Lamb C. 1997. Salicylic acid potentiates an agonist-dependent gain control that amplifies pathogen signals in the activation of defense mechanisms. The Plant Cell 9, no. 2 (February): 261-70.

Song YH, Song NY, Shin SY, Kim HJ, Yun DJ, Lim CO, Lee SY, Kang KY and Hong JC. 2008. Isolation of CONSTANS as a TGA4/OBF4 interacting protein. Molecules and Cells 25, no. 4 (June 30): 559-65.

Spoel SH, Johnson JS and Dong X. 2007. Regulation of tradeoffs between plant defenses against pathogens with different lifestyles. Proceedings of the National Academy of Sciences of the United States of America 104, no. 47 (November 20): 18842-7.

Spoel SH, Koornneef A, Claessens SM, Korzelius JP, Van Pelt JA, Mueller MJ, Buchala AJ, Mêtreaux JP, Brown R, Kazan K, Van Loon LC, Dong X and Pieterse CM. 2003. NPR1 modulates cross-talk between salicylate- and jasmonate-dependent defense pathways through a novel function in the cytosol. The Plant Cell 15, no. 3 (March): 760-70. 
Stahl EA and Bishop JG. 2000. Plant-pathogen arms races at the molecular level. Current Opinion in Plant Biology 3, no. 4 (August): 299-304.

Staskawicz BJ, Ausubel FM, Baker BJ, Ellis JG and Jones JD. 1995. Molecular genetics of plant disease resistance. Science (New York, N.Y.) 268, no. 5211 (May 5): 661-7.

Staswick PE. 2008. JAZing up jasmonate signaling. Trends in Plant Science 13, no. 2 (February): 66-71.

Stintzi A, Weber H, Reymond P, Browse J and Farmer EE. 2001. Plant defense in the absence of jasmonic acid: the role of cyclopentenones. Proceedings of the National Academy of Sciences of the United States of America 98, no. 22 (October 23): 12837-42.

Strawn MA, Marr SK, Inoue K, Inada N, Zubieta C and Wildermuth MC. 2007. Arabidopsis isochorismate synthase functional in pathogen-induced salicylate biosynthesis exhibits properties consistent with a role in diverse stress responses. The Journal of Biological Chemistry 282, no. 8 (February 23): 5919-33.

Strompen G, Grüner R and Pfitzner UM. 1998. An as-1-like motif controls the level of expression of the gene for the pathogenesis-related protein 1a from tobacco. Plant Molecular Biology 37, no. 5 (July): 871-83.

Takahashi H, Miller J, Nozaki Y, Takeda M, Shah J, Hase S, Ikegami M, Ehara Y and Dinesh-Kumar SP. 2002. RCY1, an Arabidopsis thaliana RPP8/HRT family resistance gene, conferring resistance to cucumber mosaic virus requires salicylic acid, ethylene and a novel signal transduction mechanism. The Plant Journal: For Cell and Molecular Biology 32, no. 5 (December): 655-67.

Thines B, Katsir L, Melotto M, Niu Y, Mandaokar A, Liu G, Nomura K, He SY, Howe GA and Browse J. 2007. JAZ repressor proteins are targets of the SCF(COI1) complex during jasmonate signalling. Nature 448, no. 7154 (August 9): 661-5.

Thomma BP, Eggermont K, Penninckx IA, Mauch-Mani B, Vogelsang R, Cammue BP and Broekaert WF. 1998. Separate jasmonate-dependent and salicylatedependent defense-response pathways in Arabidopsis are essential for resistance to distinct microbial pathogens. Proceedings of the National Academy of Sciences of the United States of America 95, no. 25 (December 8): 15107-11.

Thomma BP, Eggermont K, Tierens KF and Broekaert WF. 1999. Requirement of Functional Ethylene-Insensitive 2 Gene for Efficient Resistance of Arabidopsis to Infection by Botrytis cinerea. Plant Physiol. 121, no. 4 (December 1): 10931101. 
Thomma BP, Penninckx IA, Cammue BP and Broekaert WF. 2001. The complexity of disease signaling in Arabidopsis. Current Opinion in Immunology 13, no. 1 (February): 63-68.

Thurow C, Schiermeyer A, Krawczyk S, Butterbrodt T, Nickolov K and Gatz C. 2005. Tobacco bZIP transcription factor TGA 2.2 and related factor TGA2.1 have distinct roles in plant defense responses and plant development. The Plant Journal: For Cell and Molecular Biology 44, no. 1 (October): 100-113.

Truman W, de Zabala MT and Grant M. 2006. Type III effectors orchestrate a complex interplay between transcriptional networks to modify basal defence responses during pathogenesis and resistance. The Plant Journal: For Cell and Molecular Biology 46, no. 1 (April): 14-33.

Tsuda K, Sato M, Glazebrook J, Cohen JD and Katagiri F. 2008. Interplay between MAMP-triggered and SA-mediated defense responses. The Plant Journal: For Cell and Molecular Biology 53, no. 5 (March): 763-75.

Uknes S, Mauch-Mani B, Moyer M, Potter S, Williams S, Dincher S, Chandler D, Slusarenko A, Ward E and Ryals J. 1992. Acquired resistance in Arabidopsis. The Plant Cell 4, no. 6 (June): 645-56.

Uppalapati SR, Ayoubi P, Weng H, Palmer DA, Mitchell RE, Jones W and Bender CL. 2005. The phytotoxin coronatine and methyl jasmonate impact multiple phytohormone pathways in tomato. The Plant Journal: For Cell and Molecular Biology 42, no. 2 (April): 201-17.

Uquillas C, Letelier I, Blanco F, Jordana $X$ and Holuigue L. 2004. NPR1independent activation of immediate early salicylic acid-responsive genes in Arabidopsis. Molecular Plant-Microbe Interactions: MPMI 17, no. 1 (January): 34-42.

Van Loon LC. 1997. Induced resistance in plants and the role of pathogenesis-related proteins. European Journal of Plant Pathology 103, no. 9 (December 1): 753765.

Vernooij B, Friedrich L, Morse A, Reist R, Kolditz-Jawhar R, Ward E, Uknes S, Kessmann H and Ryals J. 1994. Salicylic Acid Is Not the Translocated Signal Responsible for Inducing Systemic Acquired Resistance but Is Required in Signal Transduction. The Plant Cell 6, no. 7 (July): 959-965.

Wang D, Amornsiripanitch $\mathbf{N}$ and Dong X. 2006. A genomic approach to identify regulatory nodes in the transcriptional network of systemic acquired resistance in plants. PLoS Pathogens 2, no. 11 (November): e123. 
Wang D, Pajerowska-Mukhtar K, Culler AH and Dong X. 2007. Salicylic acid inhibits pathogen growth in plants through repression of the auxin signaling pathway. Current Biology: CB 17, no. 20 (October 23): 1784-90.

Wang D, Weaver ND, Kesarwani $M$ and Dong X. 2005. Induction of protein secretory pathway is required for systemic acquired resistance. Science (New York, N.Y.) 308, no. 5724 (May 13): 1036-40.

Weigel RR, Bäuscher C, Pfitzner AJ and UM Pfitzner. 2001. NIMIN-1, NIMIN-2 and NIMIN-3, members of a novel family of proteins from Arabidopsis that interact with NPR1/NIM1, a key regulator of systemic acquired resistance in plants. Plant Molecular Biology 46, no. 2 (May): 143-60.

Weigel RR, Pfitzner UM and Gatz C. 2005. Interaction of NIMIN1 with NPR1 modulates PR gene expression in Arabidopsis. The Plant Cell 17, no. 4 (April): 1279-91.

Wildermuth MC, Dewdney J, Wu G and Ausubel FM. 2001. Isochorismate synthase is required to synthesize salicylic acid for plant defence. Nature 414, no. 6863 (November 29): 562-5.

Yanhui C, Xiaoyuan Y, Kun H, Meihua L, Jigang L, Zhaofeng G, Zhiqiang L, Yunfei Z, Xiaoxiao W, Xiaoming Q, Yunping S, Li Z, Xiaohui D, Jingchu L, Xing-Wang D, Zhangliang $C$, Hongya $G$ and Li-Jia $Q$. 2006. The MYB transcription factor superfamily of Arabidopsis: expression analysis and phylogenetic comparison with the rice MYB family. Plant Molecular Biology 60, no. 1 (January): 107-124.

Yu D, Chen C and Chen Z. 2001. Evidence for an important role of WRKY DNA binding proteins in the regulation of NPR1 gene expression. The Plant Cell 13, no. 7 (July): 1527-40.

Zhang Y, Tessaro MJ, Lassner M and Li X. 2003. Knockout analysis of Arabidopsis transcription factors TGA2, TGA5, and TGA6 reveals their redundant and essential roles in systemic acquired resistance. The Plant Cell 15, no. 11 (November): 2647-53.

Zhou JM, Trifa Y, Silva H, Pontier D, Lam E, Shah J and Klessig DF. 2000. NPR1 differentially interacts with members of the TGA/OBF family of transcription factors that bind an element of the PR-1 gene required for induction by salicylic acid. Molecular Plant-Microbe Interactions: MPMI 13, no. 2 (February): 191202. 


\section{Acknowledgement}

An erster Stelle möchte ich mich natürlich bei Frau Prof. Dr. Christiane Gatz für die Möglichkeit bedanken, in ihrer Arbeitsgruppe meine Promotion absolvieren zu dürfen. Desweiteren danke ich ihr für ihre sehr gute Betreuung und stetige Diskussionsbereitschaft.

Weiterer Dank gilt PD Dr. Wolfgang Dröge-Laser, der sich bereitwillig als Korreferent für dieses komplexe Werk zur Verfügung gestellt hat. Ich hoffe das Lesen hat dir halbwegs Freude bereitet.

Besonders möchte ich Sabine Piontek dafür danken, dass sie immer für mich da ist und mich in den vergangenen Jahren stets beim Projekt „Doktorarbeit“ unterstützt hat. Danke, mein Schatz!

Kommen wir nun zu meinen lieben Arbeitskollegen. Dr. Corinna Thurow gilt spezieller Dank, da sie mein Projekt initiiert hat und immer mit Rat und Tat zur Seite stand, v.a. wenn seltsame Banden auf Gelen auftauchten oder andere (auf den ersten Blick) unerklärliche Phänomene auftraten. Weiter danken möchte ich allen ehemaligen- und aktuellen Doktoranden und Diplomanden in der Arbeitsgruppe, natürlich besonders meinen langjährigen Begleiter/innen Katja Rindermann, Dr. Benjamin „Schilt“ Fode, Mark „Biff“ Zander, Julia Köster, Martin „Potti“ Muthreich und Simon „Shifty“ von Berlepsch. Für die kurzweilige Zeit innerhalb- und außerhalb des Labors danke ich euch sehr, an einige Geschichten werden wir uns wohl noch lange erinnern, ich gehe aus Platzmangel aber besser nicht darauf ein :). Schließlich möchte ich noch Dr. Guido Kriete für seine Hilfsbereitschaft bezüglich Computer- und Netzwerkproblemen danken.

Ganz besonderer Dank gilt unseren technischen Assistenten Larissa Kuntz, Anna Hermann und Ronald Scholz. Vor allem Larissa und Anna bin ich zu tiefsten Dank verpflichtet da ich ohne ihre Hilfe niemals einen so großen Datensatz hätte generieren können.

Spezieller Dank gilt weiterhin Silja Neizel für die Anfertigung ihrer Staatsexamensarbeit unter meiner Obhut, sowie der Armada an Praktikanten (unter ihnen v.a. Charlotte Roth) die mein Projekt mal mehr und mal überhaupt nicht voran gebracht hat.

Meiner Familie und meinen Freunden möchte ich natürlich auch an dieser Stelle für die Unterstützung während meiner Zeit als Doktorand danken.

Zum Schluss möchte ich noch der „Göttinger series of poker (GöSP)“, einer Organisation mittelprächtiger Pokerspieler die einige sehr lustige Veranstaltungen ausgetragen haben, meinen Dank aussprechen. Euer Geld war mir stets eine willkommene Finanzspritze. 


\section{Curriculum Vitae}

\section{Persönliches}

Name

Geburtsdatum/-ort

Staatsangehörigkeit

Deutsch

\section{Schule}

84-88

88-97

07/97-08/98

09/98-02/99

04/99-05/05

seit $09 / 05$

Göttingen, den 26. Mai 2009
Städt. Kath. Grundschule Brakel

Gymnasium Brede in Brakel, Allgemeine Hochschulreife

\section{Zivildienst}

Altenpflege und technischer Dienst im Altenheim St.Josefshaus Bökendorf

\section{Studium}

Universität-Gesamthochschule-Paderborn, Studiengang Technischer Umweltschutz

Johannes Gutenberg-Universität Mainz, Diplomstudiengang Biologie

Beginn der Promotion an der Georg-August-Universität Göttingen in der Arbeitsgruppe von Prof. Dr. Christiane Gatz. Dissertationsthema: „Functional Analysis of the Salicylic Acid-Responsive PR-1 Promoter in Arabidopsis thaliana" 